Access to Unnatural $\alpha$-Amino Acids via Visible-Light Mediated Decarboxylative Conjugate Addition to Dehydroalanine

Akshay A. Shah*†, Michael J. Kelly IIIt, James J. Perkins†

Department of Discovery Chemistry, Merck \& Co., Inc. 770 Sumneytown Pike, West Point, Pennsylvania 19486, USA

tequal contribution

Table of Contents $\quad$ Page

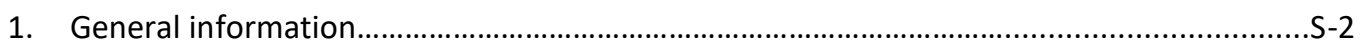

2. Experimental procedures and characterization data.........................................................S -3

A. Optimizations/Screening and synthesis of $\alpha$-amino benzyl esters 9 (Table 1)..............S-3

B. PMC synthesis of $\alpha$-amino benzyl esters $6 a-6 m$ (Table 2) ............................................

C. Batch synthesis of $\alpha$-amino benzyl esters $6 \mathbf{d}, \mathbf{6 n}-\mathbf{6 t}$ (Table 3).......................................S-12

D. Synthesis of 6 a on $1 \mathrm{mmol}$ scale via batch conditions i.e. method B..........................S-22

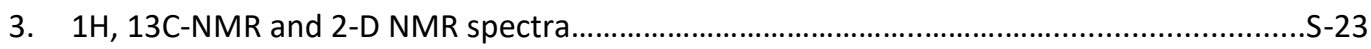




\section{General Information:}

Unless otherwise noted, all reactions were performed in capped vials (1-dram or 2-dram or scintillation vial). Nonaqueous reagents were transferred using syringe techniques. N,N-dimethylformamide (DMF), dichloroethane (DCE), dichloromethane $\left(\mathrm{CH}_{2} \mathrm{Cl}_{2}\right)$, dimethoxyethane (DME), acetonitrile (MeCN), methanol (MeOH) and ethyl acetate (EtOAc) were obtained in sealed, anhydrous bottles from Aldrich or Acros Organics. Bulk solvents used for reverse phase chromatographic purifications (0.1\% TFA in MeCN and $0.1 \%$ TFA in water) were acquired in $4 \mathrm{~L}$ bottles from Fisher and used without further treatment. Commercial reagents were obtained at the highest commercial quality and used without further purification, unless otherwise stated. Bis(tert-butaloxycarbonyl) dehydroalanine benzyl ester 5 was sourced from Enamine (EN300-183218; cas no. 1798715-69-1). Yields refer to chromatographically and spectroscopically (1H-NMR) homogeneous materials, unless otherwise stated. Reactions were monitored by LC-MS techniques using Waters Acquity UPLC, water/MeCN gradient and $0.1 \% \mathrm{v} / \mathrm{v}$ TFA as modifier. Chromatographic separations were performed using ISCO ACCQPrep HP125 or Gilson PLC 2020 unit with Waters SunFire C18 OBD 5 $\mu \mathrm{m} 30 \times 150 \mathrm{~mm}$ prep column. Nuclear magnetic resonance (NMR) spectra were recorded on a Bruker Avance NEO $500 \mathrm{MHz} N \mathrm{NMR}$ spectrometer and chemical shifts are reported relative to internal tetramethylsilane [0.00 ppm] for $1 \mathrm{H}$ NMR and residual undeuterated solvent for ${ }^{13} \mathrm{C}$ NMR [ $\delta 49.00$ (MeOD) or 39.52 (DMSO- $\left.\mathrm{d}_{6}\right)$ or $118.26\left(C D_{3} \mathrm{CN}\right)$ ppm]. The following abbreviations were used to explain the multiplicities: $s=\operatorname{singlet;} d=d o u b l e t ; t=t r i p l e t ; ~ q=$ quartet; $d d$ = doublet of doublet; $q d$ = quartet of doublet; $d d d$ = doublet of doublet of doublet; dtd = doublet of triplet of doublet; $\mathrm{m}=$ multiplet or nonequivalent resonances. NMR coupling constants are reported as $\mathrm{J}$ values in $\mathrm{Hz}$ and $\delta$ values in parts per million (ppm). High resolution mass measurements (HRMS) were obtained on Waters Synapt G1 QTOF instrument (ESI+ mode, source temperature: $130^{\circ} \mathrm{C}$ ) connected to Waters Acquity UPLC (column: Acquity UPLC BEH C18 1.7um $1.0 \times 50 \mathrm{~mm})$. 


\section{Experimental procedures and characterization data:}

A. Optimizations/Screening and synthesis of $\alpha$-amino benzyl ester 9

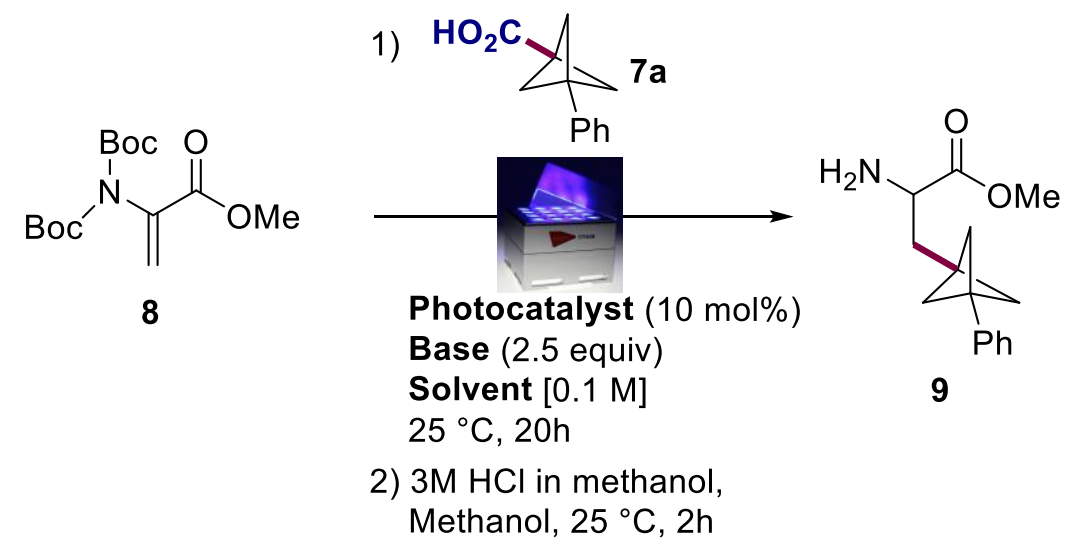

General procedure for screening (Table 1):

In a 2-dram vial were added bis-(tertbutaloxycarbonyl) dehydroalanine methyl ester (8) $(50 \mathrm{mg}, 0.166 \mathrm{mmol}, 1.0$ equiv), 3-phenylbicyclo[1.1.1]pentane-1-carboxylic acid (7a) $(47 \mathrm{mg}, 0.249 \mathrm{mmol}, 1.5$ equiv), and the appropriate photocatalyst $(0.017 \mathrm{mmol}, 0.1$ equiv). Solvent(s) was added to the vials $(1650 \mu \mathrm{L})$ followed by appropriate base ( $0.498 \mathrm{mmol}, 3.0$ equiv). Vials were sealed and then set on the Aldrich Syn LED parallel photoreactor ${ }^{\circledR}$ (wavelength: $465-470 \mathrm{~nm}$ ) overnight. After $20 \mathrm{~h}$ reaction time, DMSO $(1 \mathrm{~mL})$ was added to the mixture, which was filtered and the filtrate purified by reverse phase (C-18) HPLC $\left(30 \times 150 \mathrm{~mm}\right.$, Waters SunFire ${ }^{\circledR}$ OBD $\left.^{\mathrm{TM}} 10 \mathrm{mM}\right)$ eluting with a gradient of 5-95\% Acetonitrile/Water $+0.1 \%$ TFA over 15 minutes at $43 \mathrm{ml} / \mathrm{min}$. The fractions that contained product were combined and concentrated to provide crude product $9.1 \mathrm{ml}$ of $3 \mathrm{M} \mathrm{HCl} \mathrm{MeOH}$ was then added and the solution was stirred for 16 hours. After the reaction was complete, volatiles were removed in vacuo to provide methyl 2-amino-3-(3-phenylbicyclo[1.1.1]pentan-1-yl)propanoate hydrochloride 9 as a white solid.

Characterization data for $\mathbf{9}$ :

${ }^{1} \mathrm{H}$ NMR $\left(500 \mathrm{MHz}, \mathrm{DMSO}-\mathrm{d}_{6}\right) \delta 8.49(\mathrm{~s}, 3 \mathrm{H}), 7.30(\mathrm{t}, J=7.4 \mathrm{~Hz}, 2 \mathrm{H}), 7.25-7.14(\mathrm{~m}, 3 \mathrm{H}), 4.04(\mathrm{dd}, J=7.2,5.2 \mathrm{~Hz}, 1 \mathrm{H})$, $3.78(\mathrm{~s}, 3 \mathrm{H}), 2.28-2.01(\mathrm{~m}, 2 \mathrm{H}), 1.95(\mathrm{~s}, 6 \mathrm{H})$;

${ }^{13} \mathrm{C}$ NMR (126 MHz, MeOD) $\delta$ 161.6, 132.1, 119.7, 118.1, 117.4, 44.2, 44.1, 43.1, 33.9, 26.9, 24.2;

HRMS calcd for $\mathrm{C}_{15} \mathrm{H}_{20} \mathrm{NO}_{2}[\mathrm{M}+\mathrm{H}]^{+} 246.1489$ found 246.1479 


\section{B. PMC synthesis of $\alpha$-amino benzyl esters $6 a-6 \mathrm{~m}$ (Table 2 )}

Method A: General procedure for PMC synthesis

STEP 1: Stock Solution Preparation: A $50 \mathrm{~mL}$ flask was charged with bis-(tertbutaloxycarbonyl) dehydroalanine benzyl ester (5) (960.0 mg, $2.54 \mathrm{mmol})$, Potassium phosphate dibasic (1107.2 mg, $6.35 \mathrm{mmol})$, tetraMeO-Acri-NdiMeOPh (163.2 mg, $0.256 \mathrm{mmol})$ and anhydrous DMF (24 mL).

The above stock solution was then stirred for 20 minutes under an atmosphere of nitrogen, then uncapped \& pipetted $1.5 \mathrm{~mL}$ of the solution containing bis-(tertbutaloxycarbonyl) dehydroalanine benzyl ester (5) (60.0 mg, 0.159 $\mathrm{mmol})$, Potassium phosphate dibasic $(69.2 \mathrm{mg}, 0.398 \mathrm{mmol})$, and tetraMeO-Acri- $\mathrm{N}$-diMeOPh (10.2 mg, $0.016 \mathrm{mmol})$ into $8 \mathrm{~mL}$ vials containing the acid monomers $(0.238 \mathrm{mmol})$. The reaction mixtures were capped and degassed by bubbling nitrogen through each solution (with vent needle) for 15 seconds before removing the needles. The sealed reaction mixtures were then irradiated with Blue LED light in the Aldrich's SynLED parallel photoreactor ${ }^{\circledR}$ (wavelength: 465-470 nm) at room temperature for $20 \mathrm{hrs}$ with stirring (sealed under an atmosphere of nitrogen). Followed by LC/MS. After $20 \mathrm{hrs}$ the reaction mixtures were diluted with $2 \mathrm{~mL}$ dioxane and stirred for 20 minutes. Reaction solutions were then filtered (syringe filter) and the filter was subsequently washed with $2 \times 2 \mathrm{~mL}$ dioxane. The combined filtrates were then further diluted with $2 \mathrm{~mL}$ dioxane, then concentrated in vacuo and used crude as is for the next step.

STEP 2: To each of the crude products $(0.159 \mathrm{mmol}$ theoretical yield) from the first step of the library was added 2 $\mathrm{mL} \mathrm{CH} \mathrm{Cl}_{2}$, then TFA ( $1.5 \mathrm{~mL}, 19.5 \mathrm{mmol}$ ). The reaction mixtures were then capped \& stirred at room temperature for 1.5 hours. Reaction mixtures were diluted with $5 \mathrm{~mL}$ of dioxane followed by $5 \mathrm{~mL}$ of toluene. The reaction mixtures were then concentrated. The resulting residue was then diluted to a total volume of $1.75 \mathrm{~mL}$ with DMSO, filtered (syringe filter) \& the filtrate was then purified by mass guided reverse phase purification (TFA modifier). The desired fractions were concentrated in vacuo to yield products.

\section{Monomers employed: total 15}<smiles>O=C(O)C12CCC(c3ccccc3)(C1)C2</smiles><smiles>O=C(O)C1CCC(F)(F)CO1</smiles><smiles>O=C(O)Cc1cccnc1</smiles><smiles>O=C(O)C1(F)CCCCC1</smiles><smiles>O=C(O)C1(c2ccc(Cl)cc2)CCC1</smiles><smiles>CN1CC(C(=O)O)CC1=O</smiles><smiles>N#CC1CCC(C(=O)O)CC1</smiles><smiles>O=C(O)C1CCC(F)(F)CC1</smiles><smiles>O=C(O)CC1CCC(F)(F)CC1</smiles><smiles>O=C(O)Cc1cn2ccccc2n1</smiles><smiles>O=C(O)C(F)(F)c1ccccc1</smiles><smiles>O=C(O)C1CCOCC1</smiles><smiles>CC1(C(=O)O)CCC(F)(F)CC1</smiles><smiles>O=C(O)Cc1ccc(Cl)cc1</smiles> 
13 monomers yielded intended products with $>85 \%$ HPLC purity. The isolated yields and characterization data are described below:

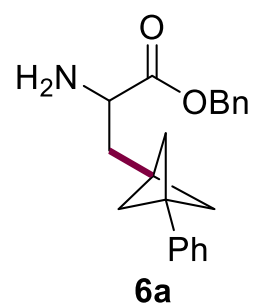

Following method A, 6 a was obtained as white solid ( $34.8 \mathrm{mg}, 0.080 \mathrm{mmol}, 50 \%$ yield, mono-TFA salt)

${ }^{1} \mathrm{H}$ NMR $\left(500 \mathrm{MHz}\right.$, Methanol- $\left.d_{4}\right) \delta 7.46(\mathrm{~d}, J=6.5 \mathrm{~Hz}, 2 \mathrm{H}), 7.43-7.35(\mathrm{~m}, 3 \mathrm{H}), 7.25(\mathrm{t}, J=7.4 \mathrm{~Hz}, 2 \mathrm{H}), 7.17(\mathrm{t}, J=$ $7.4 \mathrm{~Hz}, 1 \mathrm{H}), 7.14-7.10(\mathrm{~m}, 2 \mathrm{H}), 5.31(\mathrm{~d}, J=3.1 \mathrm{~Hz}, 2 \mathrm{H}), 4.11(\mathrm{dd}, J=6.8,5.2 \mathrm{~Hz}, 1 \mathrm{H}), 2.30-2.08(\mathrm{~m}, 2 \mathrm{H}), 1.94(\mathrm{~s}$, $6 \mathrm{H})$.

${ }^{13} \mathrm{C}$ NMR (126 MHz, MeOD) $\delta$ 170.5, 141.5, 136.2, 130.2, 129.9, 129.8, 129.2, 127.6, 126.8, 69.4, 53.6, 52.7, 43.3, $36.3,33.7$.

HRMS calcd for $\mathrm{C}_{21} \mathrm{H}_{23} \mathrm{NO}_{2}[\mathrm{M}+\mathrm{H}]^{+} 322.1802$ found 322.1790

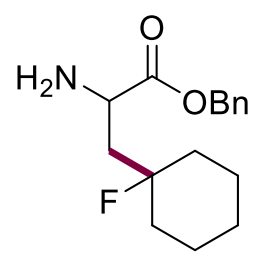

6b

Following method A, 6 b was obtained as white solid $(51.5 \mathrm{mg}, 0.131 \mathrm{mmol}, 82 \%$ yield, mono-TFA salt)

${ }^{1} \mathrm{H}$ NMR (600 MHz, MeOD) $\delta 7.43-7.32(\mathrm{~m}, 5 \mathrm{H}), 5.27(\mathrm{~s}, 2 \mathrm{H}), 4.32(\mathrm{dd}, \mathrm{J}=8.8,4.1 \mathrm{~Hz}, 1 \mathrm{H}), 2.29$ (ddd, J = 30.0, 15.5, $4.1 \mathrm{~Hz}, 1 \mathrm{H}), 2.12(\mathrm{ddd}, \mathrm{J}=15.5,14.0,8.8 \mathrm{~Hz}, 1 \mathrm{H}), 2.01-1.92(\mathrm{~m}, 1 \mathrm{H}), 1.85-1.78(\mathrm{~m}, 1 \mathrm{H}), 1.66-1.41(\mathrm{~m}, 7 \mathrm{H}), 1.34-$ $1.25(\mathrm{~m}, 1 \mathrm{H})$.

${ }^{13} \mathrm{C}$ NMR (126 MHz, MeOD) $\delta$ 169.2, 134.9, 128.5, 128.5, 128.3, 95.4 (d, J = 169.5 Hz), 68.1, 49.0, 39.7 (d, J = 21.3 $\mathrm{Hz}), 35.5(\mathrm{~d}, \mathrm{~J}=21.7 \mathrm{~Hz}), 33.3(\mathrm{~d}, \mathrm{~J}=22.2 \mathrm{~Hz}), 24.5,21.4(\mathrm{t}, \mathrm{J}=2.8 \mathrm{~Hz})$.

HRMS calcd for $\mathrm{C}_{16} \mathrm{H}_{23} \mathrm{FNO}_{2}[\mathrm{M}+\mathrm{H}]^{+} 280.1713$ found 280.1715 


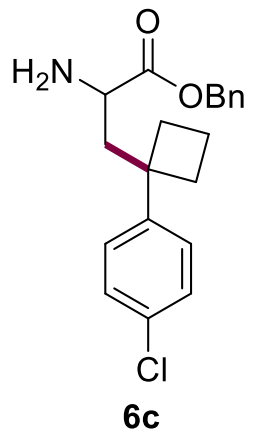

Following method A, $6 \mathrm{c}$ was obtained as white solid $(8.7 \mathrm{mg}, 0.019 \mathrm{mmol}, 12 \%$ yield, mono-TFA salt)

${ }^{1} \mathrm{H}$ NMR $(500 \mathrm{MHz}, \mathrm{MeOD})$ 8 7.44-7.34 (m, 5H), $7.26(\mathrm{~d}, \mathrm{~J}=8.2 \mathrm{~Hz}, 2 \mathrm{H}), 7.18(\mathrm{~d}, \mathrm{~J}=8.2 \mathrm{~Hz}, 2 \mathrm{H}), 5.13(\mathrm{~d}, \mathrm{~J}=12.0 \mathrm{~Hz}$, $1 \mathrm{H}), 5.00(\mathrm{~d}, \mathrm{~J}=12.0 \mathrm{~Hz}, 1 \mathrm{H}), 3.44(\mathrm{dd}, \mathrm{J}=9.1,4.3 \mathrm{~Hz}, 1 \mathrm{H}), 2.55(\mathrm{dd}, \mathrm{J}=14.1,9.3 \mathrm{~Hz}, 1 \mathrm{H}), 2.41-2.26(\mathrm{~m}, 3 \mathrm{H}), 2.25-$ $2.18(\mathrm{~m}, 1 \mathrm{H}), 2.08-1.94(\mathrm{~m}, 2 \mathrm{H}), 1.86-1.75(\mathrm{~m}, 1 \mathrm{H})$.

${ }^{13} \mathrm{C}$ NMR $(126 \mathrm{MHz}$, MeOD) $\delta$ 170.5, 146.4, 135.9, 133.1, 130.1, 129.9, 129.7, 129.6, 129.1, 69.2, 51.8, 45.8, 44.3, 35.17, 33.8, 16.6.

HRMS calcd for $\mathrm{C}_{20} \mathrm{H}_{23} \mathrm{CINO}_{2}[\mathrm{M}+\mathrm{H}]^{+} 344.1417$ found 344.1418

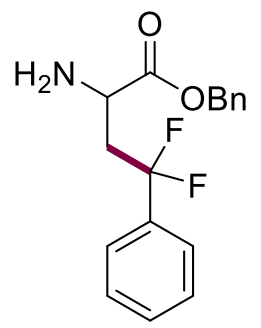

$6 d$

Following method A, 6d was obtained as white solid ( $2.6 \mathrm{mg}, 0.006 \mathrm{mmol}, 4 \%$ yield, mono-TFA salt)

${ }^{1} \mathrm{H}$ NMR $\left(500 \mathrm{MHz}\right.$, DMSO- $\left.d_{6}\right) \delta 8.76(\mathrm{~s}, 3 \mathrm{H}), 7.59-7.48(\mathrm{~m}, 5 \mathrm{H}), 7.46-7.35(\mathrm{~m}, 5 \mathrm{H}), 5.22(\mathrm{~d}, J=12.4 \mathrm{~Hz}, 1 \mathrm{H}), 5.11$ $(\mathrm{d}, J=12.4 \mathrm{~Hz}, 1 \mathrm{H}), 4.28(\mathrm{t}, J=5.9 \mathrm{~Hz}, 1 \mathrm{H}), 2.90$ (ddd, $J=20.6,14.2,5.8 \mathrm{~Hz}, 2 \mathrm{H})$.

${ }^{13} \mathrm{C}$ NMR (126 MHz, DMSO) $\delta$ 168.7, 135.3, 131.2, 129.4, 128.9, 128.9, 128.8, 125.3, 125.2, $125.2,121.9$ (t, J = 242.6 $\mathrm{Hz}), 67.9,48.2,38.9(\mathrm{t}, J=27.3 \mathrm{~Hz})$.

HRMS calcd for $\mathrm{C}_{17} \mathrm{H}_{17} \mathrm{~F}_{2} \mathrm{NO}_{2} \mathrm{Na}[\mathrm{M}+\mathrm{Na}]^{+} 328.1120$ found 328.1122 


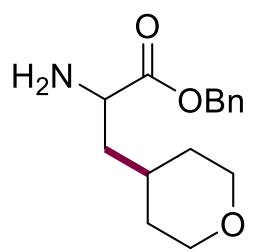

$6 e$

Following method A, 6e was obtained as white solid $(51.7 \mathrm{mg}, 0.137 \mathrm{mmol}, 86 \%$ yield, mono-TFA salt)

${ }^{1} \mathrm{H}$ NMR $\left(500 \mathrm{MHz}, \mathrm{CDCl}_{3}\right) \delta 7.39-7.34(\mathrm{~m}, 3 \mathrm{H}), 7.33-7.29(\mathrm{~m}, 2 \mathrm{H}), 5.24(\mathrm{~d}, \mathrm{~J}=12.0 \mathrm{~Hz}, 1 \mathrm{H}), 5.11(\mathrm{~d}, \mathrm{~J}=12.0 \mathrm{~Hz}$, $1 \mathrm{H}), 4.03(\mathrm{t}, \mathrm{J}=7.0 \mathrm{~Hz}, 1 \mathrm{H}), 3.91-3.80(\mathrm{~m}, 2 \mathrm{H}), 3.30-3.18(\mathrm{~m}, 2 \mathrm{H}), 1.82(\mathrm{t}, \mathrm{J}=7.0 \mathrm{~Hz}, 2 \mathrm{H}), 1.73-1.62(\mathrm{~m}, 1 \mathrm{H}), 1.56(\mathrm{~s}$, $1 \mathrm{H}), 1.53(\mathrm{~s}, 1 \mathrm{H}), 1.25-1.17(\mathrm{~m}, 2 \mathrm{H})$.

${ }^{13} \mathrm{C}$ NMR $(126 \mathrm{MHz}, \mathrm{MeOD}) \delta 170.8,136.4,130.1,129.9,129.8,69.2,68.5,68.5,51.4,38.7,33.6,33.5,32.1$.

HRMS calcd for $\mathrm{C}_{15} \mathrm{H}_{22} \mathrm{NO}_{3}[\mathrm{M}+\mathrm{H}]^{+} 264.1599$ found 264.1608

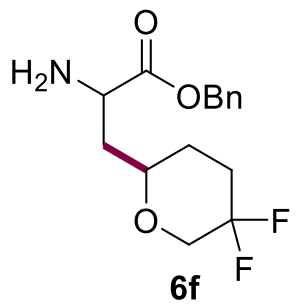

Following method A, $6 \mathrm{f}$ was obtained as 2:1 mixture of diastereomers $(55.5 \mathrm{mg}, 0.134 \mathrm{mmol}$, $84 \%$ yield, white solid, mono-TFA salt).

${ }^{1} \mathrm{H}$ NMR (600 MHz, MeOD, minor isomer is denoted by $\left.*\right) \delta 7.49-7.31(\mathrm{~m}, 5 \mathrm{H}$, overlapping peaks from both diastereomers), $5.37(\mathrm{~d}, J=11.9 \mathrm{~Hz}, 0.66 \mathrm{H}), 5.29 *(\mathrm{~d}, J=12.0 \mathrm{~Hz}, 0.33 \mathrm{H}), 5.27-5.22(\mathrm{~m}, 1 \mathrm{H}$, overlapping peaks from both diastereomers), $4.26(\mathrm{dd}, J=6.9,3.8 \mathrm{~Hz}, 0.66 \mathrm{H}), 4.21^{*}(\mathrm{dd}, J=7.2,5.2 \mathrm{~Hz}, 0.33 \mathrm{H}), 3.86-3.76(\mathrm{~m}, 1 \mathrm{H}$, overlapping peaks from both diastereomers), 3.65* $(\mathrm{t}, J=10.8 \mathrm{~Hz}, 0.33 \mathrm{H}), 3.48^{*}(\mathrm{ddd}, J=29.2,12.2,1.8 \mathrm{~Hz}, 0.33 \mathrm{H}$ ), $3.35-3.23(\mathrm{~m}, 1.33 \mathrm{H}), 2.22-1.51(\mathrm{~m}, 6 \mathrm{H}$, overlapping peaks from both diastereomers).

${ }^{13} \mathrm{C}$ NMR (126 MHz, MeOD, minor isomer is denoted by *) $\delta 170.1^{*}, 169.9,136.5,136.4^{*}, 130.2,129.98^{*}, 129.88$, 129.79*, 129.78, 129.70*, 119.2* (t, J = 244.1 Hz), $119.1(\mathrm{t}, \mathrm{J}=244.1 \mathrm{~Hz}), 75.4^{*}, 74.5,70.31^{*}(\mathrm{dd}, \mathrm{J}=35.9,27.8 \mathrm{~Hz})$, $70.26(\mathrm{dd}, J=35.9,27.9 \mathrm{~Hz}), 69.25,69.22 *, 52.5^{*}, 51.9,36.2^{*}, 35.7,31.94 *(\mathrm{dd}, J=25.0,22.1 \mathrm{~Hz}), 31.83$ (dd, $J=24.9$, $22.2 \mathrm{~Hz}$ ), 29.80* (d, J=8.5 Hz), 29.56 (d, $J=8.5 \mathrm{~Hz}$ ).

HRMS calcd for $\mathrm{C}_{15} \mathrm{H}_{20} \mathrm{~F}_{2} \mathrm{NO}_{3}[\mathrm{M}+\mathrm{H}]^{+} 300.1411$ found 300.1413 
<smiles>CN1CC(CC(N)C(=O)OCc2ccccc2)CC1=O</smiles>

Following method A, $6 \mathrm{~g}$ was obtained as $1: 1$ diastereomeric mixture $(11 \mathrm{mg}, 0.028 \mathrm{mmol}, 18 \%$ yield, colorless oil, mono-TFA salt)

${ }^{1} \mathrm{H}$ NMR $(500 \mathrm{MHz}, \mathrm{MeOD}$, *denotes other isomer) $\delta$ 7.46-7.34 (m, 5H, overlapping signals from both isomers), 5.36$5.26(\mathrm{~m}, 2 \mathrm{H}$, overlapping signals from both isomers $), 4.13-4.05(\mathrm{~m}, 1 \mathrm{H}$, overlapping signals from both isomers $), 3.50$ ( $q, J=7.4 \mathrm{~Hz}, 1 \mathrm{H}$, overlapping signals from both isomers), 3.11-3.05 ( $\mathrm{m}, 1 \mathrm{H}$, overlapping signals from both isomers), $2.78(\mathrm{~s}, 1.5 \mathrm{H}), 2.77^{*}(\mathrm{~s}, 1.5 \mathrm{H}), 2.55-2.45(\mathrm{~m}, 2 \mathrm{H}$, overlapping signals from both isomers $), 2.14-2.03(\mathrm{~m}, 2 \mathrm{H}$, overlapping signals from both isomers), 2.01-1.89 ( $\mathrm{m}, 1 \mathrm{H}$, overlapping signals from both isomers).

${ }^{13} \mathrm{C}$ NMR $(126 \mathrm{MHz}, \mathrm{MeOD}, *$ denotes another isomer) $\delta 174.52,174.48 *, 168.83,134.86 *, 134.83,128.65 *, 128.61$, $128.58,128.4,68.02,54.4,54.1 *, 51.12,51.05^{*}, 36.4^{*}, 36.3,34.77,34.73^{*}, 28.36^{*}, 28.34,28.04,27.96^{*}$

HRMS calcd for $\mathrm{C}_{15} \mathrm{H}_{21} \mathrm{~N}_{2} \mathrm{O}_{3}[\mathrm{M}+\mathrm{H}]^{+} 277.1552$ found 277.1556

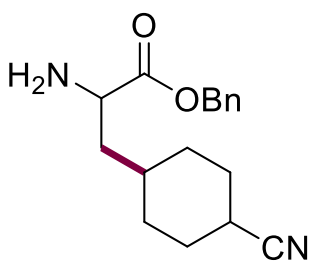

$6 \mathrm{~h}$

Following method $\mathrm{A}, 6 \mathrm{~h}$ was obtained as $\sim 1: 1$ diastereomeric mixture $(52 \mathrm{mg}, 0.130 \mathrm{mmol}, 82 \%$ yield, colorless oil, mono-TFA salt)

${ }^{1} \mathrm{H}$ NMR $\left(600 \mathrm{MHz}\right.$, Acetonitrile- $\left.d_{3}\right) \delta 8.08(\mathrm{~s}, 3 \mathrm{H}$, overlapping signals from both isomers), $7.43-7.33$ ( $\mathrm{m}, 5 \mathrm{H}$, overlapping signals from both isomers), 5.25 (d, $J=12.2 \mathrm{~Hz}, 1 \mathrm{H}$, overlapping signals from both isomers), 5.19-5.16 ( $\mathrm{m}, 1 \mathrm{H}$, overlapping signals from both isomers), 4.08 (dt, $J=19.0,7.2 \mathrm{~Hz}, 1 \mathrm{H}$, overlapping signals from both isomers), $2.95(\mathrm{t}, J=3.8 \mathrm{~Hz}, 0.5 \mathrm{H}), 2.43(\mathrm{tt}, J=12.2,3.7 \mathrm{~Hz}, 0.5 \mathrm{H}), 2.04-1.96(\mathrm{~m}, 1 \mathrm{H}$, overlapping signals from both isomers), 1.84 (ddd, $J=18.3,7.3,3.8 \mathrm{~Hz}, 2 \mathrm{H}$, overlapping signals from both isomers), 1.75 (q, $J=9.7,8.5 \mathrm{~Hz}, 2 \mathrm{H}$, overlapping signals from both isomers), 1.69 (ddd, $J=13.2,8.5,3.9 \mathrm{~Hz}, 1 \mathrm{H}$, overlapping signals from both isomers), $1.54-1.33$ (m, $3 \mathrm{H}$, overlapping signals from both isomers), 1.21 (dtd, $J=17.1,10.7,8.5,4.7 \mathrm{~Hz}, 1 \mathrm{H}$, overlapping signals from both isomers), 0.93 (dddd, $J=21.4,12.9,8.1,3.4 \mathrm{~Hz}, 1 \mathrm{H}$, overlapping signals from both isomers).

${ }^{13} \mathrm{C}$ NMR (151 MHz, Acetonitrile- $d_{3}$, *denotes another isomer) $\delta$ 170.7, 170.6*, 136.09, 136.09*, 129.6*, 129.5, 129.51*, 129.47, 129.42, 123.8*, 123.1, 68.7, 51.7, 37.98, 37.93*, 33.1*, 32.8, 31.5, 31.4, 29.97, 29.89, 29.14*, $29.11^{*}, 28.4^{*}, 28.32,28.27^{*}, 27.5^{*}$.

HRMS calcd for $\mathrm{C}_{17} \mathrm{H}_{23} \mathrm{~N}_{2} \mathrm{O}_{2}[\mathrm{M}+\mathrm{H}]^{+} 287.1759$ found 287.1767 


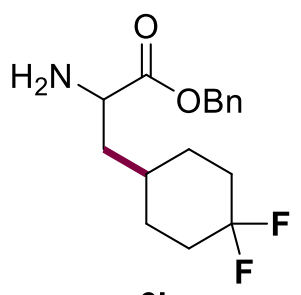

$6 i$

Following method A, 6i was obtained as colorless oil (54.2 mg, $0.132 \mathrm{mmol}, 83 \%$ yield, mono-TFA salt)

${ }^{1} \mathrm{H}$ NMR (500 MHz, MeOD) $\delta 7.46-7.30(\mathrm{~m}, 5 \mathrm{H}), 5.37(\mathrm{~d}, J=11.9 \mathrm{~Hz}, 1 \mathrm{H}), 5.23(\mathrm{~d}, J=11.9 \mathrm{~Hz}, 1 \mathrm{H}), 4.10(\mathrm{t}, J=7.2$ $\mathrm{Hz}, 1 \mathrm{H}), 2.02-1.91(\mathrm{~m}, 2 \mathrm{H}), 1.89-1.51(\mathrm{~m}, 6 \mathrm{H}), 1.50-1.42(\mathrm{~m}, 1 \mathrm{H}), 1.29-1.21(\mathrm{~m}, 2 \mathrm{H})$.

${ }^{13} \mathrm{C}$ NMR (126 MHz, MeOD) $\delta 170.7,136.4,130.1,129.9,129.8,124.2$ (dd, J = 241.5, 238.7 Hz), 69.2, 52.0, 37.8 (d, J $=2.6 \mathrm{~Hz}$ ), 34.1 (ddd, $J=26.1,23.3,3.3 \mathrm{~Hz}), 32.9,29.6$ (dd, J = 41.4, $9.8 \mathrm{~Hz}$ ).

HRMS calcd for $\mathrm{C}_{16} \mathrm{H}_{22} \mathrm{~F}_{2} \mathrm{NO}_{2}[\mathrm{M}+\mathrm{H}]^{+} 298.1618$ found 298.1623

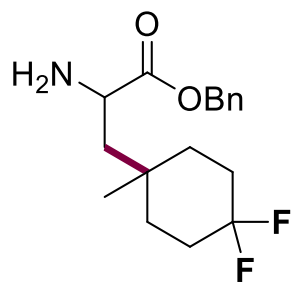

6j

Following method A, 6j was obtained as colorless oil (45.8 mg, $0.108 \mathrm{mmol}, 68 \%$ yield, mono-TFA salt)

${ }^{1} \mathrm{H}$ NMR (500 MHz, MeOD) $\delta 7.48-7.32(\mathrm{~m}, 5 \mathrm{H}), 5.28(\mathrm{q}, \mathrm{J}=11.9 \mathrm{~Hz}, 2 \mathrm{H}), 4.10-4.04(\mathrm{~m}, 1 \mathrm{H}), 2.10-2.02(\mathrm{~m}, 1 \mathrm{H}), 1.94-$ $1.71(\mathrm{~m}, 4 \mathrm{H}), 1.67-1.59(\mathrm{~m}, 1 \mathrm{H}), 1.55-1.48(\mathrm{~m}, 2 \mathrm{H}), 1.47-1.40(\mathrm{~m}, 2 \mathrm{H}), 1.01(\mathrm{~s}, 3 \mathrm{H})$.

${ }^{13} \mathrm{C}$ NMR (126 MHz, MeOD) $\delta 171.2,136.0,130.2,129.9,129.8,124.2(\mathrm{t}, \mathrm{J}=240.2 \mathrm{~Hz}), 69.5,50.9,43.3,35.2(\mathrm{t}, \mathrm{J}=$ $4.8 \mathrm{~Hz}$ ), $34.3(\mathrm{t}, \mathrm{J}=4.8 \mathrm{~Hz}), 32.9,30.7(\mathrm{td}, J=24.4,2.4 \mathrm{~Hz}), 23.0$.

HRMS calcd for $\mathrm{C}_{17} \mathrm{H}_{24} \mathrm{~F}_{2} \mathrm{NO}_{2}[\mathrm{M}+\mathrm{H}]^{+} 312.1775$ found 312.1777 


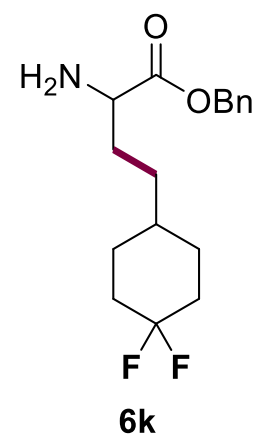

Following method A, 6k was obtained as oil (45.1 mg, $0.106 \mathrm{mmol}, 67 \%$ yield, mono-TFA salt)

${ }^{1} \mathrm{H}$ NMR $(500 \mathrm{MHz}, \mathrm{MeOD})$ 8 7.44-7.34 (m, 5H), $5.38(\mathrm{~d}, \mathrm{~J}=11.9 \mathrm{~Hz}, 1 \mathrm{H}), 5.21(\mathrm{~d}, \mathrm{~J}=11.9 \mathrm{~Hz}, 1 \mathrm{H}), 4.08(\mathrm{t}, \mathrm{J}=6.1 \mathrm{~Hz}$, $1 \mathrm{H}), 2.02-1.85(\mathrm{~m}, 4 \mathrm{H}), 1.77-1.61(\mathrm{~m}, 4 \mathrm{H}), 1.37-1.26(\mathrm{~m}, 2 \mathrm{H}), 1.20-1.05(\mathrm{~m}, 3 \mathrm{H})$.

${ }^{13} \mathrm{C}$ NMR (126 MHz, MeOD) $\delta$ 170.43, 136.48, 130.05, 129.93, 129.75, 124.57 (dd, J = 241.3, 238.7 Hz), 69.08, 53.97, 36.33, 34.22 (dd, J = 25.1, 23.1 Hz), 31.42 (d, J = 2.4 Hz), 29.71 (dd, J = 9.5, 6.8 Hz), 29.19.

HRMS calcd for $\mathrm{C}_{17} \mathrm{H}_{24} \mathrm{~F}_{2} \mathrm{NO}_{2}[\mathrm{M}+\mathrm{H}]^{+} 312.1775$ found 312.1769

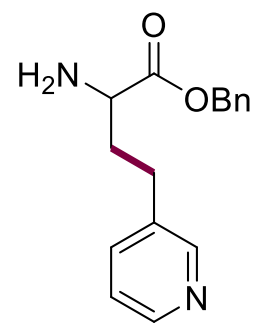

6I

Following method A, 6I was obtained as white solid ( $9.5 \mathrm{mg}, 0.019 \mathrm{mmol}, 12 \%$ yield, bis-TFA salt)

${ }^{1} \mathrm{H}$ NMR (500 MHz, MeOD) $\delta 8.56(\mathrm{~d}, \mathrm{~J}=4.7 \mathrm{~Hz}, 1 \mathrm{H}), 8.49(\mathrm{~s}, 1 \mathrm{H}), 8.01(\mathrm{~d}, \mathrm{~J}=8.0 \mathrm{~Hz}, 1 \mathrm{H}), 7.67(\mathrm{dd}, \mathrm{J}=7.8,5.4 \mathrm{~Hz}$, $1 \mathrm{H}), 7.52-7.43(\mathrm{~m}, 2 \mathrm{H}), 7.43-7.34(\mathrm{~m}, 3 \mathrm{H}), 5.35(\mathrm{~d}, \mathrm{~J}=11.9 \mathrm{~Hz}, 1 \mathrm{H}), 5.27(\mathrm{~d}, \mathrm{~J}=11.9 \mathrm{~Hz}, 1 \mathrm{H}), 4.15(\mathrm{t}, \mathrm{J}=6.3 \mathrm{~Hz}$, $1 \mathrm{H}), 2.95-2.86(\mathrm{~m}, 1 \mathrm{H}), 2.81-2.72(\mathrm{~m}, 1 \mathrm{H}), 2.33-2.15(\mathrm{~m}, 2 \mathrm{H})$.

${ }^{13} \mathrm{C}$ NMR (126 MHz, MeOD) $\delta 170.0,146.5,145.1,142.7,139.6,136.4,130.1,130.0,129.8,126.8,69.4,53.3,32.5$, 28.9 .

HRMS calcd for $\mathrm{C}_{16} \mathrm{H}_{19} \mathrm{~N}_{2} \mathrm{O}_{2}[\mathrm{M}+\mathrm{H}]^{+} 271.1446$ found 271.1441 


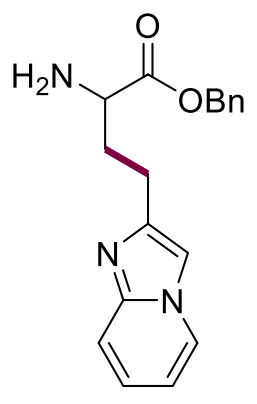

$6 \mathrm{~m}$

Following method A, $6 \mathrm{~m}$ was obtained as semi-solid $(50.3 \mathrm{mg}, 0.093 \mathrm{mmol}, 59 \%$ yield, bis-TFA salt)

${ }^{1} \mathrm{H}$ NMR (500 MHz, MeOD) $\delta 8.67(\mathrm{~d}, J=6.8 \mathrm{~Hz}, 1 \mathrm{H}), 7.96(\mathrm{~s}, 1 \mathrm{H}), 7.91-7.86(\mathrm{~m}, 1 \mathrm{H}), 7.85-7.80(\mathrm{~m}, 1 \mathrm{H}), 7.46-7.31$ $(\mathrm{m}, 6 \mathrm{H}), 5.31(\mathrm{~s}, 2 \mathrm{H}), 4.23(\mathrm{t}, J=6.5 \mathrm{~Hz}, 1 \mathrm{H}), 3.11(\mathrm{ddd}, J=15.6,9.6,6.1 \mathrm{~Hz}, 1 \mathrm{H}), 3.03(\mathrm{ddd}, J=15.7,9.4,6.6 \mathrm{~Hz}, 1 \mathrm{H})$, $2.51-2.29(\mathrm{~m}, 2 \mathrm{H})$.

${ }^{13} \mathrm{C}$ NMR (126 MHz, MeOD) $\delta$ 169.9, 141.9, 137.6, 136.3, 134.2, 129.9, 129.9, 129.8, 129.8, 118.1, 113.6, 113.2, $69.4,53.1,30.0,22.3$.

HRMS calcd for $\mathrm{C}_{18} \mathrm{H}_{20} \mathrm{~N}_{3} \mathrm{O}_{2}[\mathrm{M}+\mathrm{H}]^{+} 310.1555$ found 310.1555 
C. Batch synthesis of $\alpha$-amino benzyl esters $6 \mathrm{~d}, 6 \mathrm{n}-6 \mathrm{t}$ (Table 3 )

Method B: General procedure for batch conditions

\section{$\mathbf{R}^{1} \mathrm{COOH}(7)$}

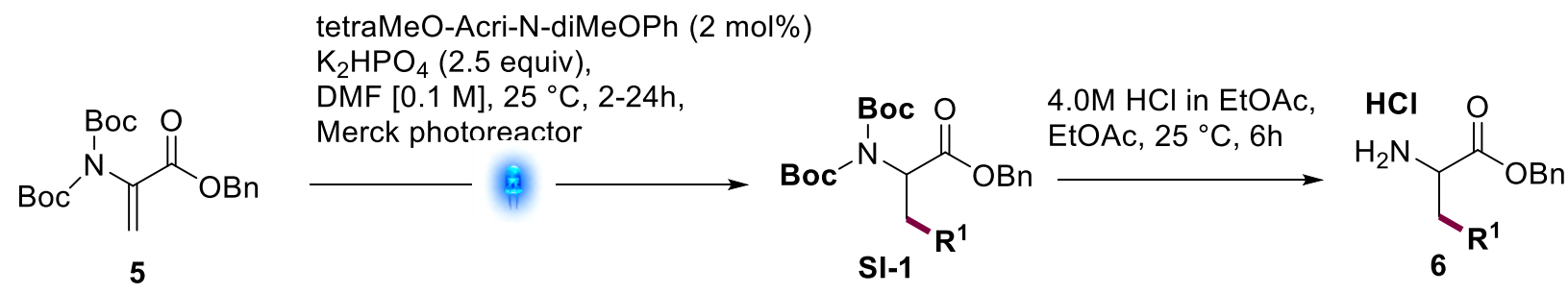

\section{Synthesis of bisBoc adduct SI-1}

In a 2-dram vial were added benzyl 2-(bis(tert-butaloxycarbonyl)amino)acrylate (5) (100-150 mg, 1.0 equiv), carboxylic acid 7 ( 1.5 equiv), and tetraMeO-Acri-N-diMeoPh (0.02 equiv). DMF ( $0.13 \mathrm{M}$ ) was added to the vials followed by potassium phosphate dibasic (3.0 equiv). Vial was sealed and then set on the PennOC Photoreactor ${ }^{\circledR}$ (wavelength: $450 \mathrm{~nm}$; LED intensity: 100\%; fan speed: $5000 \mathrm{rpm}$; stir: 1000 rpm) (2-24 h). After the reaction was complete, DMSO $(1 \mathrm{~mL})$ was added to the mixture, which was filtered and the filtrate purified by reverse phase (C18) HPLC ( $30 \times 150 \mathrm{~mm}$, Waters SunFire ${ }^{\circledR} \mathrm{OBD}^{\mathrm{TM}} 10 \mathrm{mM}$ ) eluting with a gradient of 5-95\% Acetonitrile/Water $+0.1 \%$ TFA over 15 minutes at $43 \mathrm{ml} / \mathrm{min}$. The fractions that contained product were combined, neutralized with $1 \mathrm{ml}$ $\mathrm{NH} 4 \mathrm{OH}$, and concentrated. The residue was diluted with sat $\mathrm{NaHCO}_{3}$ and than extracted with $\mathrm{CH}_{2} \mathrm{Cl} 2$. The organic portion was separated, washed with brine, dried over anhydrous $\mathrm{MgSO}$, filtered, and the volatiles were removed under reduced pressure to provide bisBoc adduct SI-1.

\section{Synthesis of $\alpha$-amino benzyl ester hydrochloride (6):}

SI-1 (1.0 equiv) was treated with $2.0 \mathrm{ml}$ of saturated $\mathrm{HCl}$ in EtOAc. The mixture was stirred for 6.0 hours. After the reaction was completed, volatiles were removed under reduced pressure. Added EtOAc $(5 \mathrm{ml})$, stirred 5 minutes, and then the volatiles were removed under reduce pressure to provide $\alpha$-amino benzyl ester hydrochloride 6 as a white solid or colorless oil.

Yields are provided for $1^{\text {st }}$ step, $2^{\text {nd }}$ step and over two steps. 

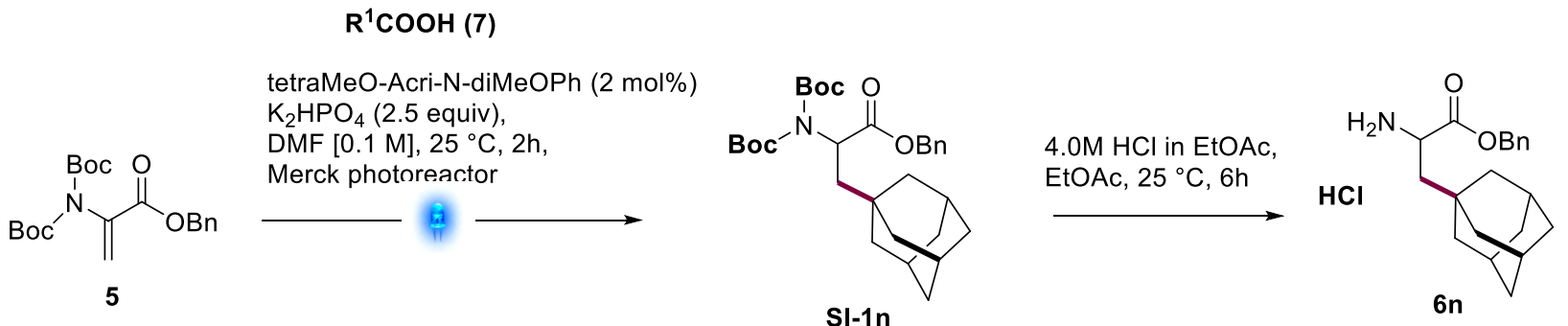

Prepared by Method B using 1-adamantanecarboxylic acid $(72 \mathrm{mg}, 0.397 \mathrm{mmol}, 1.5 \mathrm{eq}$ ) for 2 hours on PennOC Photoreactor ${ }^{\circledR}$.

Obtained SI-1n as yellow oil (115 mg, $0.224 \mathrm{mmol}, 85 \%$ yield).

${ }^{1} \mathrm{H}$ NMR (500 MHz, MeOD) $\delta 7.37-7.27(\mathrm{~m}, 5 \mathrm{H}), 5.12(\mathrm{q}, J=12.4 \mathrm{~Hz}, 2 \mathrm{H}), 5.01$ (dd, $\left.J=8.3,3.1 \mathrm{~Hz}, 1 \mathrm{H}\right), 2.05(\mathrm{dd}, J=$ 15.2, 3.1 Hz, 1H), $1.94(\mathrm{~s}, 3 \mathrm{H}), 1.75(\mathrm{~d}, J=12.1 \mathrm{~Hz}, 3 \mathrm{H}), 1.66(\mathrm{~d}, J=11.7 \mathrm{~Hz}, 3 \mathrm{H}), 1.61-1.49(\mathrm{~m}, 7 \mathrm{H}), 1.43(\mathrm{~s}, 18 \mathrm{H})$.

${ }^{13} \mathrm{C}$ NMR (126 MHz, MeOD) $\delta$ 172.8, 153.6, 137.3, 129.5, 129.3, 129.2, 84.5, 68.1, 55.6, 45.1, 43.4, 38.0, 33.2, 30.1, 28.3.

HRMS calcd for $\mathrm{C}_{30} \mathrm{H}_{43} \mathrm{NO}_{6}[\mathrm{M}+\mathrm{Na}]^{+} 536.2983$ found 536.2980

SI-1n (98 mg, $0.191 \mathrm{mmol}$ ) was subjected to bisBoc deprotection affording $6 \mathrm{n}$ as white solid $(63 \mathrm{mg}, 0.180 \mathrm{mmol}$, $93 \%$ yield)

${ }^{1} \mathrm{H}$ NMR $(500 \mathrm{MHz}, \mathrm{MeOD}) \delta 7.60-7.17(\mathrm{~m}, 5 \mathrm{H}), 5.42-5.12(\mathrm{~m}, 2 \mathrm{H}), 4.06$ (dd, J = 6.9, $\left.4.9 \mathrm{~Hz}, 1 \mathrm{H}\right), 1.92(\mathrm{~s}, 3 \mathrm{H}), 1.79$ (dd, $J=14.7,6.9 \mathrm{~Hz}, 1 \mathrm{H}), 1.72(\mathrm{~d}, J=12.2 \mathrm{~Hz}, 3 \mathrm{H}), 1.62(\mathrm{~d}, J=11.8 \mathrm{~Hz}, 3 \mathrm{H}), 1.57-1.45(\mathrm{~m}, 7 \mathrm{H})$.

${ }^{13} \mathrm{C}$ NMR (126 MHz, MeOD) $\delta$ 171.5, 136.2, 130.1, 129.9, 129.7, 69.3, 50.1, 46.4, 42.9, 37.6, 33.2, 29.8.

HRMS calcd for $\mathrm{C}_{20} \mathrm{H}_{27} \mathrm{NO}_{2}[\mathrm{M}+\mathrm{H}]^{+} 314.2115$ found 314.2107

\section{Hence, $6 \mathrm{n}$ was obtained in $79 \%$ yield over two steps.}




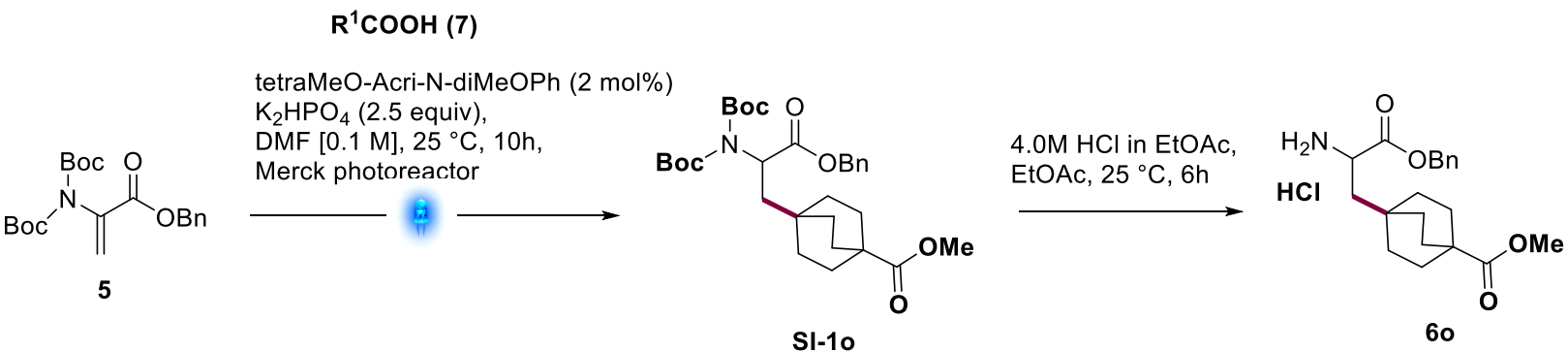

Prepared by Method B using benzyl 2-(bis(tert-butaloxycarbonyl)amino)acrylate (5) (150 mg, 0.397 mmol, 1 equiv) and 4-(methoxycarbonyl)bicyclo[2.2.2]octane-1-carboxylic acid (127 mg, $0.596 \mathrm{mmol}, 1.5 \mathrm{eq}$ ) for 10 hours on PennOC Photoreactor ${ }^{\circledR}$.

Obtained SI-10 as colorless oil (172 mg, $0.315 \mathrm{mmol}, 79 \%$ yield).

${ }^{1} \mathrm{H}$ NMR $(500 \mathrm{MHz}, \mathrm{MeOD}) \delta 7.60-7.14(\mathrm{~m}, 5 \mathrm{H}), 5.22-5.03(\mathrm{~m}, 2 \mathrm{H}), 4.96(\mathrm{dd}, J=8.5,3.1 \mathrm{~Hz}, 1 \mathrm{H}), 2.10$ (dd, $J=15.3$, $3.1 \mathrm{~Hz}, 1 \mathrm{H}), 1.77(\mathrm{t}, J=7.9 \mathrm{~Hz}, 6 \mathrm{H}), 1.64(\mathrm{dd}, J=15.3,8.5 \mathrm{~Hz}, 1 \mathrm{H}), 1.43(\mathrm{~s}, 23 \mathrm{H})$;

${ }^{13} \mathrm{C}$ NMR (126 MHz, MeOD) $\delta$ 179.9, 172.6, 153.6, 137.2, 129.5, 129.3, 129.2, 84.6, 68.1, 56.4, 52.2, 41.7, 40.1, 31.4, $31.3,29.5,28.2$

HRMS calcd for $\mathrm{C}_{30} \mathrm{H}_{43} \mathrm{NO}_{8} \mathrm{Na}[\mathrm{M}+\mathrm{Na}]^{+} 568.2881$ found 568.2889

SI-10 (172 mg, $0.315 \mathrm{mmol}$ ) was subjected to bisBoc deprotection affording 60 as white solid $(120 \mathrm{mg}, 0.314 \mathrm{mmol}$, $100 \%$ yield).

${ }^{1} \mathrm{H}$ NMR $(500 \mathrm{MHz}, \mathrm{MeOD}) \delta 7.57-7.29(\mathrm{~m}, 5 \mathrm{H}), 5.50-5.09(\mathrm{~m}, 2 \mathrm{H}), 4.03(\mathrm{dd}, J=7.0,5.0 \mathrm{~Hz}, 1 \mathrm{H}), 3.61(\mathrm{~s}, 3 \mathrm{H}), 1.85$ (dd, $J=14.8,7.1 \mathrm{~Hz}, 1 \mathrm{H}), 1.81-1.67(\mathrm{~m}, 6 \mathrm{H}), 1.53(\mathrm{dd}, J=14.8,4.8 \mathrm{~Hz}, 1 \mathrm{H}), 1.49-1.35(\mathrm{~m}, 6 \mathrm{H})$;

${ }^{13} \mathrm{C}$ NMR (126 MHz, MeOD) $\delta$ 179.6, 171.2, 136.1, 130.1, 129.9, 129.7, 69.4, 52.2, 50.8, 43.2, 39.9, 31.5, 31.1, 29.2

HRMS calcd for $\mathrm{C}_{20} \mathrm{H}_{28} \mathrm{NO}_{4}[\mathrm{M}+\mathrm{H}]^{+} 346.2013$ found 346.2004

Hence, 60 was obtained in $79 \%$ yield over two steps. 


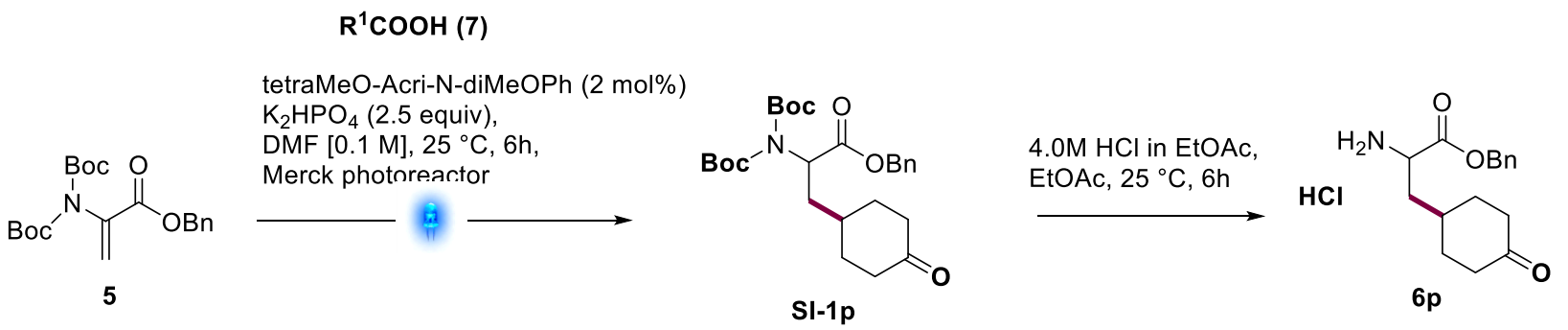

Prepared by Method B using 4-oxocyclohexanecarboxylic acid (57 mg, 0.397mmol, 1.5 eq) for 6 hours on PennOC Photoreactor ${ }^{\circledR}$.

Obtained SI-1p as yellow oil (110 mg, $0.232 \mathrm{mmol}, 87 \%$ yield). SI-1p was found to be a mixture of ketone (majority) and hydrate (very minor) forms in methanol-d4.

${ }^{1} \mathrm{H}$ NMR $(500 \mathrm{MHz}, \mathrm{MeOD}) \delta 7.52-7.26(\mathrm{~m}, 5 \mathrm{H}), 5.15(\mathrm{q}, J=12.4 \mathrm{~Hz}, 2 \mathrm{H}), 5.04(\mathrm{dd}, J=9.7,5.0 \mathrm{~Hz}, 1 \mathrm{H}), 2.48-2.28$ (m, 3H), 2.18 (ddd, $J=12.8,5.9,3.0 \mathrm{~Hz}, 1 \mathrm{H}), 2.08(\mathrm{ddd}, J=14.2,8.9,5.0 \mathrm{~Hz}, 1 \mathrm{H}), 2.05-1.88(\mathrm{~m}, 2 \mathrm{H}), 1.85-1.76(\mathrm{~m}$, $1 \mathrm{H}), 1.54-1.35(\mathrm{~m}, 3 \mathrm{H}), 1.44(\mathrm{~s}, 18 \mathrm{H})$.

${ }^{13} \mathrm{C}$ NMR (126 MHz, MeOD) $\delta$ 214.3, 172.1, 153.8, 137.2, 129.6, 129.3, 129.3, 129.2, 84.6, 68.0, 57.7, 41.5, 41.2, $36.4,34.39,34.29,32.9,28.2$.

HRMS calcd for $\mathrm{C}_{26} \mathrm{H}_{37} \mathrm{NO}_{7} \mathrm{Na}[\mathrm{M}+\mathrm{Na}]^{+} 498.2462$ found 498.2470

SI-1p (90 mg, $0.189 \mathrm{mmol}$ ) was subjected to bisBoc deprotection affording $6 \mathrm{p}$ as white solid $(56 \mathrm{mg}, 0.180 \mathrm{mmol}$, $95 \%$ yield).

${ }^{1} \mathrm{H}$ NMR $\left(500 \mathrm{MHz}, \mathrm{DMSO}-d_{6}\right) \delta 8.66(\mathrm{~s}, 3 \mathrm{H}), 7.41$ (ddd $\left.J=21.3,14.5,7.0 \mathrm{~Hz}, 5 \mathrm{H}\right), 5.26(\mathrm{q}, J=12.3 \mathrm{~Hz}, 2 \mathrm{H}), 4.12(\mathrm{t}, J$ $=6.7 \mathrm{~Hz}, 1 \mathrm{H}), 2.28(\mathrm{dtd}, J=40.2,13.7,5.7 \mathrm{~Hz}, 2 \mathrm{H}), 2.15(\mathrm{~d}, J=14.3 \mathrm{~Hz}, 2 \mathrm{H}), 2.00-1.85(\mathrm{~m}, 3 \mathrm{H}), 1.78(\mathrm{t}, J=6.9 \mathrm{~Hz}$, $2 \mathrm{H}), 1.32(\mathrm{dt}, J=10.9,5.1 \mathrm{~Hz}, 2 \mathrm{H})$.

${ }^{13} \mathrm{C}$ NMR $\left(126 \mathrm{MHz}, \mathrm{DMSO}-d_{6}\right) \delta$ 210.82, 170.2, 135.6, 129.0, 128.9, 128.9, 67.7, 50.7, 36.3, 31.9, 31.9, 31.6.

HRMS calcd for $\mathrm{C}_{16} \mathrm{H}_{22} \mathrm{NO}_{3}[\mathrm{M}+\mathrm{H}]^{+} 276.1594$ found 276.1591

\section{Hence, $6 p$ was obtained in $83 \%$ yield over two steps.}




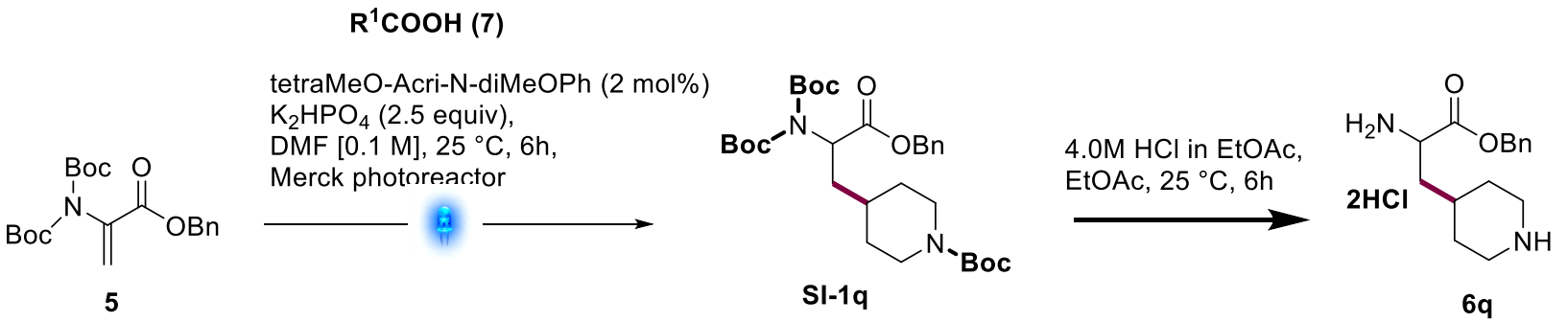

Prepared by Method B using 1-(tert-butaloxycarbonyl)piperdine-4-carboxylic acid ( $91 \mathrm{mg}, 0.397 \mathrm{mmol}, 1.5 \mathrm{eq}$ ) for 6 hours on PennOC Photoreactor ${ }^{\circledR}$.

Obtained SI-1q as yellow oil (140 mg, $0.249 \mathrm{mmol}, 94 \%$ yield).

${ }^{1} \mathrm{H}$ NMR $(500 \mathrm{MHz}, \mathrm{MeOD}) \delta 7.48-7.22(\mathrm{~m}, 5 \mathrm{H}), 5.26-5.07(\mathrm{~m}, 2 \mathrm{H}), 5.01$ (dd, $\left.J=9.8,4.9 \mathrm{~Hz}, 1 \mathrm{H}\right), 4.04(\mathrm{~d}, J=13.2$ $\mathrm{Hz}, 2 \mathrm{H}), 2.70(\mathrm{~s}, 2 \mathrm{H}), 2.00$ (ddd, $J=14.1,8.9,4.9 \mathrm{~Hz}, 1 \mathrm{H}), 1.88(\mathrm{td}, J=9.8,4.9 \mathrm{~Hz}, 1 \mathrm{H}), 1.81(\mathrm{~d}, J=12.7 \mathrm{~Hz}, 1 \mathrm{H}), 1.62$ $(\mathrm{d}, J=12.8 \mathrm{~Hz}, 1 \mathrm{H}), 1.50-1.47(\mathrm{~m}, 1 \mathrm{H}), 1.44(, 9 \mathrm{H}), 1.43(\mathrm{~s}, 18 \mathrm{H}), 1.15(\mathrm{qd}, J=12.7,4.4 \mathrm{~Hz}, 1 \mathrm{H}), 1.06$ (qd, $J=12.7,4.4$ $\mathrm{Hz}, 1 \mathrm{H})$.

${ }^{13} \mathrm{C}$ NMR $(126 \mathrm{MHz}, \mathrm{MeOD}) \delta$ 172.2, 156.5, 153.8, 137.2, 129.5, 129.3, 129.2, 84.6, 80.9, 68.0, 57.2, 37.3, 34.3, 28.7, 28.2.

HRMS calcd for $\mathrm{C}_{30} \mathrm{H}_{46} \mathrm{~N}_{2} \mathrm{O}_{8} \mathrm{Na}[\mathrm{M}+\mathrm{Na}]^{+} 585.3146$ found 585.3157

SI-1q (120 mg, $0.213 \mathrm{mmol}$ ) was subjected to bisBoc deprotection affording $\mathbf{6 q}$ as white solid $(74 \mathrm{mg}, 0.203 \mathrm{mmol}$, $95 \%$ yield).

${ }^{1} \mathrm{H}$ NMR $\left(500 \mathrm{MHz}, \mathrm{DMSO}-d_{6}\right) \delta 9.14(\mathrm{~s}, 1 \mathrm{H}), 9.01(\mathrm{~s}, 1 \mathrm{H}), 8.75(\mathrm{~s}, 3 \mathrm{H}), 7.41$ (ddd, $\left.J=20.8,13.9,7.0 \mathrm{~Hz}, 5 \mathrm{H}\right), 5.76(\mathrm{~s}$, $\mathrm{OH}), 5.24(\mathrm{~d}, J=2.4 \mathrm{~Hz}, 2 \mathrm{H}), 4.10(\mathrm{~s}, 1 \mathrm{H}), 3.20(\mathrm{~d}, J=12.3 \mathrm{~Hz}, 2 \mathrm{H}), 2.80-2.60(\mathrm{~m}, 2 \mathrm{H}), 1.89-1.65(\mathrm{~m}, 5 \mathrm{H}), 1.46-1.23$ $(\mathrm{m}, 2 \mathrm{H})$.

${ }^{13} \mathrm{C}$ NMR $(126 \mathrm{MHz}$, DMSO-d $)$ ) $\delta$ 169.99, 135.59, 128.99, 128.92, 128.84, 67.64, 55.41, 49.99, 43.25, 36.63, 29.75, $28.24,28.22$.

HRMS calcd for $\mathrm{C}_{15} \mathrm{H}_{22} \mathrm{~N}_{2} \mathrm{O}_{2}[\mathrm{M}+\mathrm{H}]^{+} 263.1754$ found 263.1744

Hence, $6 q$ was obtained in $89 \%$ yield over two steps. 
$\mathrm{R}^{1} \mathrm{COOH}(7)$

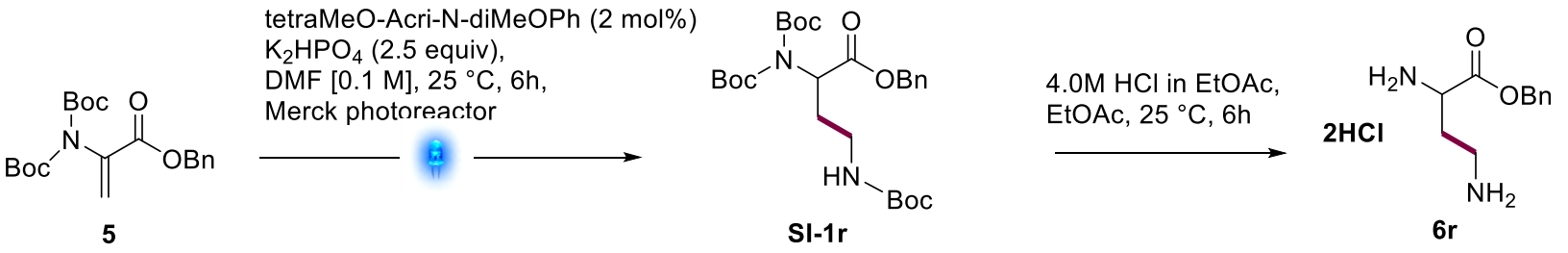

Prepared by Method B using Boc-gly-OH (70 mg, 0.397mmol, 1.5 eq) for 15 hours on PennOC Photoreactor ${ }^{\circledR}$. Obtained $\mathbf{S I}-1 \mathrm{r}$ as yellow oil (125 mg, $0.246 \mathrm{mmol}, 93 \%$ yield).

${ }^{1} \mathrm{H}$ NMR $(500 \mathrm{MHz}, \mathrm{MeOD}) \delta 7.40-7.28(\mathrm{~m}, 5 \mathrm{H}), 5.21-5.09(\mathrm{~m}, 2 \mathrm{H}), 4.98(\mathrm{dd}, J=8.5,5.6 \mathrm{~Hz}, 1 \mathrm{H}), 3.17-3.05(\mathrm{~m}$, $2 \mathrm{H}), 2.33(\mathrm{dd}, J=13.7,6.6 \mathrm{~Hz}, 1 \mathrm{H}), 1.97(\mathrm{dd}, J=14.4,7.6 \mathrm{~Hz}, 1 \mathrm{H}), 1.43(\mathrm{~d}, J=6.3 \mathrm{~Hz}, 27 \mathrm{H})$.

${ }^{13} \mathrm{C}$ NMR (126 MHz, MeOD) $\delta$ 171.9, 158.4, 153.6, 137.2, 129.5, 129.3, 129.2, 84.7, 80.1, 68.0, 57.4, 38.6, 31.3, 28.8, 28.2

HRMS calcd for $\mathrm{C}_{26} \mathrm{H}_{40} \mathrm{~N}_{2} \mathrm{O}_{8} \mathrm{Na}[\mathrm{M}+\mathrm{Na}]^{+} 531.2677$ found 531.2664

SI-1r (100 mg, $0.197 \mathrm{mmol}$ ) was subjected to bisBoc deprotection affording $6 \mathrm{r}$ as white solid $(53.7 \mathrm{mg}, 0.191 \mathrm{mmol}$, $97 \%$ yield).

${ }^{1} \mathrm{H}$ NMR $\left(500 \mathrm{MHz}\right.$, DMSO- $\left.d_{6}\right) \delta 9.15-7.97(\mathrm{~m}, 6 \mathrm{H}), 7.52-7.30(\mathrm{~m}, 5 \mathrm{H}), 5.26(\mathrm{~s}, 2 \mathrm{H}), 4.33(\mathrm{t}, J=6.7 \mathrm{~Hz}, 1 \mathrm{H}), 3.09-$ $2.91(\mathrm{~m}, 2 \mathrm{H}), 2.32-2.08(\mathrm{~m}, 2 \mathrm{H})$.

${ }^{13} \mathrm{C}$ NMR $\left(126 \mathrm{MHz}, \mathrm{DMSO}-d_{6}\right) \delta$ 169.2, $135.5,128.9,128.9,128.7,67.9,50.1,35.7,28.3$.

HRMS calcd for $\mathrm{C}_{11} \mathrm{H}_{17} \mathrm{~N}_{2} \mathrm{O}_{2}[\mathrm{M}+\mathrm{H}]^{+} 209.1285$ found 209.1276

Hence, $6 r$ was obtained in $90 \%$ yield over two steps. 
$\mathbf{R}^{1}$ COOH (7)

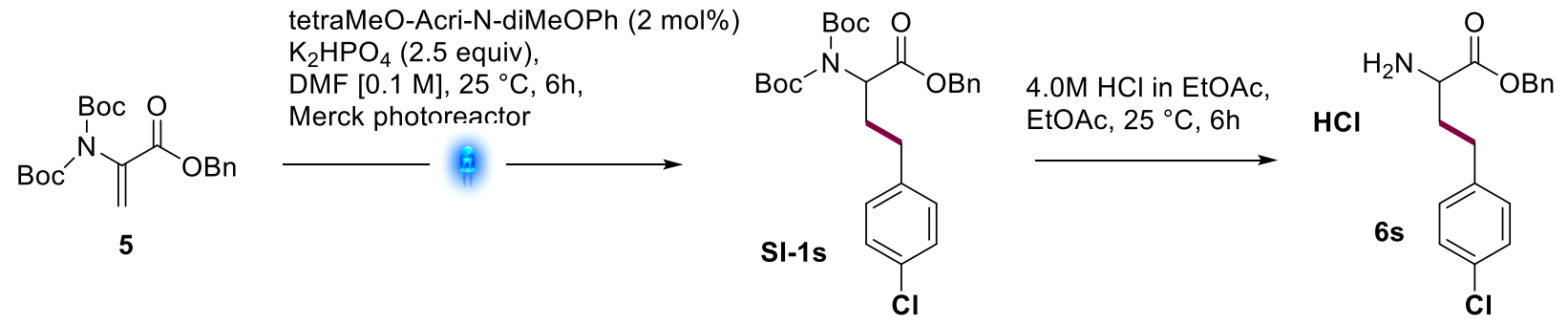

Prepared by Method B using 4-chlorophenylacetic acid $(68 \mathrm{mg}, 0.397 \mathrm{mmol}, 1.5 \mathrm{eq})$ for 10 hours on PennOC Photoreactor ${ }^{\circledast}$.

Obtained SI-1s as yellow oil (105 mg, $0.209 \mathrm{mmol}, 78 \%$ yield).

${ }^{1} \mathrm{H}$ NMR $(500 \mathrm{MHz}, \mathrm{MeOD}) 67.37-7.28(\mathrm{~m}, 5 \mathrm{H}), 7.25(\mathrm{~d}, J=8.4 \mathrm{~Hz}, 2 \mathrm{H}), 7.16(\mathrm{~d}, J=8.4 \mathrm{~Hz}, 2 \mathrm{H}), 5.20-5.03(\mathrm{~m}, 2 \mathrm{H})$, $4.89(\mathrm{dd}, J=9.6,5.2 \mathrm{~Hz}, 1 \mathrm{H}), 2.71-2.61(\mathrm{~m}, 2 \mathrm{H}), 2.48-2.41(\mathrm{~m}, 1 \mathrm{H}), 2.20-2.13(\mathrm{~m}, 1 \mathrm{H}), 1.41(\mathrm{~s}, 18 \mathrm{H})$.

${ }^{13} \mathrm{C}$ NMR $(126 \mathrm{MHz}, \mathrm{MeOD}) \delta 171.9,153.7,141.2,137.2,132.9,131.1,129.6,129.5,129.3,129.2,84.5,67.9,58.9$, $32.8,32.1,28.2$

HRMS calcd for $\mathrm{C}_{27} \mathrm{H}_{34} \mathrm{ClNO}_{6} \mathrm{Na}[\mathrm{M}+\mathrm{Na}]^{+} 526.1972$ found 526.1949

SI-1s (83 mg, $0.165 \mathrm{mmol}$ ) was subjected to bisBoc deprotection affording $6 \mathrm{~s}$ as white solid $(54 \mathrm{mg}, 0.159 \mathrm{mmol}$, $96 \%$ yield).

${ }^{1} \mathrm{H}$ NMR $(500 \mathrm{MHz}, \mathrm{MeOD}) \delta 7.47-7.43(\mathrm{~m}, 2 \mathrm{H}), 7.42-7.36(\mathrm{~m}, 3 \mathrm{H}), 7.26$ (d, $\left.J=8.4 \mathrm{~Hz}, 2 \mathrm{H}\right), 7.11$ (d, $J=8.4 \mathrm{~Hz}$, $2 \mathrm{H}), 5.41-5.18(\mathrm{~m}, 2 \mathrm{H}), 4.09(\mathrm{t}, J=6.2 \mathrm{~Hz}, 1 \mathrm{H}), 2.73(\mathrm{ddd}, J=13.9,10.7,5.7 \mathrm{~Hz}, 1 \mathrm{H}), 2.58(\mathrm{ddd}, J=13.8,10.4,6.4$ $\mathrm{Hz}, 1 \mathrm{H}), 2.22-2.08(\mathrm{~m}, 2 \mathrm{H})$.

${ }^{13} \mathrm{C}$ NMR (126 MHz, MeOD) $\delta$ 170.2, 139.8, 136.4, 133.4, 131.0, 130.0, 129.9, 129.8, 129.7, 69.2, 53.5, 33.3, 31.1.

HRMS calcd for $\mathrm{C}_{17} \mathrm{H}_{18} \mathrm{ClNO}_{2}[\mathrm{M}+\mathrm{H}]^{+} 304.1099$ found 304.1090

Hence, 6 s was obtained in $75 \%$ yield over two steps. 


\section{$\mathbf{R}^{1} \mathrm{COOH}(7)$}

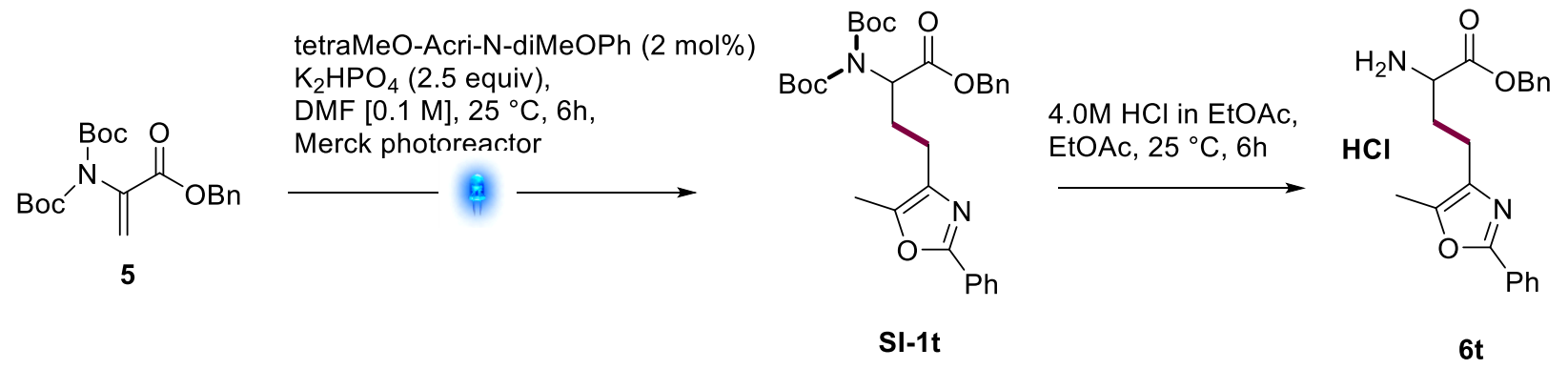

Prepared by Method B using 2-(5-methyl-2-phenyl-1,3-oxazol-4-yl)acetic acid (86 mg, 0.397mmol, 1.5 eq) for 20 hours on PennOC Photoreactor ${ }^{\circledR}$.

Obtained $6 \mathrm{t}$ as yellow oil $(98 \mathrm{mg}, 0.178 \mathrm{mmol}, 67 \%$ yield).

${ }^{1} \mathrm{H}$ NMR $(500 \mathrm{MHz}, \mathrm{MeOD}) \delta 8.01-7.87(\mathrm{~m}, 2 \mathrm{H}), 7.46(\mathrm{dd}, J=5.3,1.8 \mathrm{~Hz}, 3 \mathrm{H}), 7.40-7.24(\mathrm{~m}, 5 \mathrm{H}), 5.23-5.07(\mathrm{~m}$, $2 \mathrm{H}), 4.91(\mathrm{dd}, J=9.0,4.9 \mathrm{~Hz}, 1 \mathrm{H}), 2.58(\mathrm{~d}, J=9.4 \mathrm{~Hz}, 3 \mathrm{H}), 2.29(\mathrm{~s}, 3 \mathrm{H}), 2.28-2.16(\mathrm{~m}, 1 \mathrm{H}), 1.39(\mathrm{~s}, 18 \mathrm{H})$.

${ }^{13} \mathrm{C}$ NMR $(126 \mathrm{MHz}$, MeOD) $\delta$ 171.9, 161.2, 153.7, 145.9, 137.2, 135.7, 131.4, 129.9, 129.5, 129.3, 129.3, 128.6, $126.9,84.5,67.9,58.8,29.4,28.2,23.2,10.0$.

HRMS calcd for $\mathrm{C}_{31} \mathrm{H}_{38} \mathrm{~N}_{2} \mathrm{O}$ - Na $[\mathrm{M}+\mathrm{Na}]^{+} 573.2571$ found 573.2545

SI-1t ( $85 \mathrm{mg}, 0.154 \mathrm{mmol}$ ) was subjected to bisBoc deprotection affording $6 \mathrm{t}$ as white solid ( $58 \mathrm{mg}, 0.150 \mathrm{mmol}, 97 \%$ yield).

${ }^{1} \mathrm{H}$ NMR $\left(500 \mathrm{MHz}\right.$, DMSO- $\left.d_{6}\right) \delta 8.82-8.71(\mathrm{~m}, 3 \mathrm{H}), 7.90-7.89(\mathrm{~m}, 2 \mathrm{H}), 7.52-7.48(\mathrm{~m}, 3 \mathrm{H}), 7.44(\mathrm{app} \mathrm{d}, J=6.7 \mathrm{~Hz}$, $2 \mathrm{H}), 7.40-7.34(\mathrm{~m}, 3 \mathrm{H}), 5.24(\mathrm{~s}, 2 \mathrm{H}), 4.17(\mathrm{~d}, J=5.6 \mathrm{~Hz}, 1 \mathrm{H}), 2.66(\mathrm{dd}, J=15.1,7.1 \mathrm{~Hz}, 1 \mathrm{H}), 2.55(\mathrm{dt}, J=15.1,7.9 \mathrm{~Hz}$, $1 \mathrm{H}), 2.28(\mathrm{~s}, 3 \mathrm{H}), 2.16(\mathrm{q}, J=7.5 \mathrm{~Hz}, 2 \mathrm{H})$.

${ }^{13} \mathrm{C}$ NMR $(126 \mathrm{MHz}$, DMSO-d 6 ) $\delta 169.70,158.97,144.63,135.61,134.62,130.62,129.55,128.97,128.89,128.76$, $127.52,125.90,67.63,52.04,29.91,21.14,10.23$.

HRMS calcd for $\mathrm{C}_{21} \mathrm{H}_{23} \mathrm{~N}_{2} \mathrm{O}_{3}[\mathrm{M}+\mathrm{H}]^{+} 351.1703$ found 351.1689

Hence, 6 t was obtained in $65 \%$ yield over two steps. 


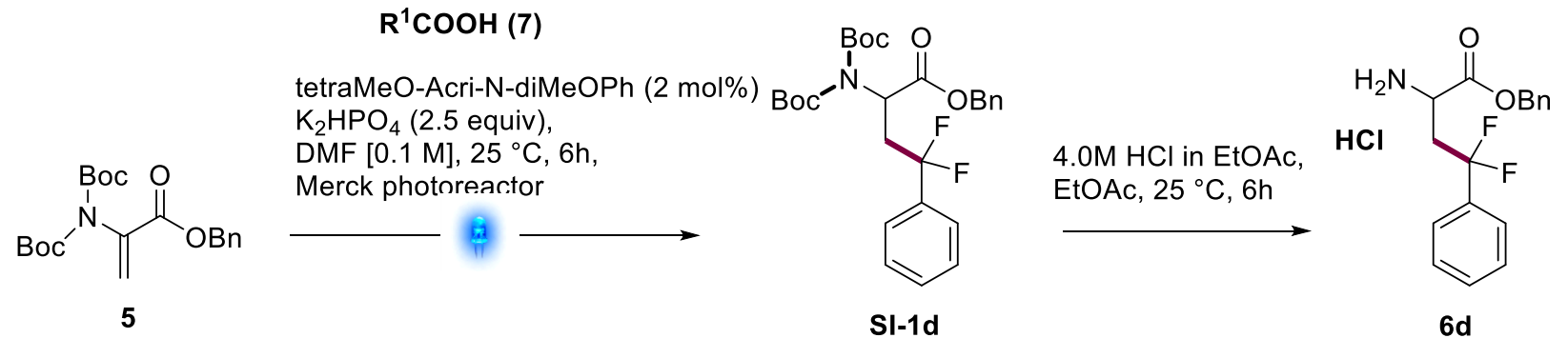

Prepared by Method B using 2,2-difluoro-2-phenylacetic acid ( $68 \mathrm{mg}, 0.397 \mathrm{mmol}, 1.5$ eq) for 48 hours on PennOC Photoreactor ${ }^{\circledR}$.

Obtained SI-1d as yellow oil ( $37 \mathrm{mg}, 0.073 \mathrm{mmol}, 28 \%$ yield).

${ }^{1} \mathrm{H} \mathrm{NMR}(500 \mathrm{MHz}, \mathrm{MeOD}) \delta 7.53-7.41(\mathrm{~m}, 5 \mathrm{H}), 7.32(\mathrm{~d}, J=3.0 \mathrm{~Hz}, 5 \mathrm{H}), 5.26(\mathrm{dd}, J=9.8,2.9 \mathrm{~Hz}, 1 \mathrm{H}), 5.16(\mathrm{~d}, J=$ $12.4 \mathrm{~Hz}, 1 \mathrm{H}), 5.12(\mathrm{~d}, J=12.4 \mathrm{~Hz}, 1 \mathrm{H}), 3.08(\mathrm{qd}, J=15.8,2.9 \mathrm{~Hz}, 1 \mathrm{H}), 2.84(\mathrm{qd}, J=15.8,9.9 \mathrm{~Hz}, 1 \mathrm{H}), 1.39(\mathrm{~s}, 18 \mathrm{H})$.

${ }^{13} \mathrm{C}$ NMR (126 MHz, MeOD) $\delta$ 171.0, 153.1, $137.8(\mathrm{t}, J=26.0 \mathrm{~Hz}), 136.9,131.2,129.8,129.6,129.4,129.2,126.0$ (t, $J$ $=6.3 \mathrm{~Hz}), 123.3(\mathrm{t}, J=242.2 \mathrm{~Hz}), 84.7,68.5,54.9,39.4(\mathrm{t}, J=27.9 \mathrm{~Hz}), 28.2$.

HRMS calcd for $\mathrm{C}_{27} \mathrm{H}_{33} \mathrm{~F}_{2} \mathrm{NO}_{6} \mathrm{Na}[\mathrm{M}+\mathrm{Na}]^{+} 528.2174$ found 528.2158

SI-1d (19 mg, $0.038 \mathrm{mmol}$ ) was subjected to bisBoc deprotection affording $6 \mathbf{d}$ as white solid $(13 \mathrm{mg}, 0.037 \mathrm{mmol}$, $97 \%$ yield).

${ }^{1} \mathrm{H}$ NMR $\left(500 \mathrm{MHz}, \mathrm{DMSO}-d_{6}\right) \delta 8.76(\mathrm{~s}, 3 \mathrm{H}), 7.59-7.48(\mathrm{~m}, 5 \mathrm{H}), 7.46-7.35(\mathrm{~m}, 5 \mathrm{H}), 5.22(\mathrm{~d}, J=12.4 \mathrm{~Hz}, 1 \mathrm{H}), 5.11$ (d, $J=12.4 \mathrm{~Hz}, 1 \mathrm{H}), 4.28(\mathrm{t}, J=5.9 \mathrm{~Hz}, 1 \mathrm{H}), 2.90$ (ddd, $J=20.6,14.2,5.8 \mathrm{~Hz}, 2 \mathrm{H}$ ).

${ }^{13} \mathrm{C}$ NMR $\left(126 \mathrm{MHz}\right.$, DMSO- $\left.d_{6}\right) \delta 168.7,135.54(\mathrm{t}, J=25.5 \mathrm{~Hz}), 135.3,131.2,129.4,128.9,128.9,128.8,125.24(\mathrm{t}, J=$ $6.1 \mathrm{~Hz}), 121.9(\mathrm{t}, J=242.6 \mathrm{~Hz}), 67.9,48.2,38.9(\mathrm{t}, J=27.3 \mathrm{~Hz})$.

HRMS calcd for $\mathrm{C}_{17} \mathrm{H}_{17} \mathrm{~F}_{2} \mathrm{NO}_{2} \mathrm{Na}[\mathrm{M}+\mathrm{Na}]^{+} 328.1120$ found 328.1122

\section{Hence, $\mathbf{6 d}$ was obtained in $\mathbf{2 7 \%}$ yield over two steps.}




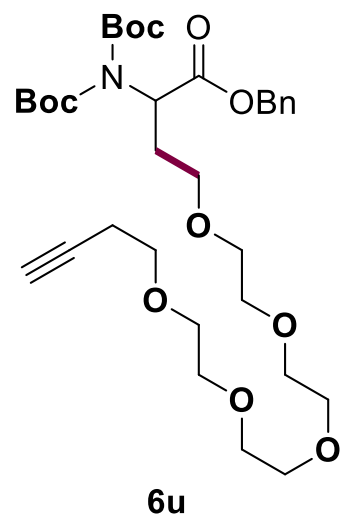

Prepared by Method B using 7u (121 mg, 0.397mmol, 1.5 eq) for 48 hours on PennOC Photoreactor ${ }^{\circledR}$.

Obtained $6 \mathrm{u}$ as colorless oil (35 $\mathrm{mg}, 0.055 \mathrm{mmol}, 21 \%$ yield).

${ }^{1} \mathrm{H}$ NMR (500 MHz, MeOD) $\delta 7.60-6.91(\mathrm{~m}, 5 \mathrm{H}), 5.41-5.07(\mathrm{~m}, 2 \mathrm{H}), 4.94(\mathrm{dd}, J=9.9,5.1 \mathrm{~Hz}, 1 \mathrm{H}), 4.18(\mathrm{~d}, J=2.4 \mathrm{~Hz}$, $2 \mathrm{H}), 3.64(\mathrm{dd}, J=15.4,2.3 \mathrm{~Hz}, 16 \mathrm{H}), 3.50(\mathrm{t}, J=6.3 \mathrm{~Hz}, 2 \mathrm{H}), 2.84(\mathrm{t}, J=2.3 \mathrm{~Hz}, 1 \mathrm{H}), 2.33-2.11(\mathrm{~m}, 1 \mathrm{H}), 2.05-1.87$ $(\mathrm{m}, 1 \mathrm{H}), 1.60(\mathrm{dtd}, J=12.8,7.5,6.5,2.9 \mathrm{~Hz}, 2 \mathrm{H}), 1.44(\mathrm{~s}, 18 \mathrm{H})$;

${ }^{13} \mathrm{C}$ NMR (126 MHz, MeOD) $\delta$ 172.0, 153.8, 137.3, 129.6, 129.3, 129.2, 84.5, 80.6, 75.9, 71.60, 71.57, 71.55, 71.5, $71.4,71.2,70.1,67.9,59.3,59.0,28.2,27.4,27.3$.

HRMS calcd for $\mathrm{C}_{33} \mathrm{H}_{51} \mathrm{NO}_{11} \mathrm{Na}[\mathrm{M}+\mathrm{Na}]^{+} 660.3354$ found 660.3361

D. Synthesis of 6 a on $1 \mathrm{mmol}$ scale via batch conditions 


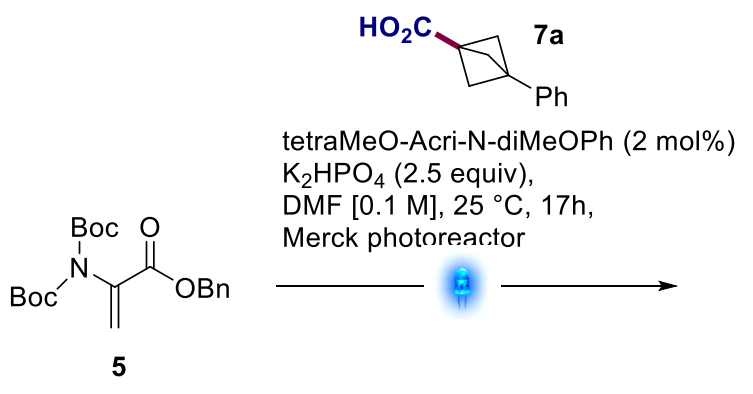

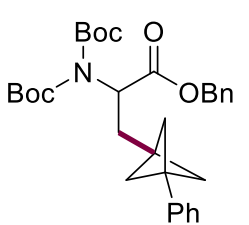

SI-1a

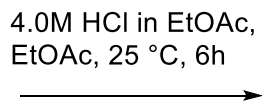

EtOAc, $25^{\circ} \mathrm{C}, 6 \mathrm{~h}$

$\mathrm{HCl}$

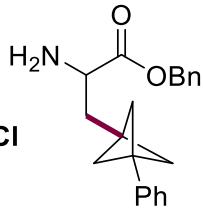

$6 a$

In a clear scintillation vial were added benzyl 2-(bis(tert-butaloxycarbonyl)amino)acrylate (5) (380 mg, $1.0 \mathrm{mmol}$, 1.0 equiv), carboxylic acid 7 ( $284 \mathrm{mg}, 1.51 \mathrm{mmol}, 1.5$ equiv), and tetraMeO-Acri- $\mathrm{N}$-diMeoPh (13 mg, $0.02 \mathrm{mmol}, 0.02$ equiv). DMF (0.13 M) was added to the vials followed by potassium phosphate dibasic (526 $\mathrm{mg}, 3.02 \mathrm{mmol}, 3.0$ equiv). Vial was sealed and then set on the PennOC Photoreactor ${ }^{\circledR}$ (wavelength: $450 \mathrm{~nm}$; LED intensity: 100\%; fan speed: $5000 \mathrm{rpm}$; stir: $1000 \mathrm{rpm})$ for $17 \mathrm{~h}$. After the reaction was complete, DMSO (1mL) was added to the mixture, which was filtered and the filtrate purified by reverse phase (C-18) HPLC (30 x $250 \mathrm{~mm}$, Waters SunFire ${ }^{\circledR}$ OBD ${ }^{\mathrm{TM}} 10$ $\mathrm{mM}$ ) eluting with a gradient of $5-95 \%$ Acetonitrile/Water $+0.1 \%$ TFA over 15 minutes at $43 \mathrm{ml} / \mathrm{min}$. The fractions that contained product were combined, neutralized with $2 \mathrm{ml} \mathrm{NH} \mathrm{H}_{4} \mathrm{OH}$, and concentrated. The residue was diluted with sat $\mathrm{NaHCO}_{3}$ and than extracted with $\mathrm{CH}_{2} \mathrm{Cl}_{2}$. The organic portion was separated, washed with brine, dried over anhydrous $\mathrm{MgSO}$, filtered, and the volatiles were removed under reduced pressure to provide bisBoc adduct $\mathbf{S I - 1 a}$ as a colorless oil (325 mg, $0.62 \mathrm{mmol}, 62 \%)$.

${ }^{1} \mathrm{H}$ NMR (500 MHz, MeOD) $\delta 7.39-7.29(\mathrm{~m}, 5 \mathrm{H}), 7.25(\mathrm{t}, J=7.4 \mathrm{~Hz}, 2 \mathrm{H}), 7.19-7.12(\mathrm{~m}, 3 \mathrm{H}), 5.17(\mathrm{~d}, J=12.4 \mathrm{~Hz}$, $1 \mathrm{H}), 5.13(\mathrm{~d}, J=12.4 \mathrm{~Hz}, 1 \mathrm{H}), 5.03(\mathrm{dd}, J=10.4,4.4 \mathrm{~Hz}, 1 \mathrm{H}), 2.35$ (dd, $J=15.1,4.4 \mathrm{~Hz}, 1 \mathrm{H}$ ), 2.19 (dd, $J=15.1,10.4$ $\mathrm{Hz}, 1 \mathrm{H}), 1.92(\mathrm{~s}, 6 \mathrm{H}), 1.45(\mathrm{~s}, 18 \mathrm{H})$.

${ }^{13} \mathrm{C}$ NMR $(126 \mathrm{MHz}, \mathrm{MeOD}) \delta 172.1,153.6,142.2,137.2,129.5,129.3,129.2,129.1,127.3,126.8,84.5,68.0,58.1$, $53.6,43.1,37.7,32.1,28.3$.

HRMS calcd for $\mathrm{C}_{31} \mathrm{H}_{39} \mathrm{NO}_{6}[\mathrm{M}+\mathrm{H}]^{+} 522.2850$ found 522.2833

\section{Synthesis of $\alpha$-amino benzyl ester hydrochloride (6a):}

SI-1a (325 mg, $0.62 \mathrm{mmol}, 1.0$ equiv) was treated with $8.0 \mathrm{ml}$ of saturated $\mathrm{HCl}$ in EtOAc. The mixture was stirred overnight. After the reaction was complete, volatiles were removed under reduced pressure. Added EtOAc (5 ml), stirred 5 minutes, and then the volatiles were removed under reduce pressure to provide desired product $6 \mathbf{a}$ as a white solid (210 $\mathrm{mg}, 94 \%)$.

${ }^{1} \mathrm{H}$ NMR (500 MHz, MeOD) $\delta 7.46(\mathrm{~d}, J=6.5 \mathrm{~Hz}, 2 \mathrm{H}), 7.43-7.35(\mathrm{~m}, 3 \mathrm{H}), 7.25(\mathrm{t}, J=7.4 \mathrm{~Hz}, 2 \mathrm{H}), 7.17(\mathrm{t}, J=7.4 \mathrm{~Hz}$, $1 \mathrm{H}), 7.14-7.10(\mathrm{~m}, 2 \mathrm{H}), 5.31(\mathrm{~d}, J=3.1 \mathrm{~Hz}, 2 \mathrm{H}), 4.11(\mathrm{dd}, J=6.8,5.2 \mathrm{~Hz}, 1 \mathrm{H}), 2.30-2.08(\mathrm{~m}, 2 \mathrm{H}), 1.94(\mathrm{~s}, 6 \mathrm{H})$.

${ }^{13} \mathrm{C}$ NMR (126 MHz, MeOD) $\delta$ 170.5, 141.5, 136.2, 130.2, 129.9, 129.8, 129.2, 127.6, 126.8, 69.4, 53.6, 52.7, 43.3, 36.3, 33.7.

HRMS calcd for $\mathrm{C}_{21} \mathrm{H}_{23} \mathrm{NO}_{2}[\mathrm{M}+\mathrm{H}]^{+} 322.1802$ found 322.1790 

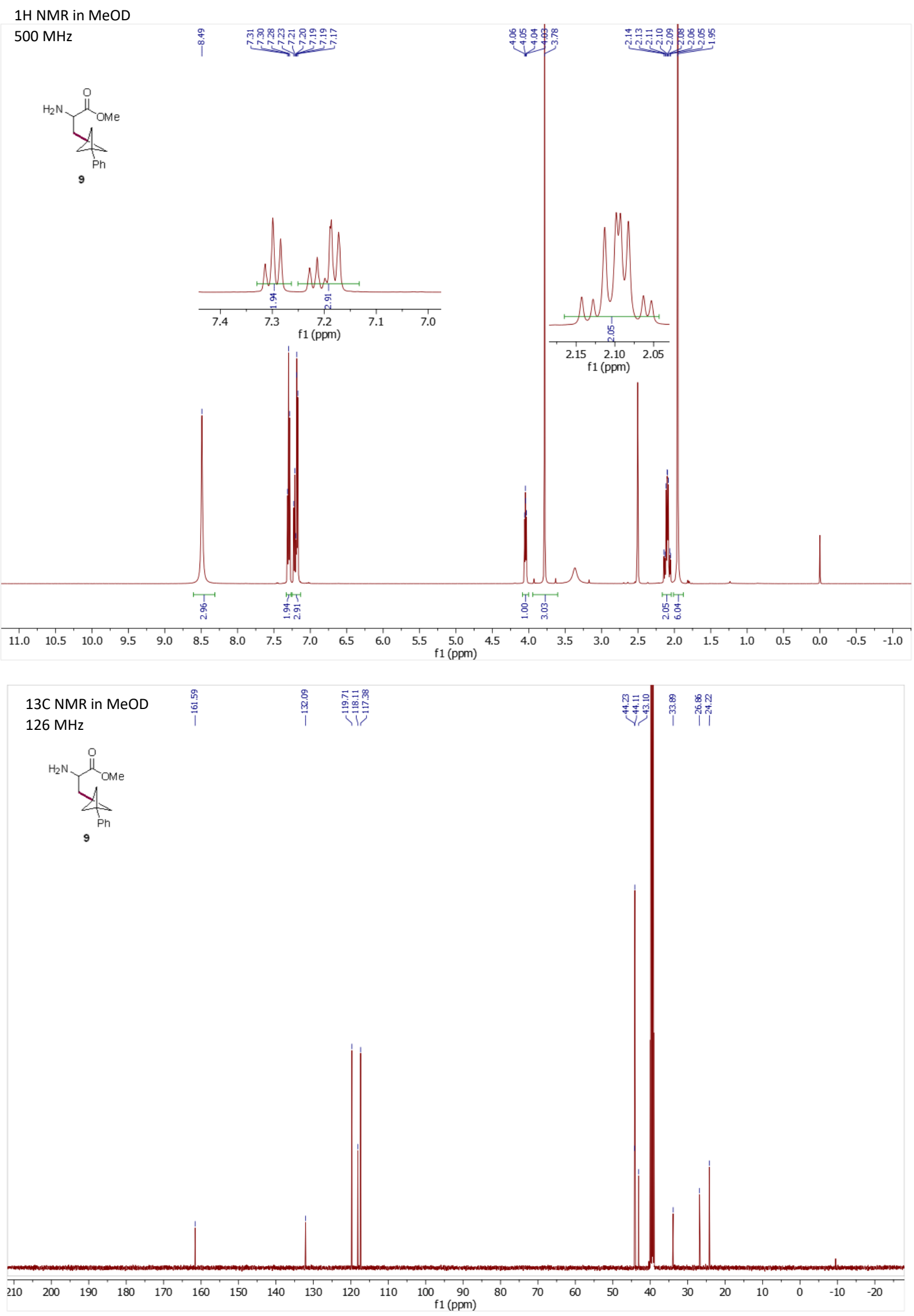

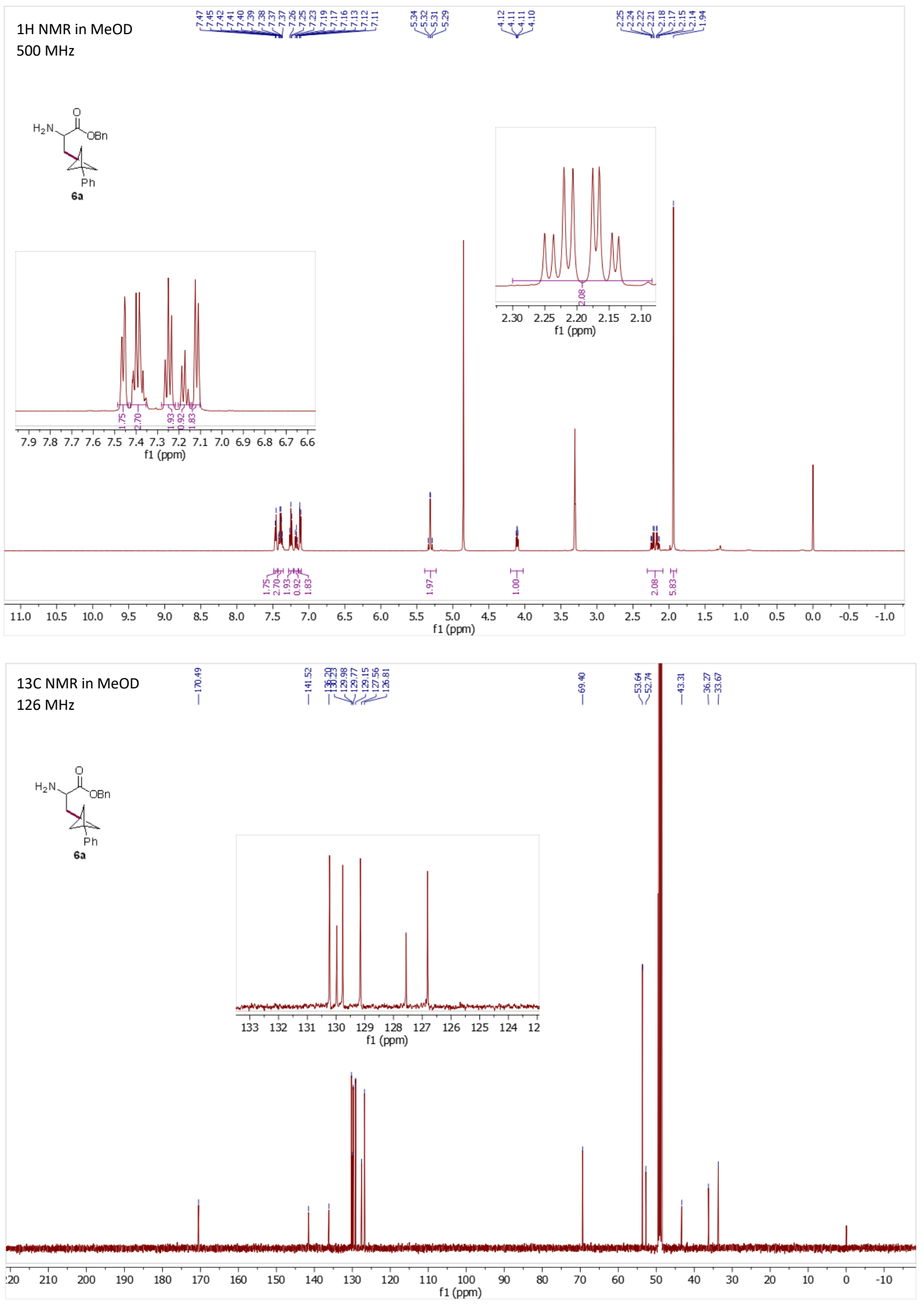
<smiles>NC(CC1(F)CCCCC1)C(=O)O</smiles>
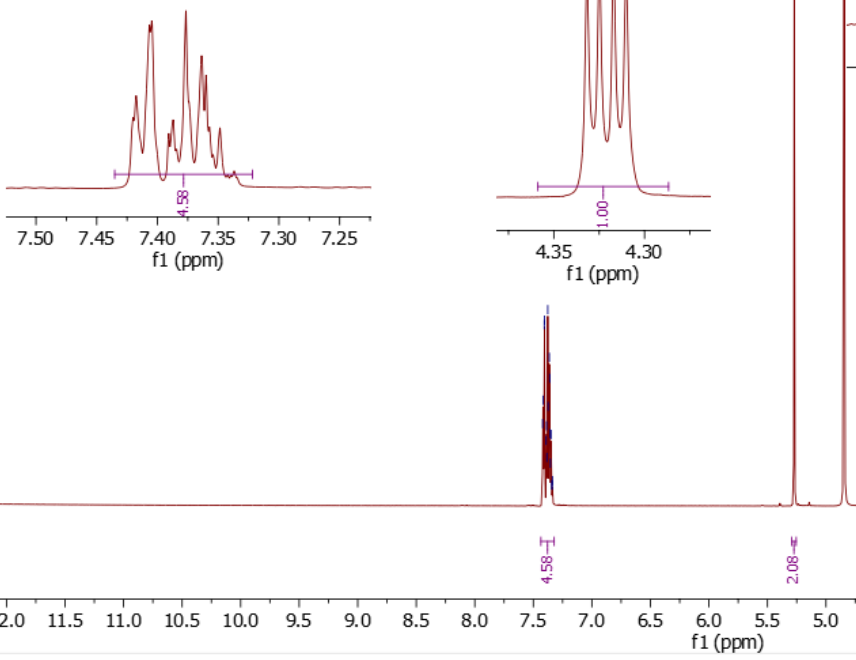

13C NMR in MeOD

$126 \mathrm{MHz}$<smiles>NC(C(=O)O)C1(F)C[C@@H]2CCCC[C@@H]21</smiles>

6b

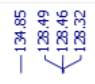

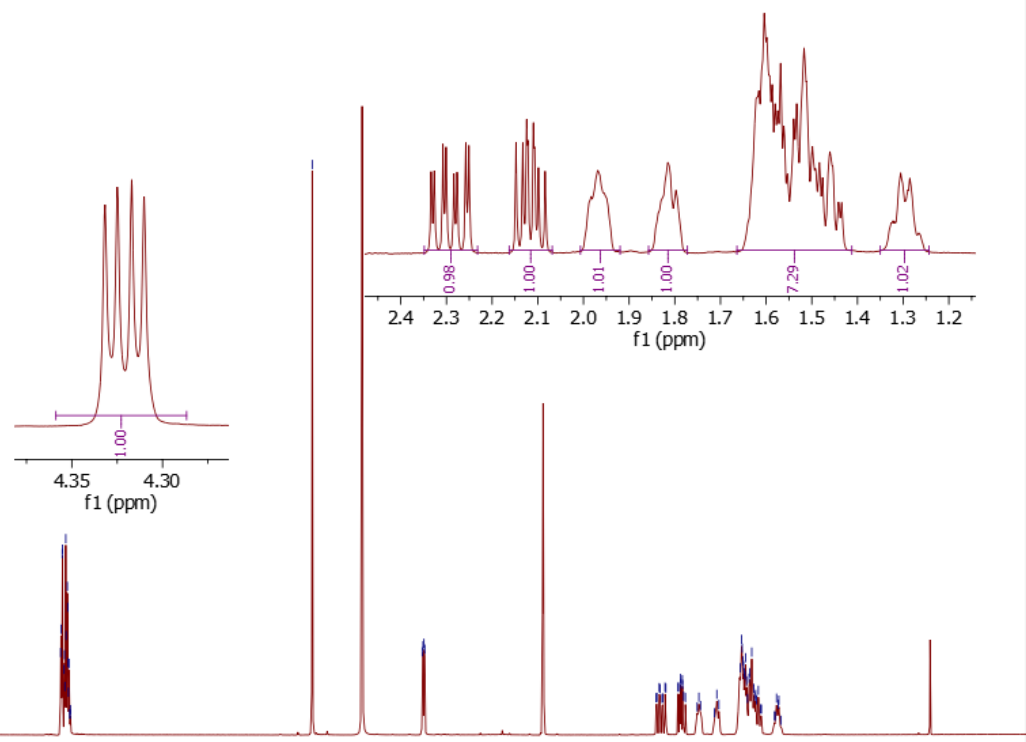

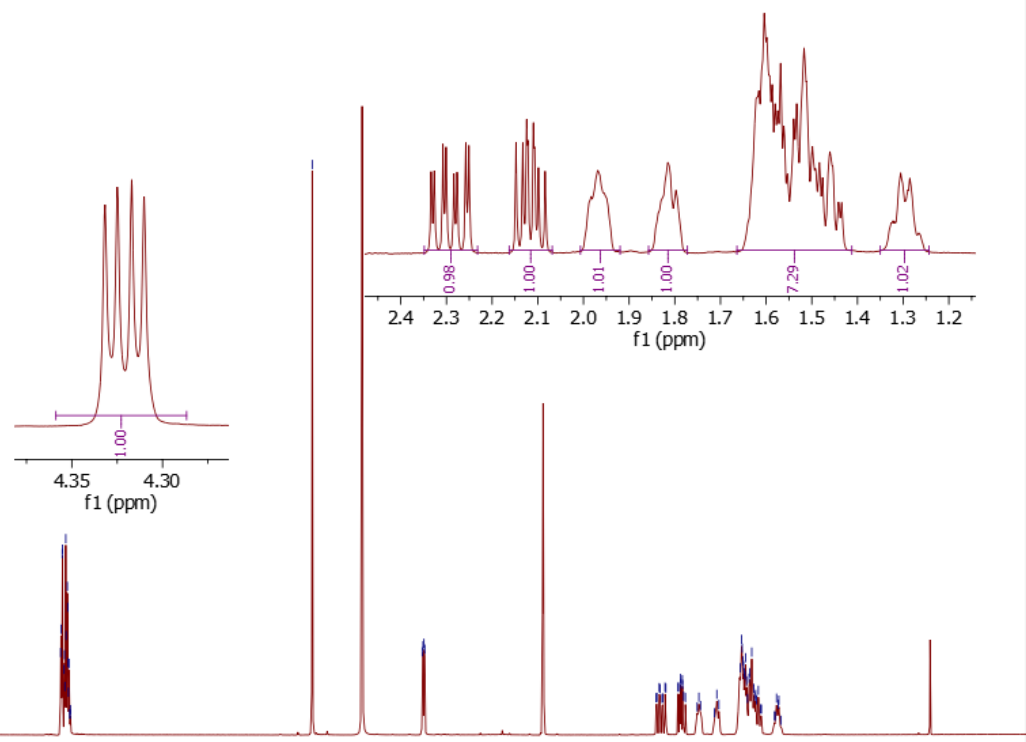

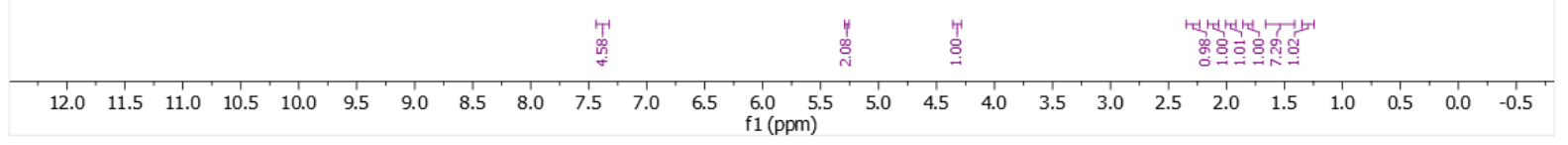

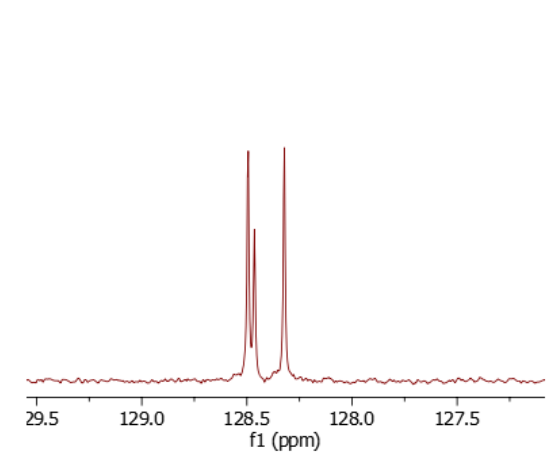

量宫
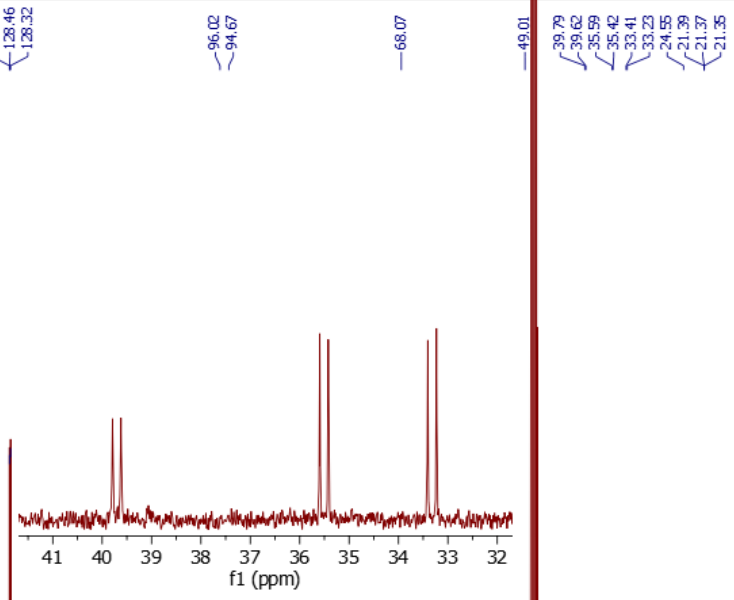

$\begin{array}{llllllllllllllllllllllllll}1 & 220 & 210 & 200 & 190 & 180 & 170 & 160 & 150 & 140 & 130 & 120 & 110 & 100 & 90 & 80 & 70 & 60 & 50 & 40 & 30 & 20 & 10\end{array}$ 


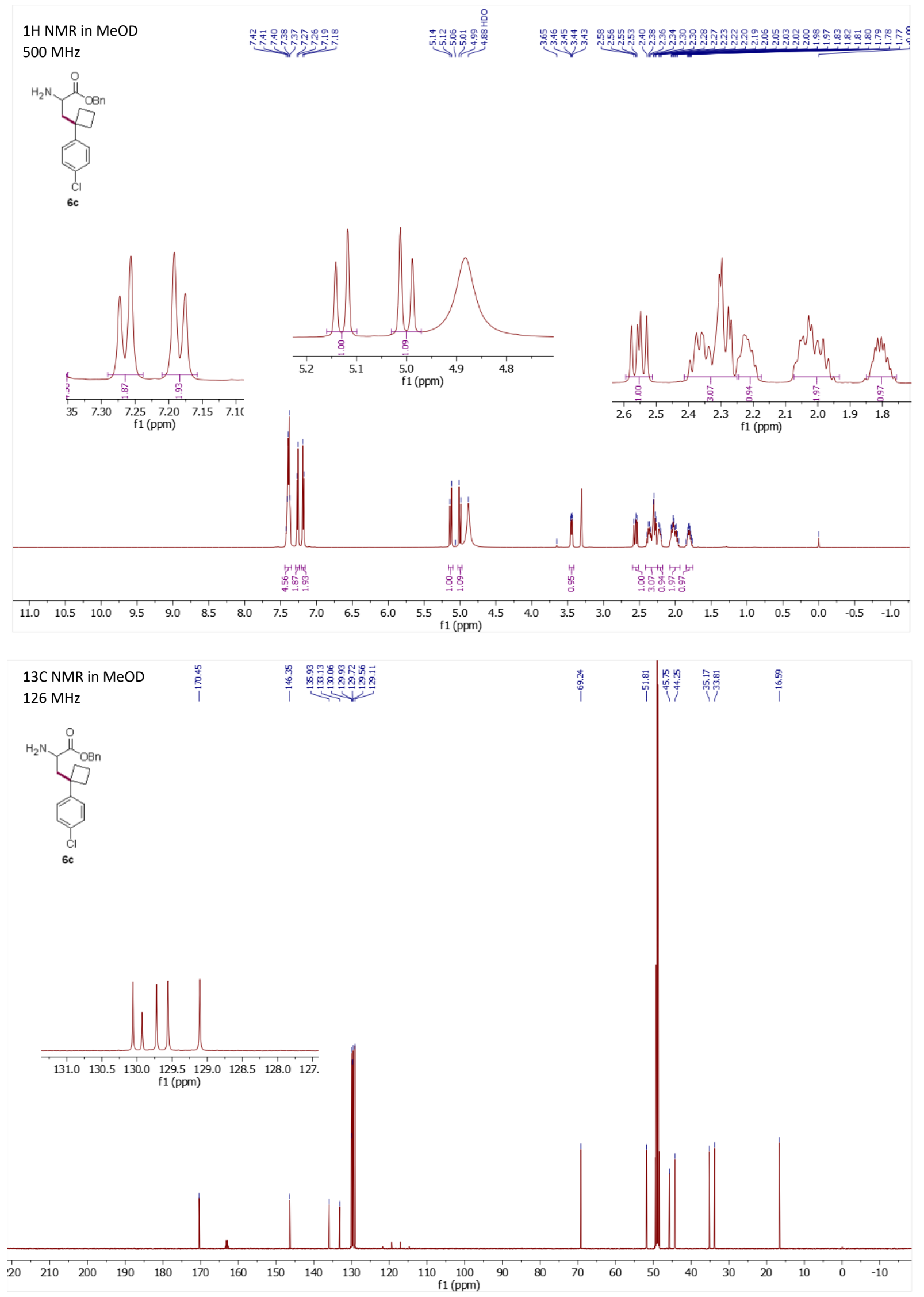



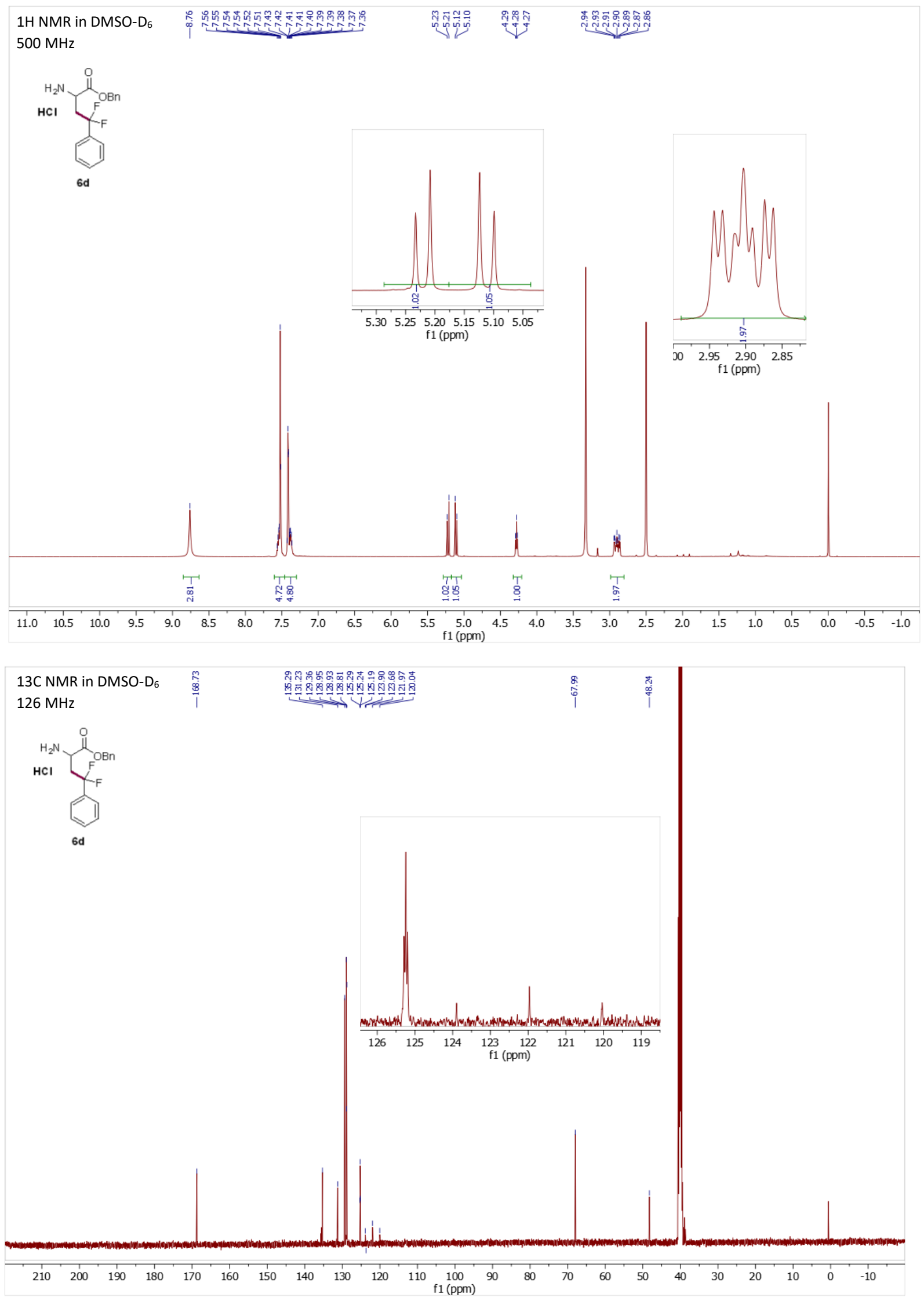


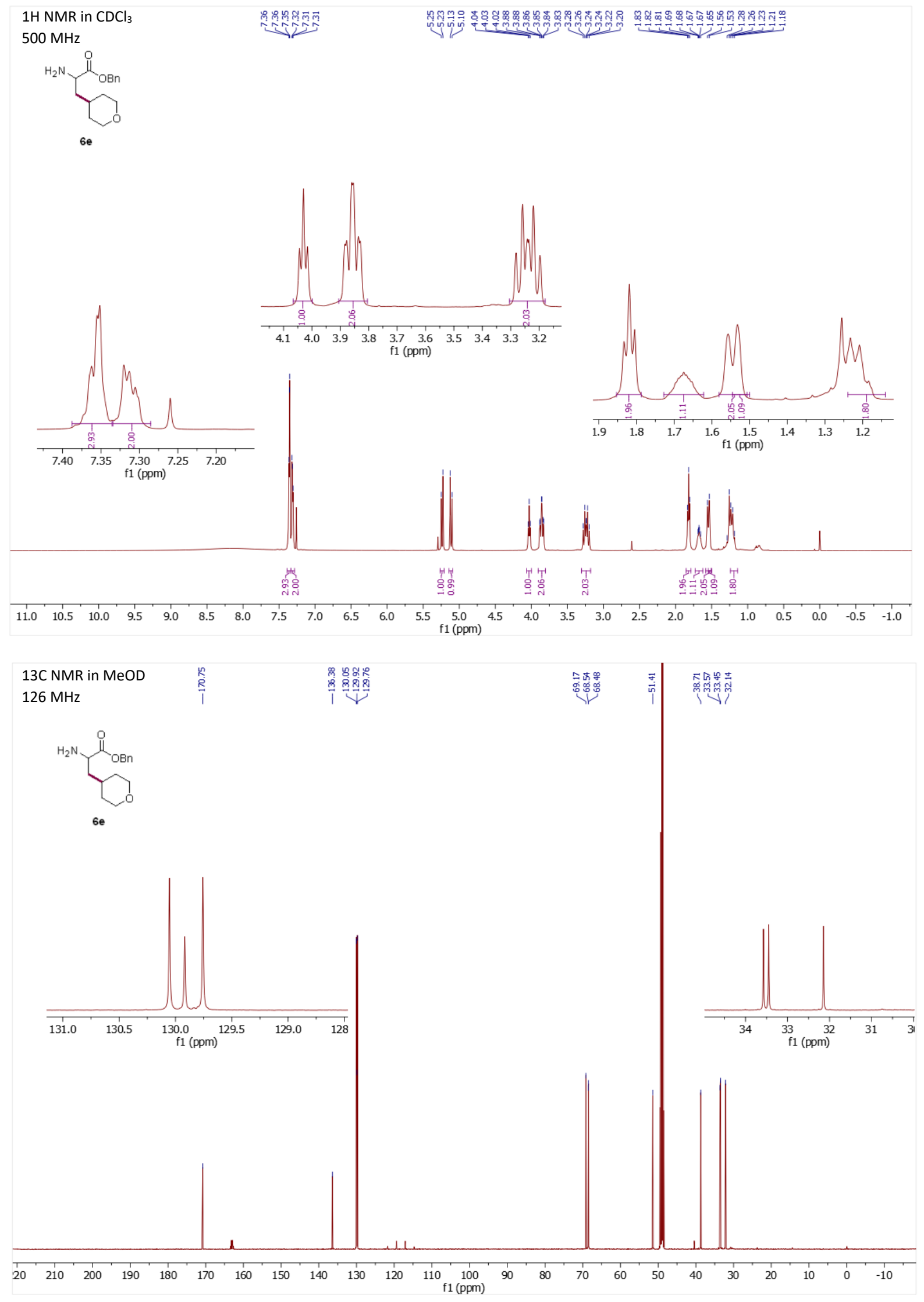




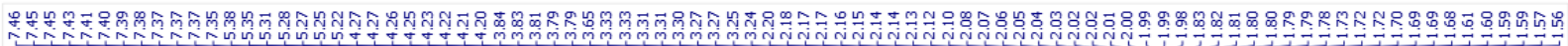
$1 \mathrm{H} N \mathrm{NMR}$ in MeOD

$600 \mathrm{MHz}$<smiles>NC(CC1CCC(F)(F)C(Br)C1)C(=O)C(=O)c1ccccc1</smiles>
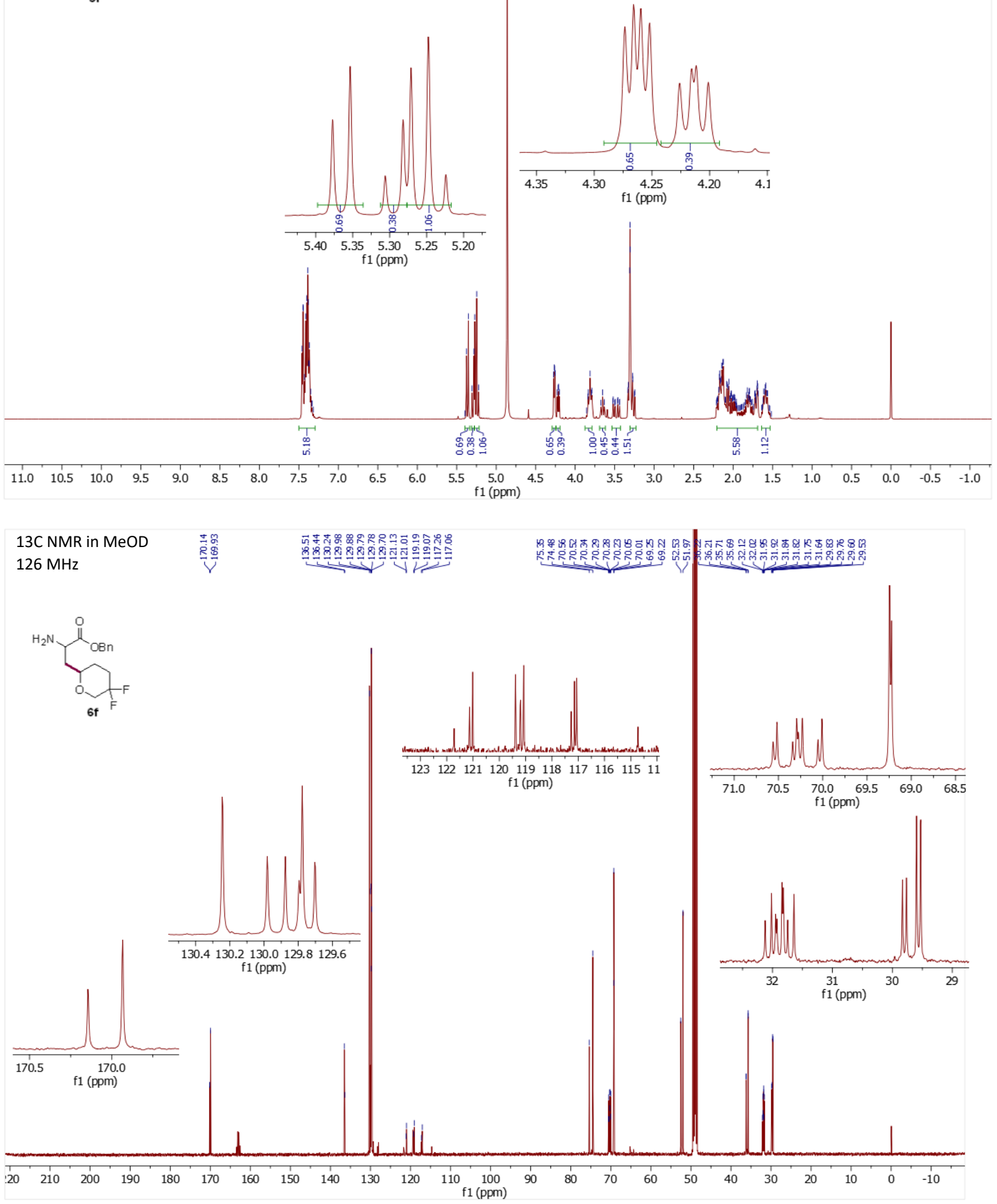


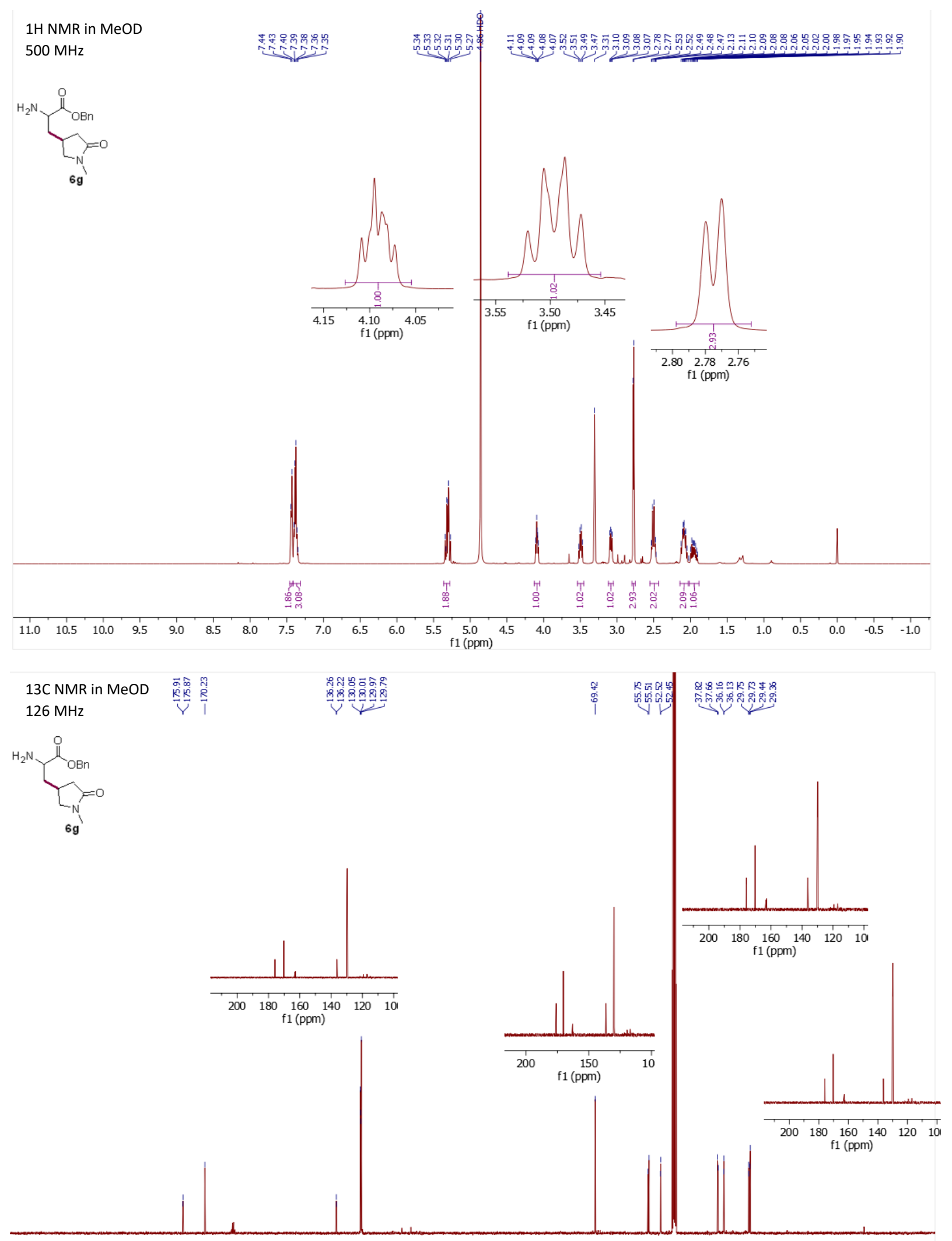

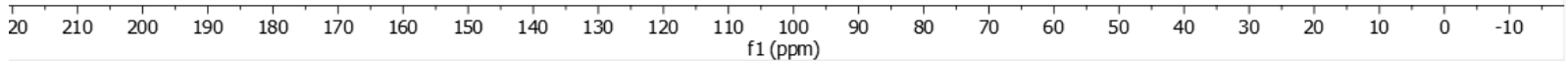




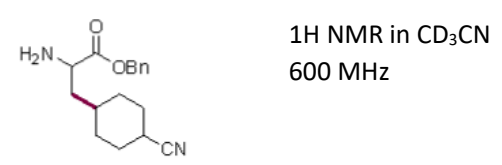

$6 \mathrm{~h}$

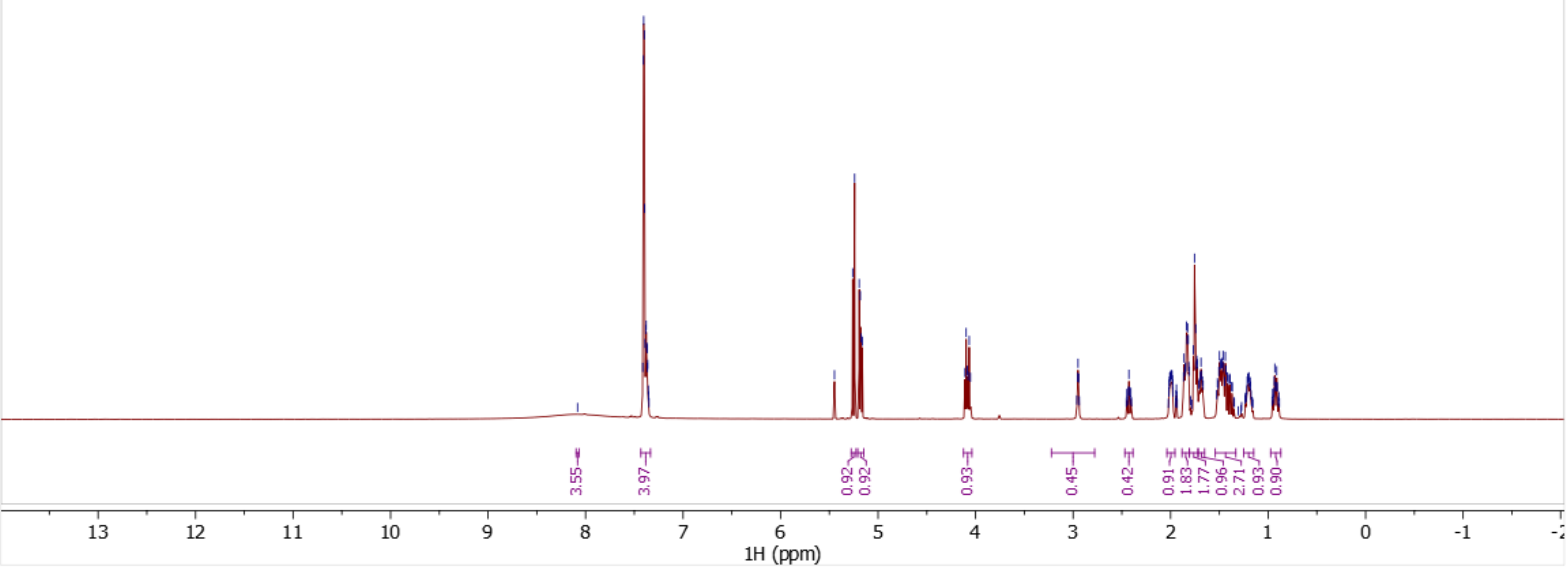

$13 \mathrm{C} N \mathrm{NM}$ in $\mathrm{CD}_{3} \mathrm{CN}$ $151 \mathrm{MHz}$

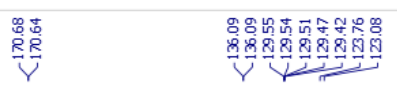
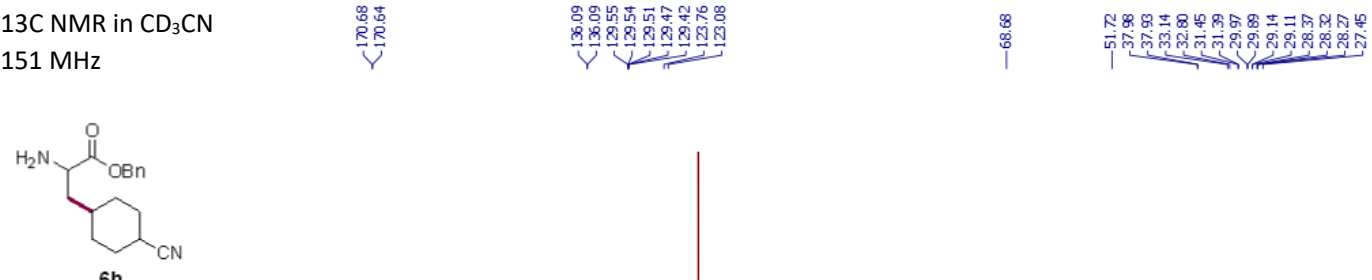

6h

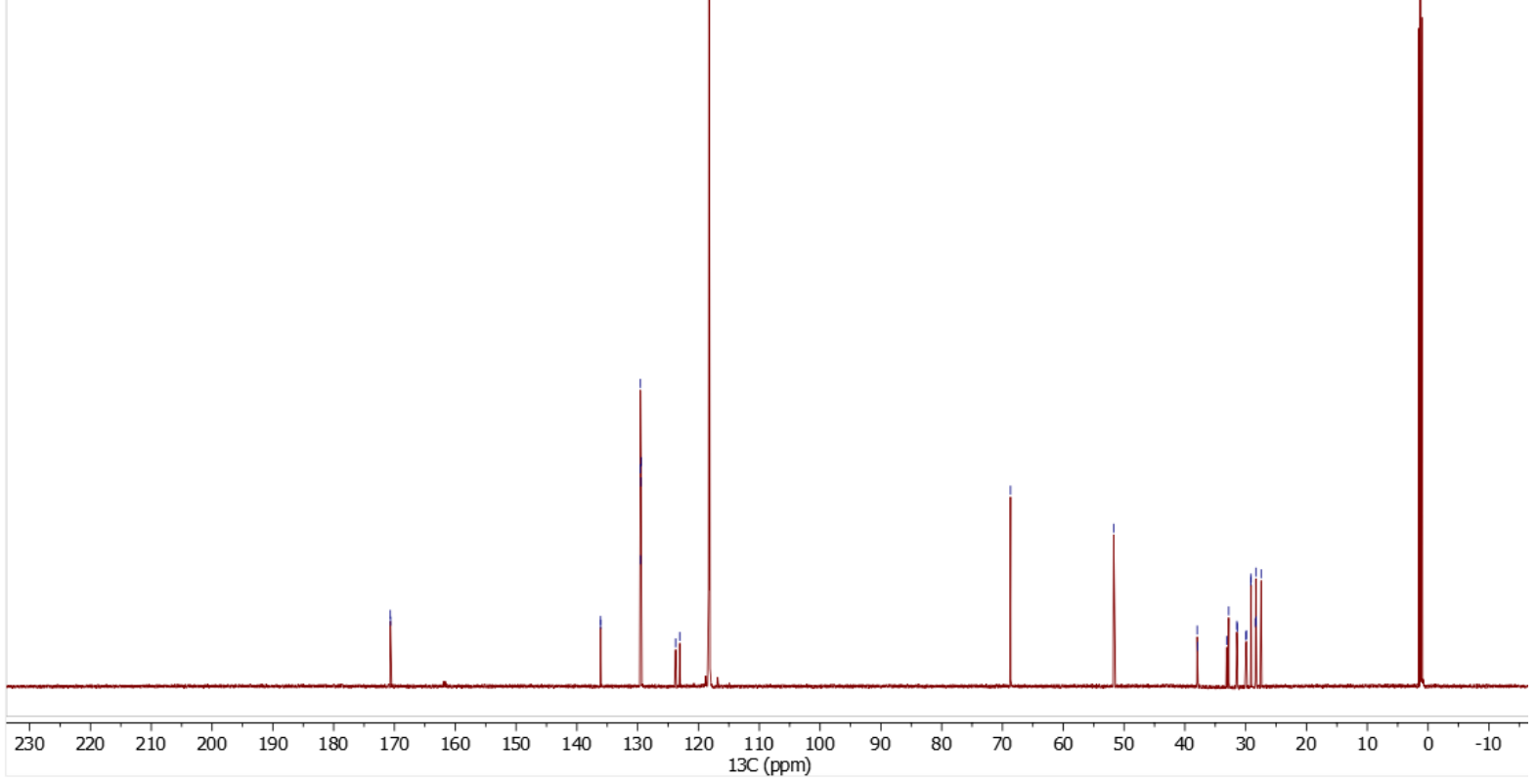


모

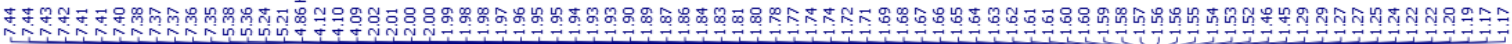

$1 \mathrm{H}$ NMR in MeOD

$500 \mathrm{MHz}$
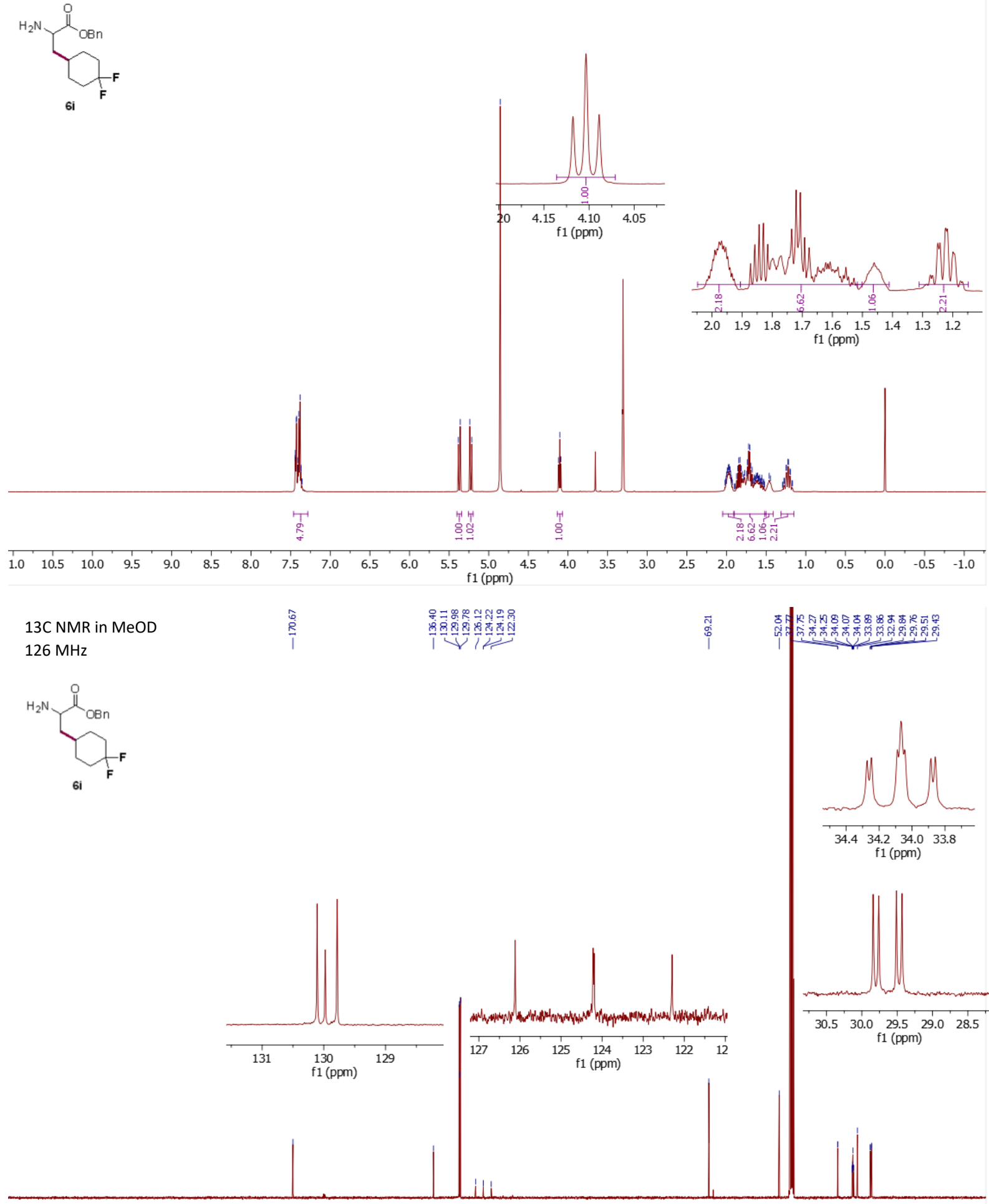

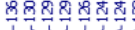

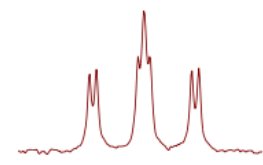

$\begin{array}{llllll}34.4 & 34.2 & 34.0 & 33.8\end{array}$ f1 (ppm)

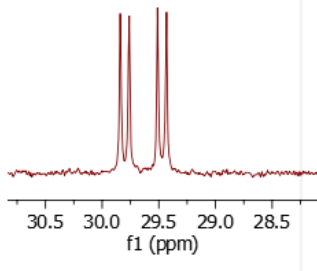

\begin{tabular}{llllllllllllllllllllllllllll}
\hline 10 & 230 & 220 & 210 & 200 & 190 & 180 & 170 & 160 & 150 & 140 & 130 & 120 & 110 & 100 & 90 & 80 & 70 & 60 & 50 & 40 & 30 & 20 & 10
\end{tabular} 

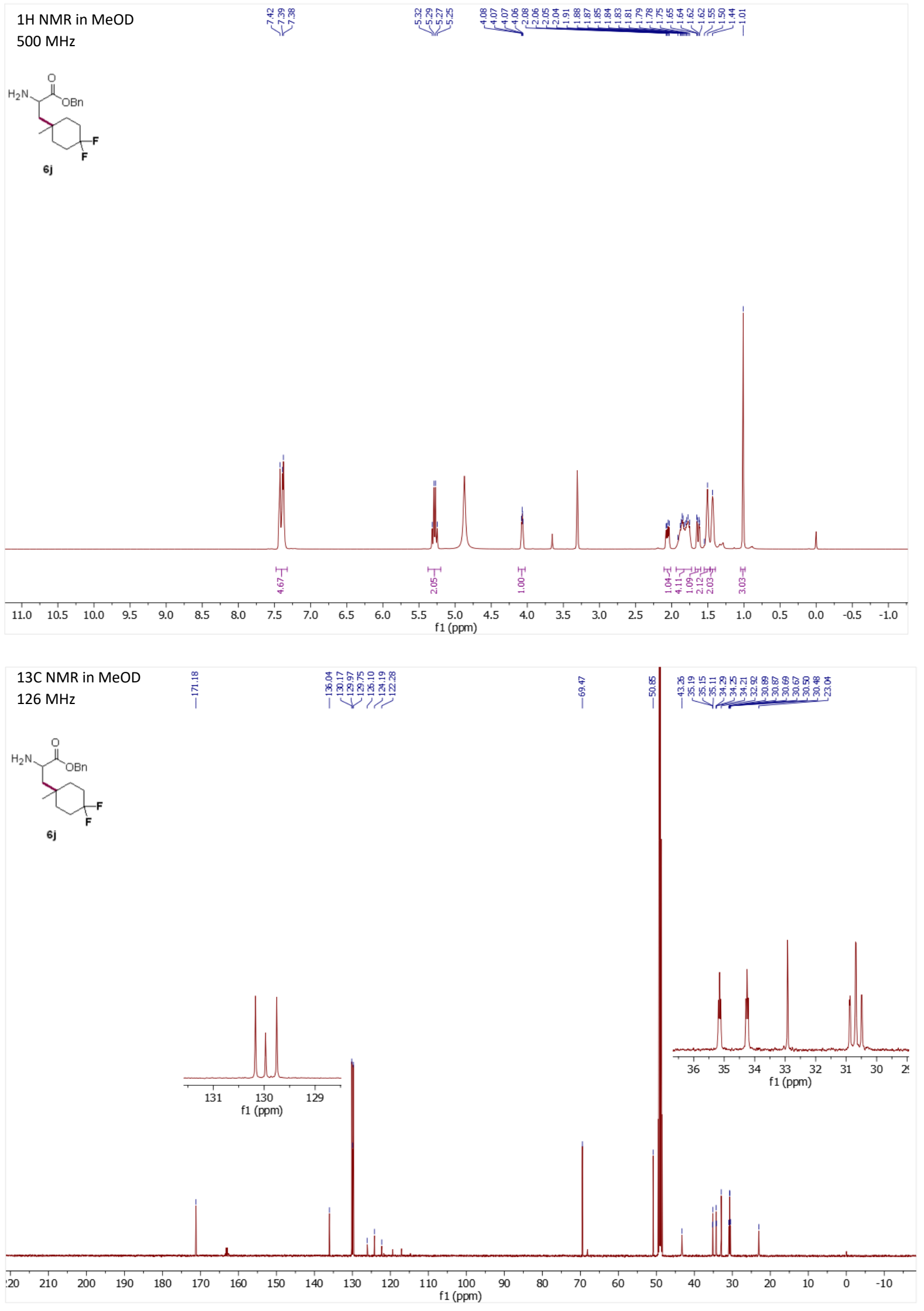

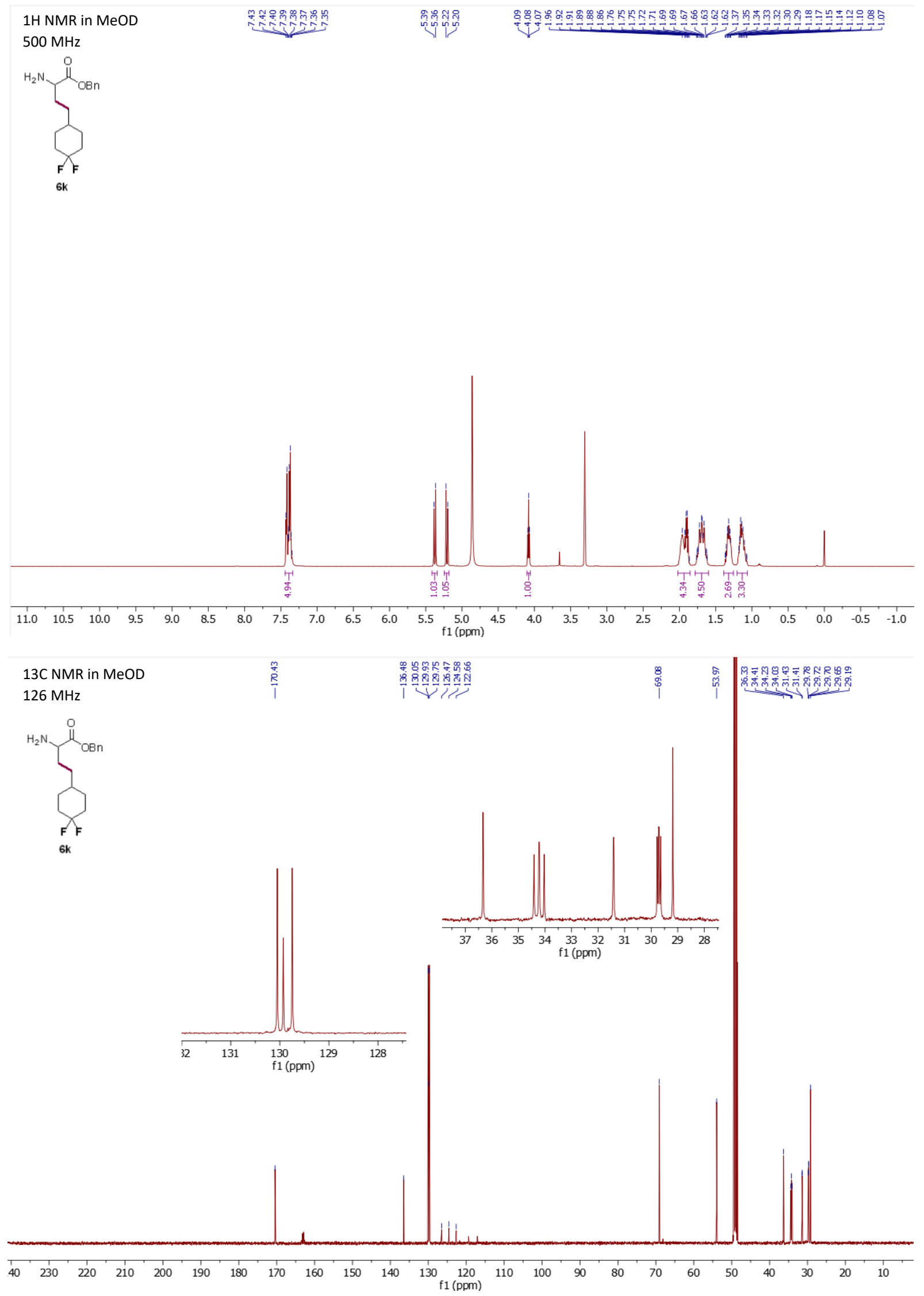


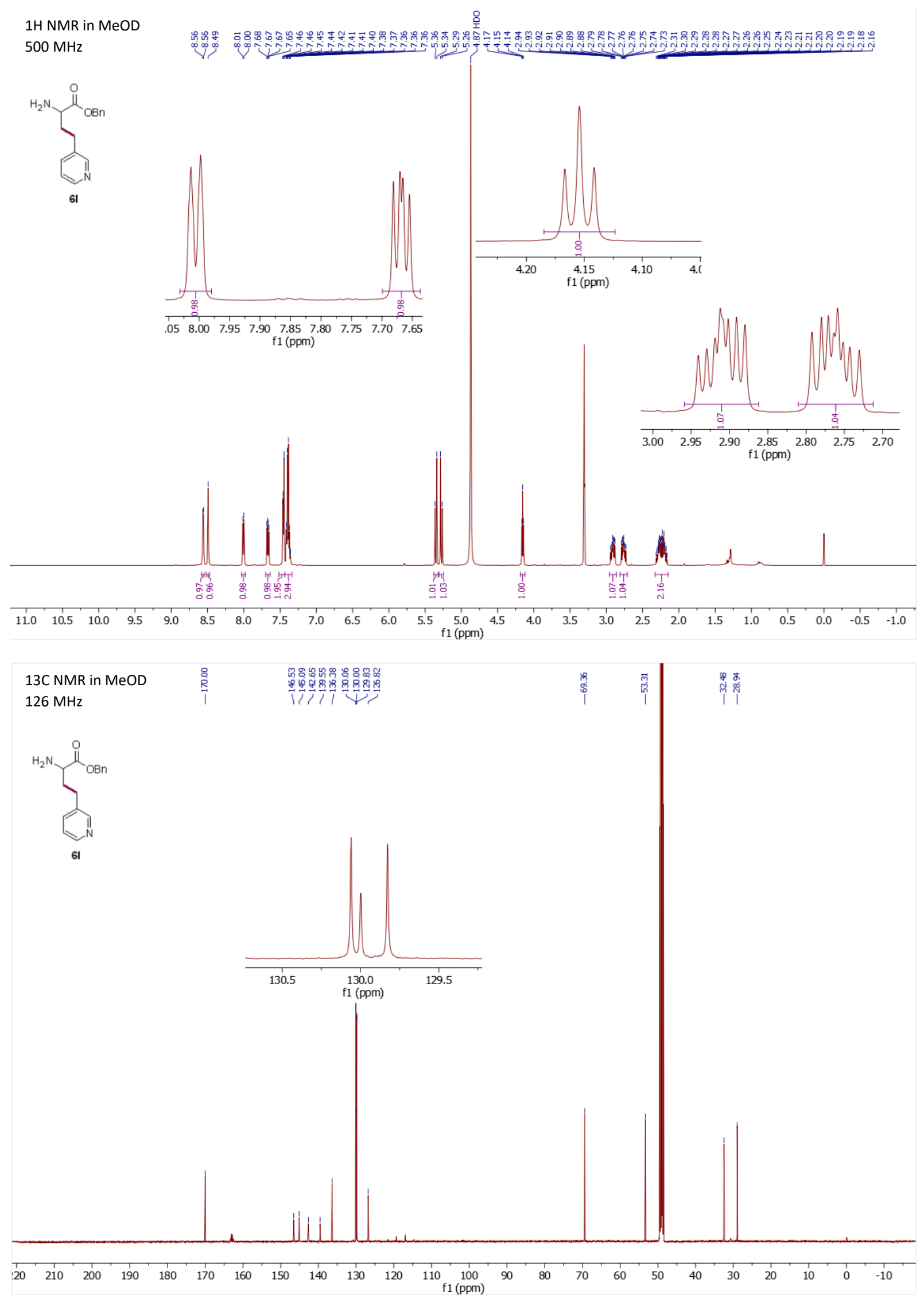




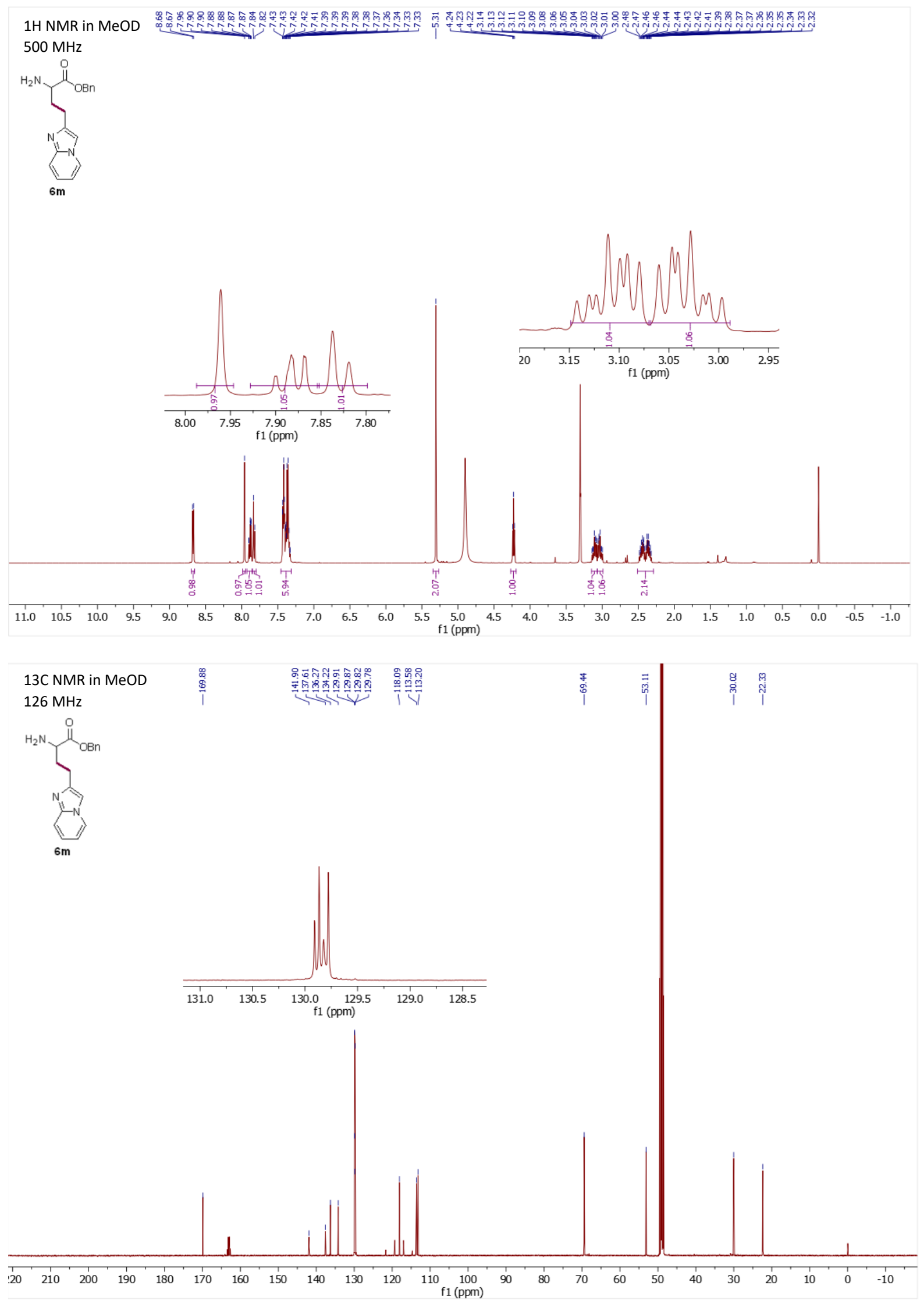



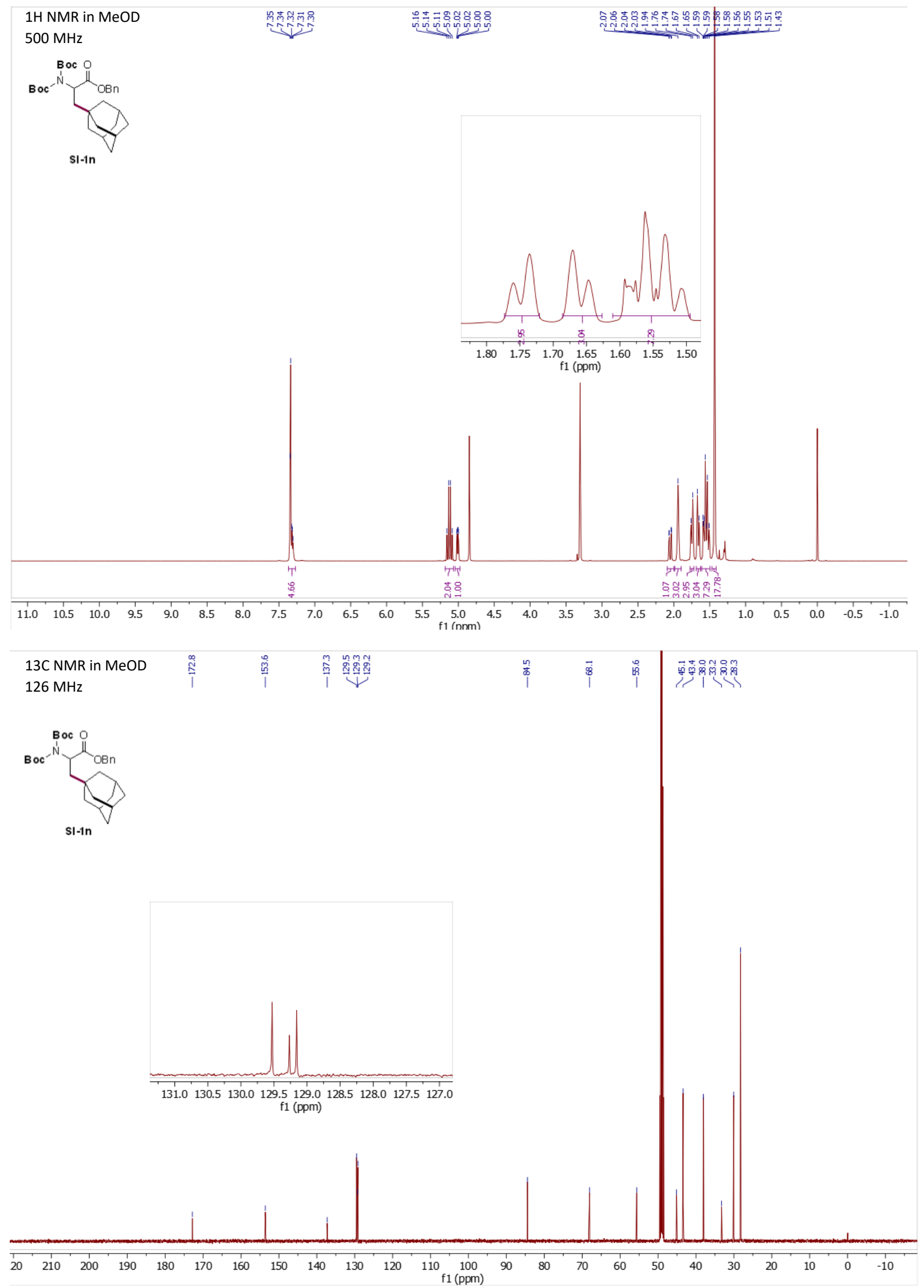

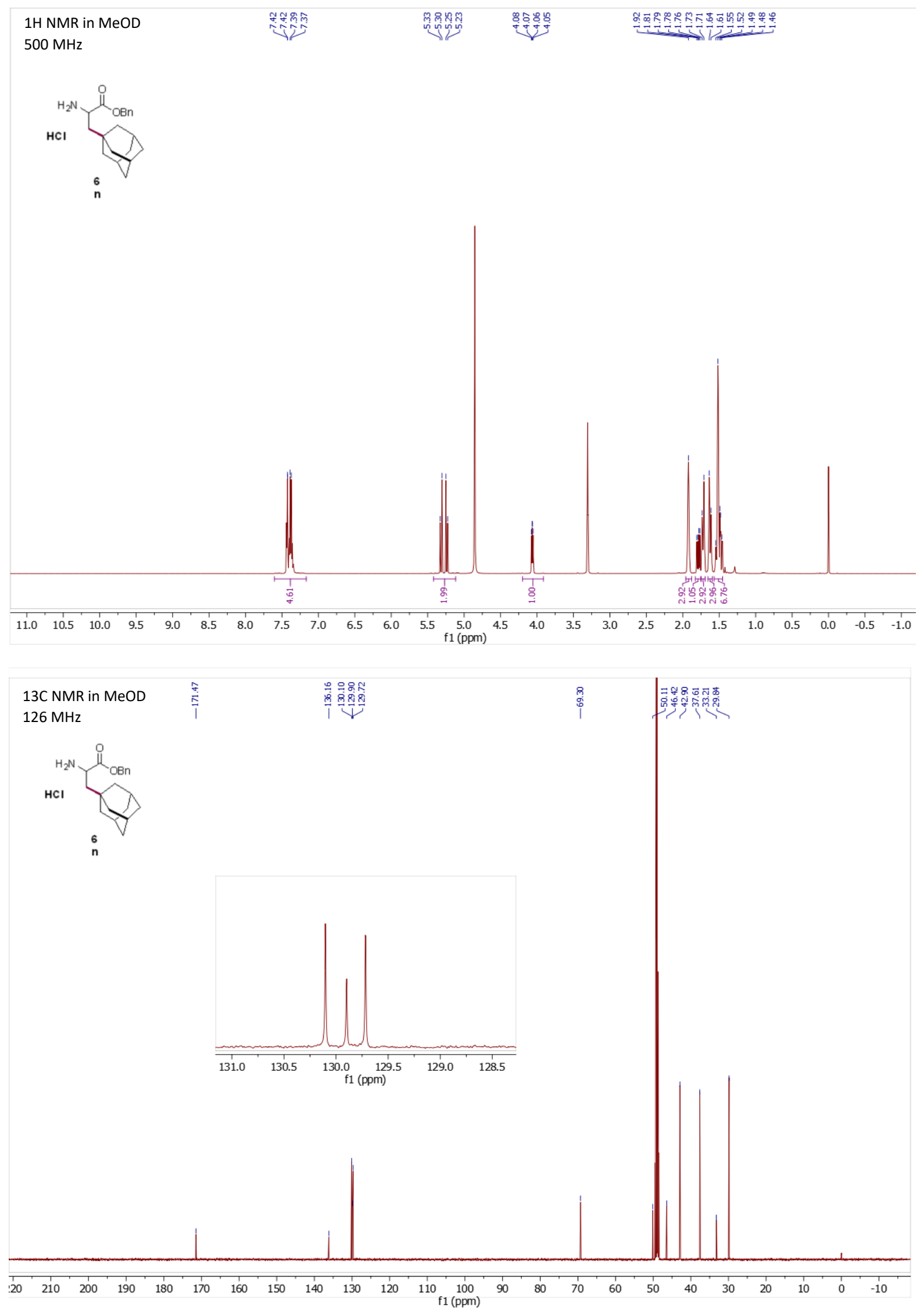

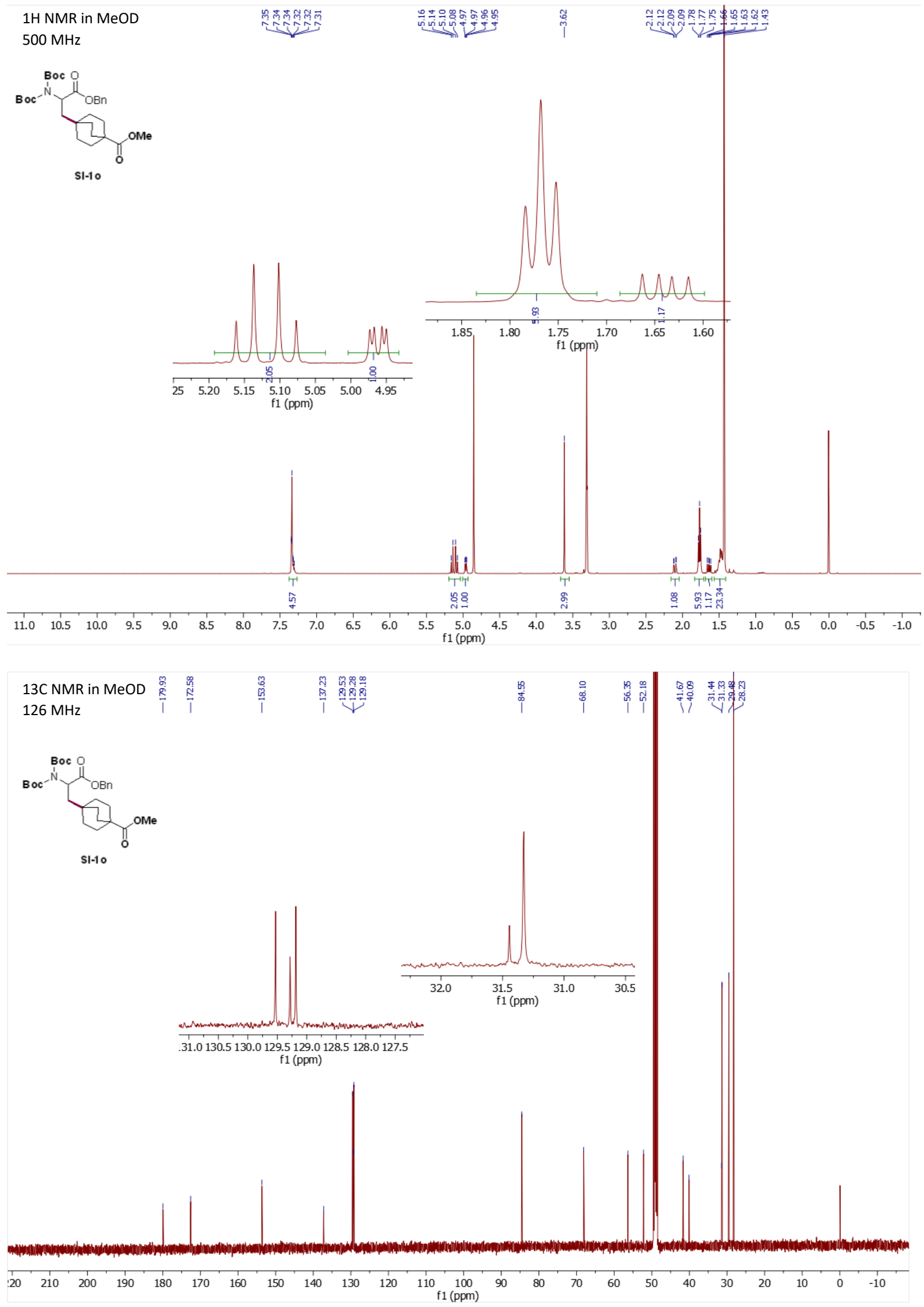


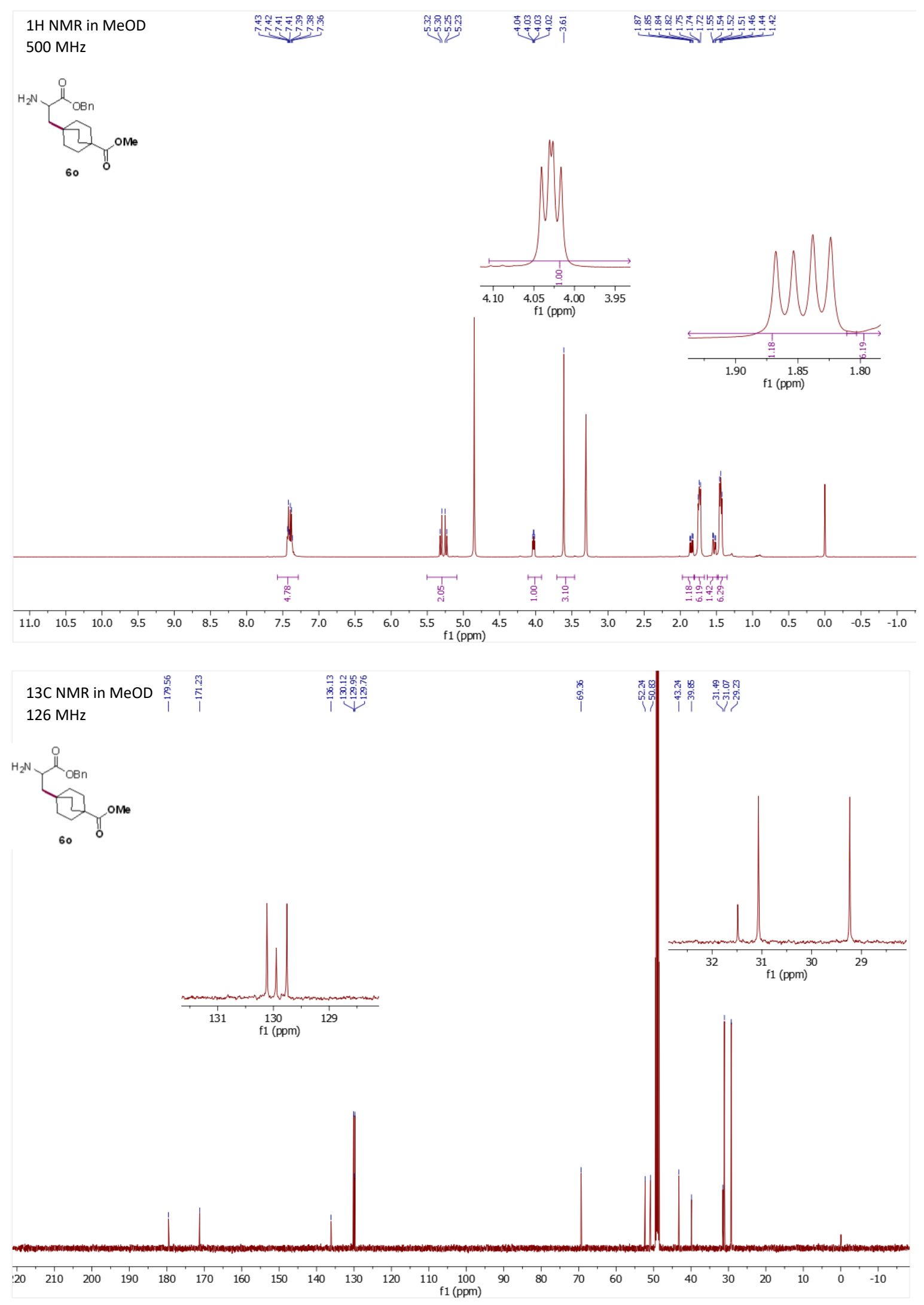




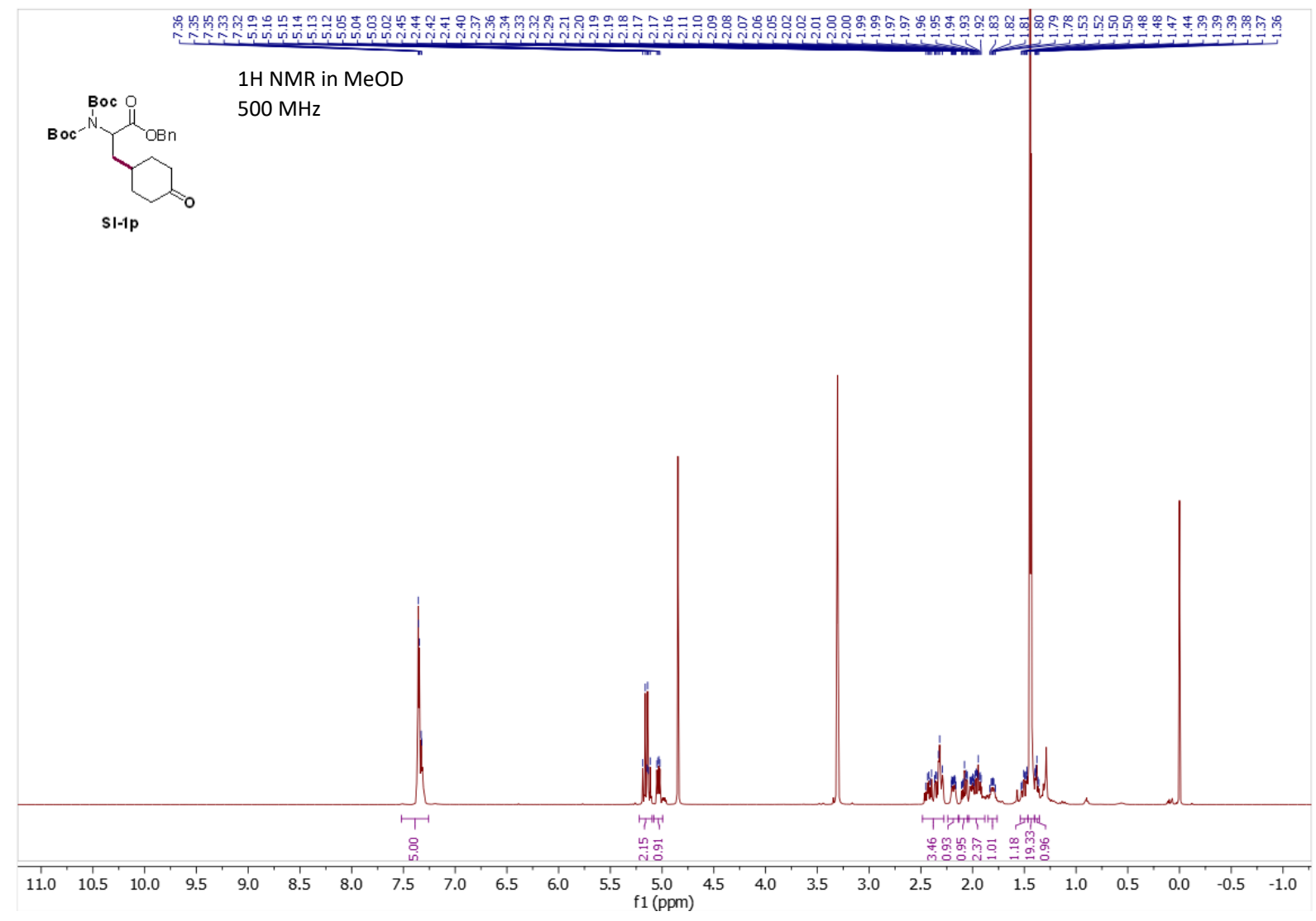

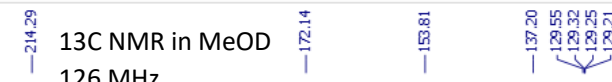
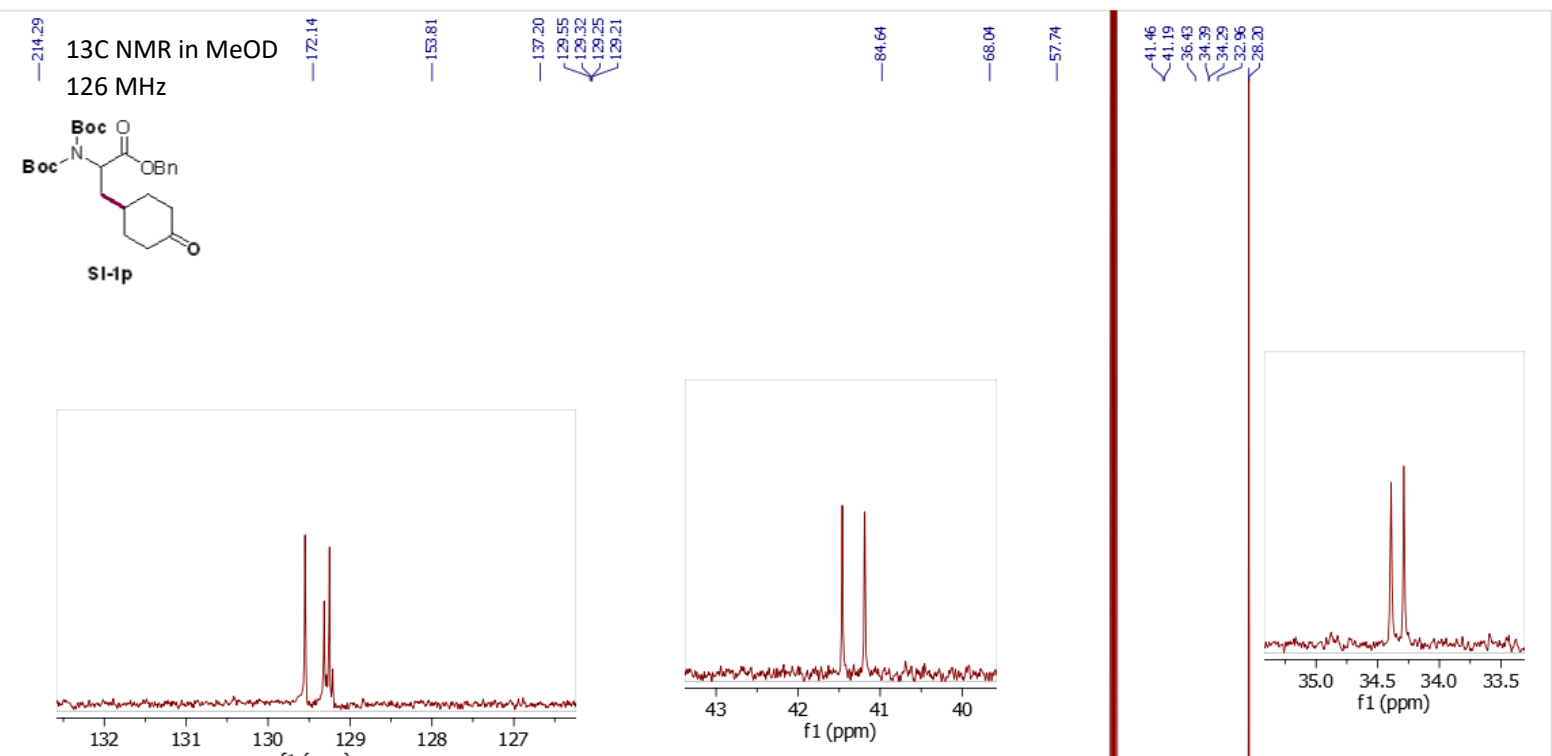

SI-1p
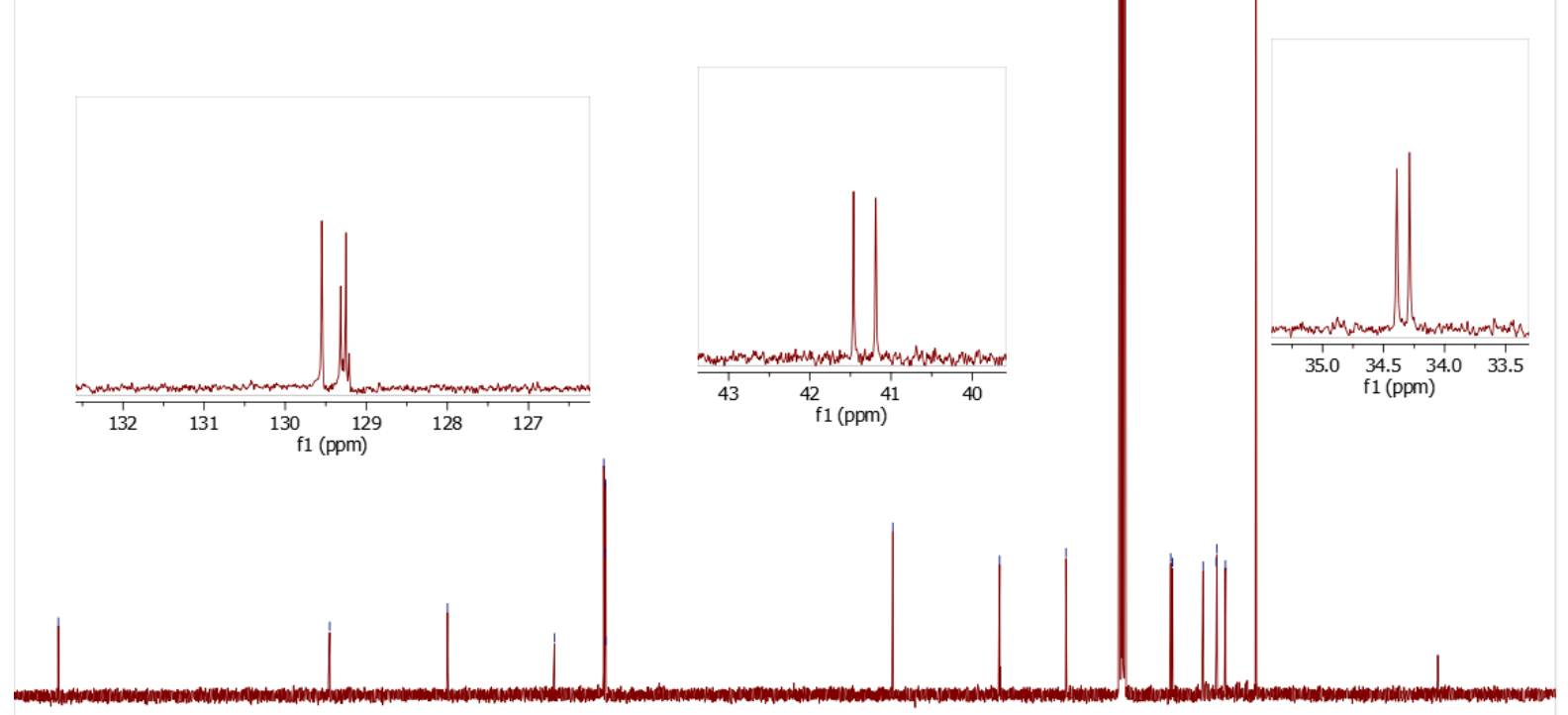

7
20

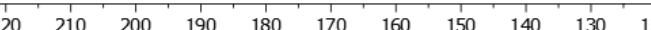

$110 \quad 100$

$1(\mathrm{ppm})$ 

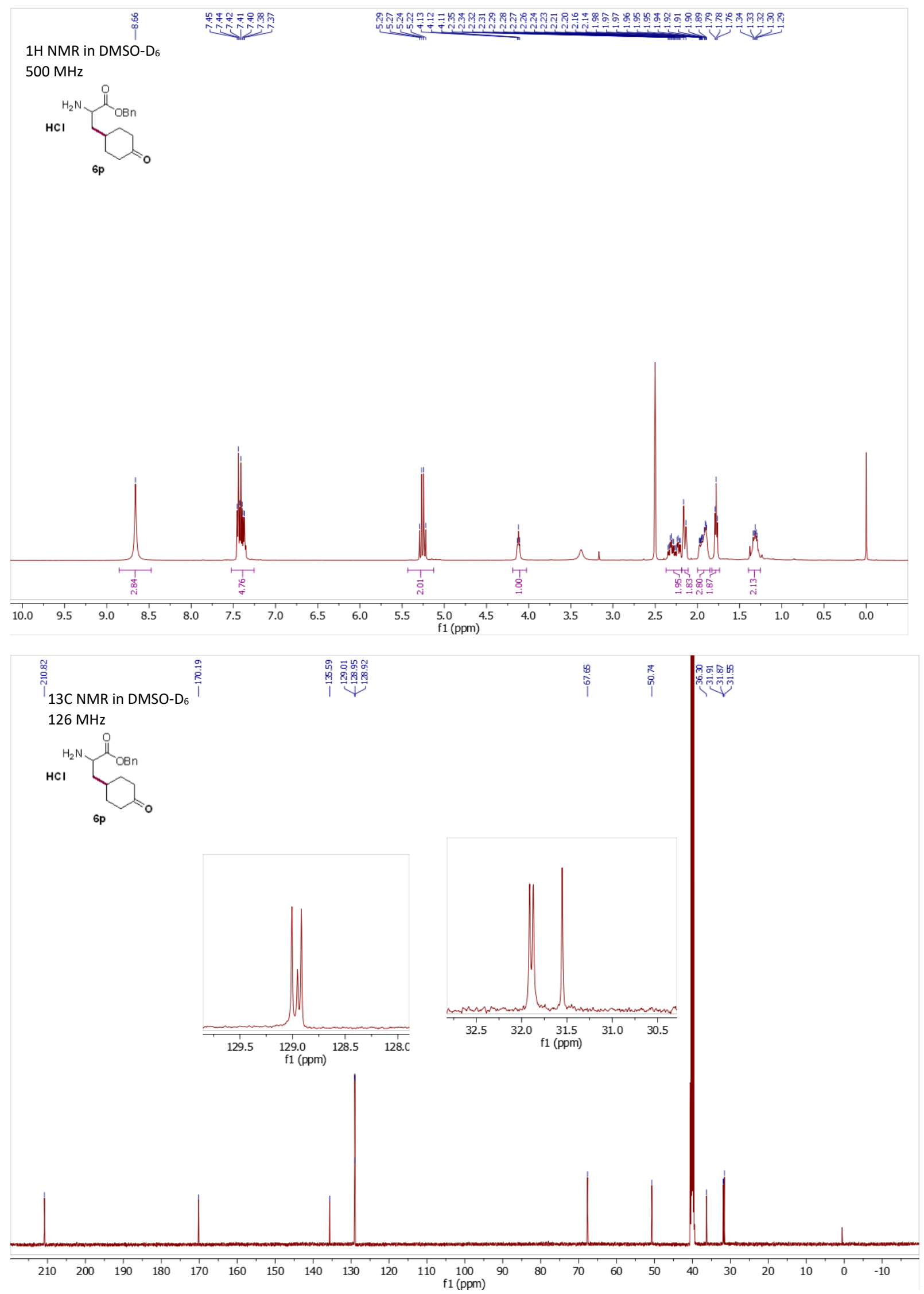

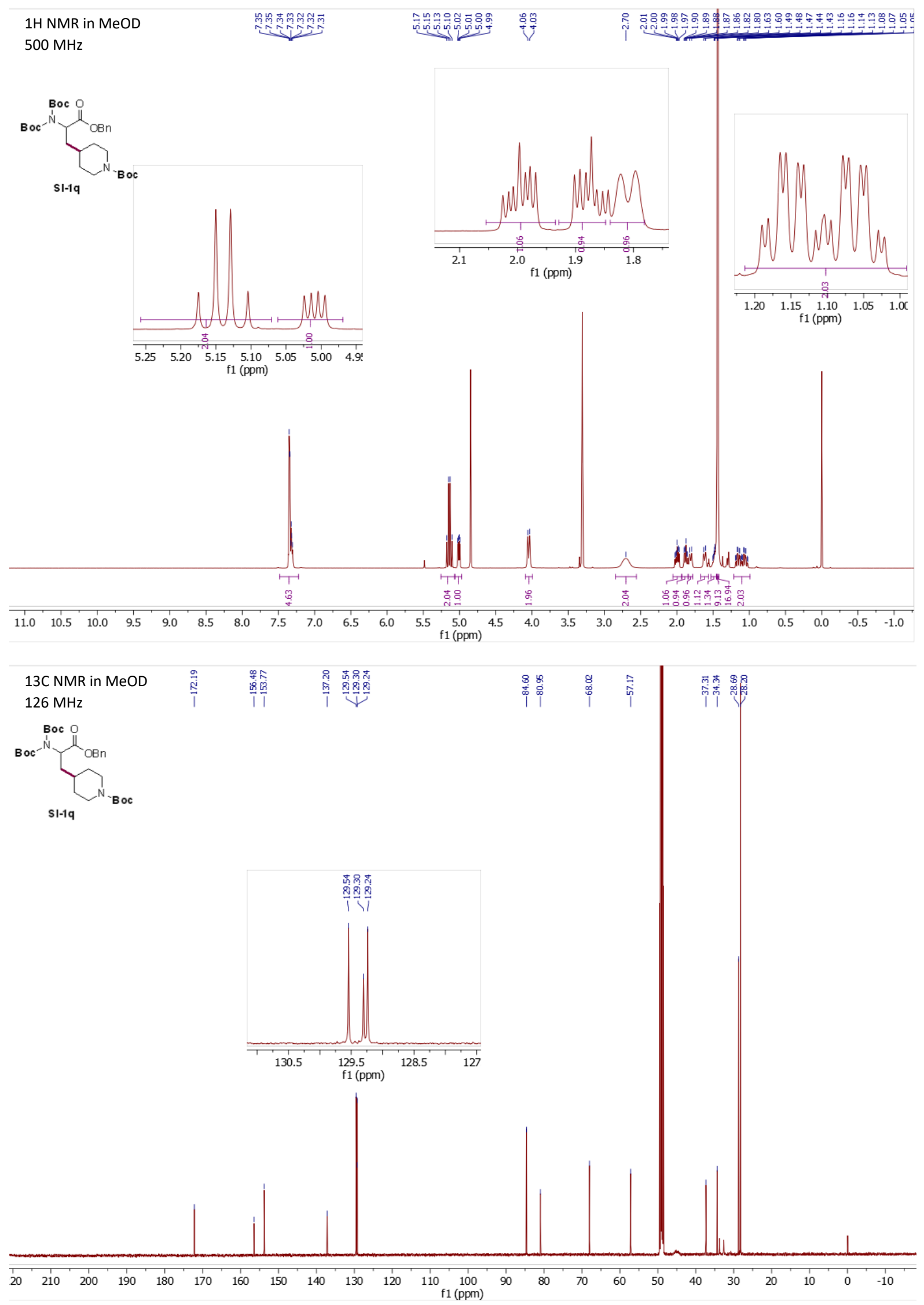

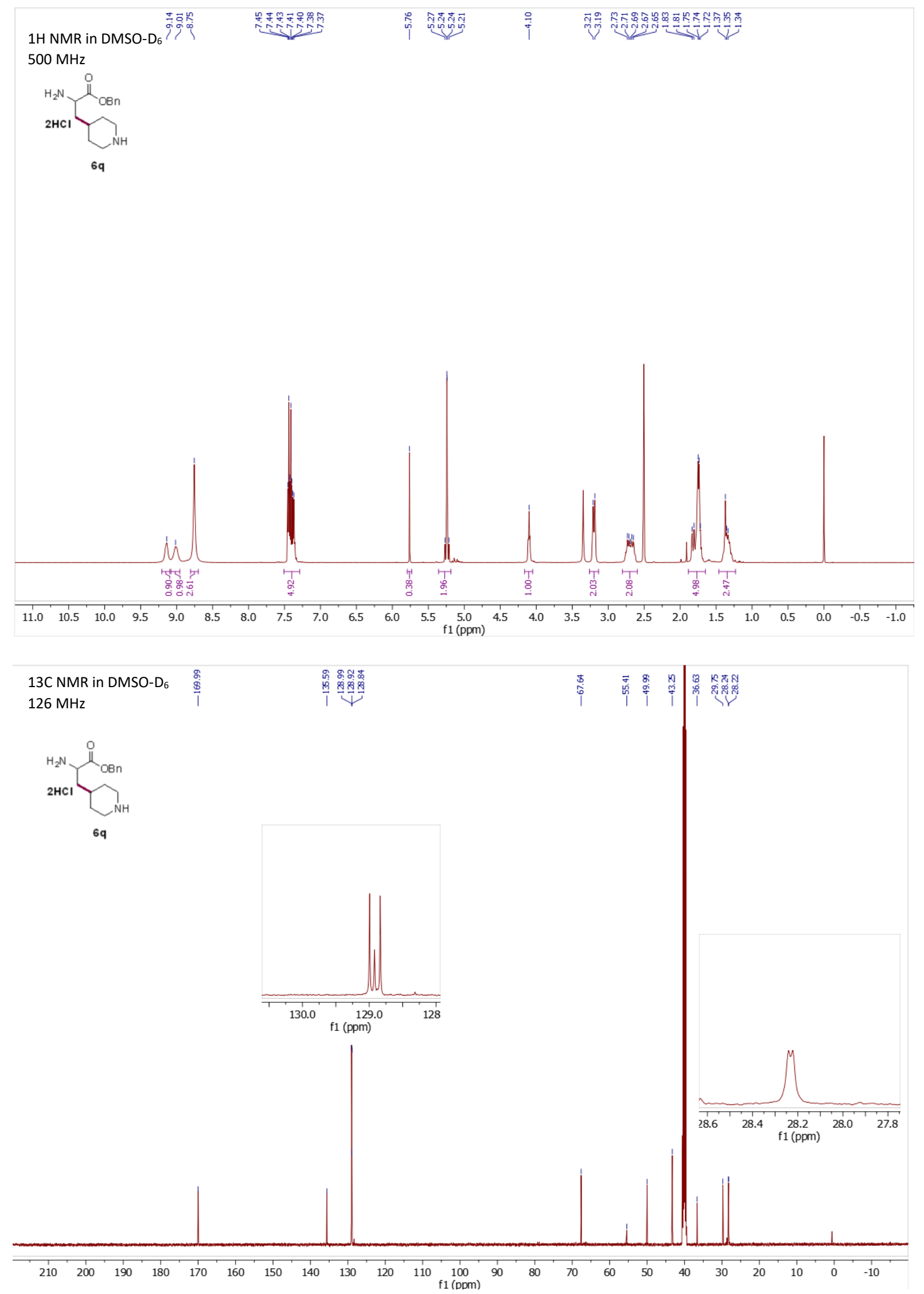


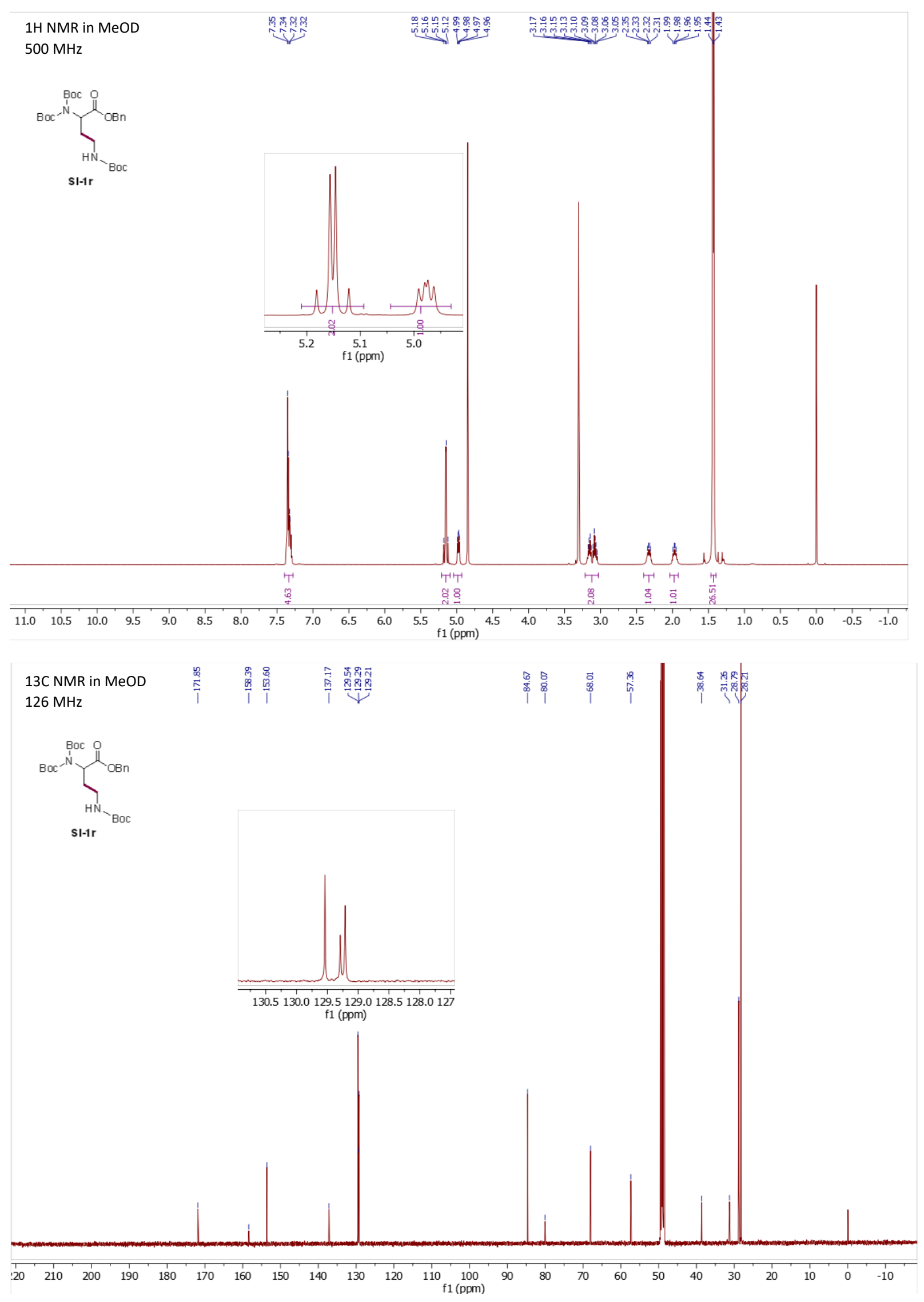



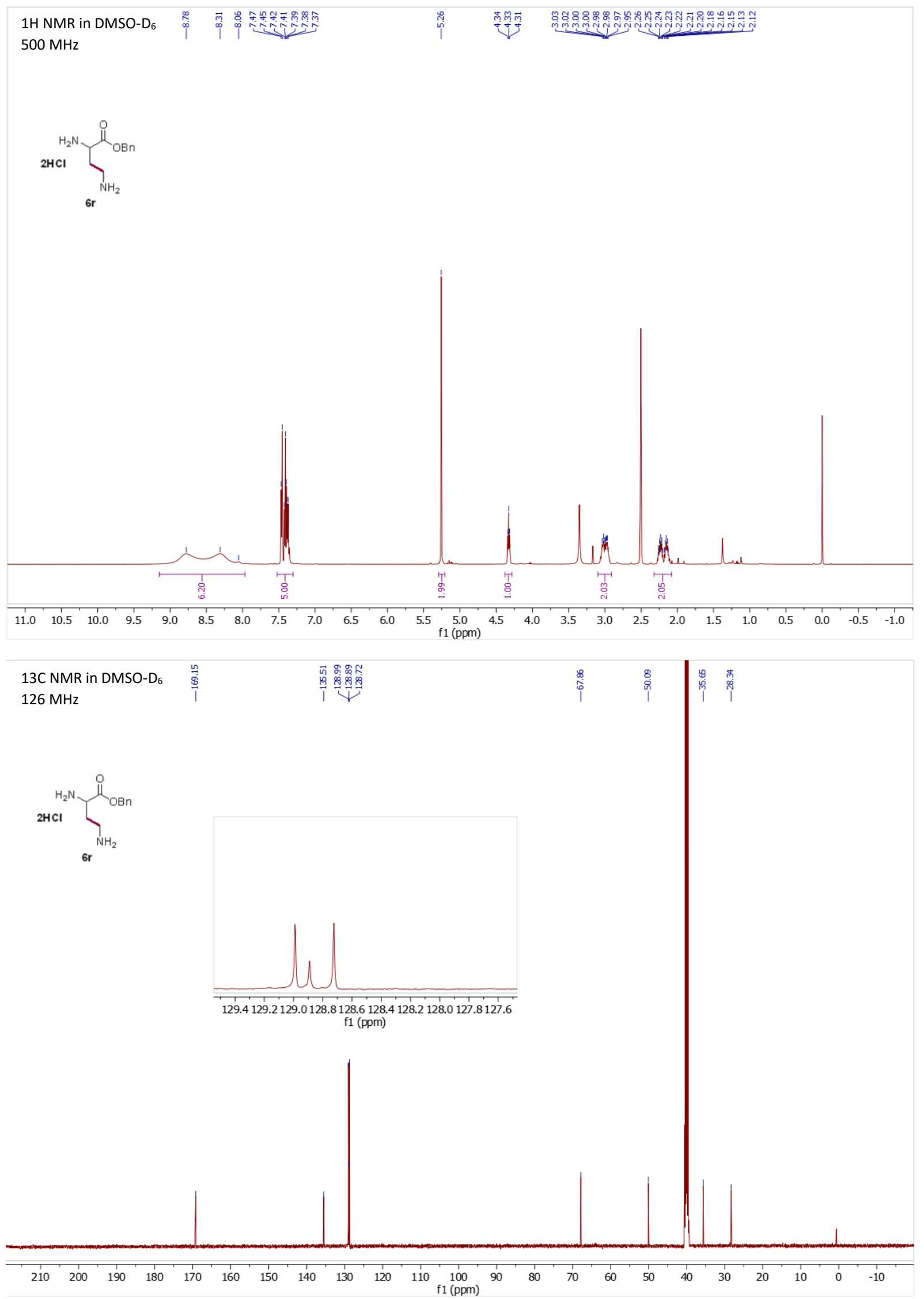

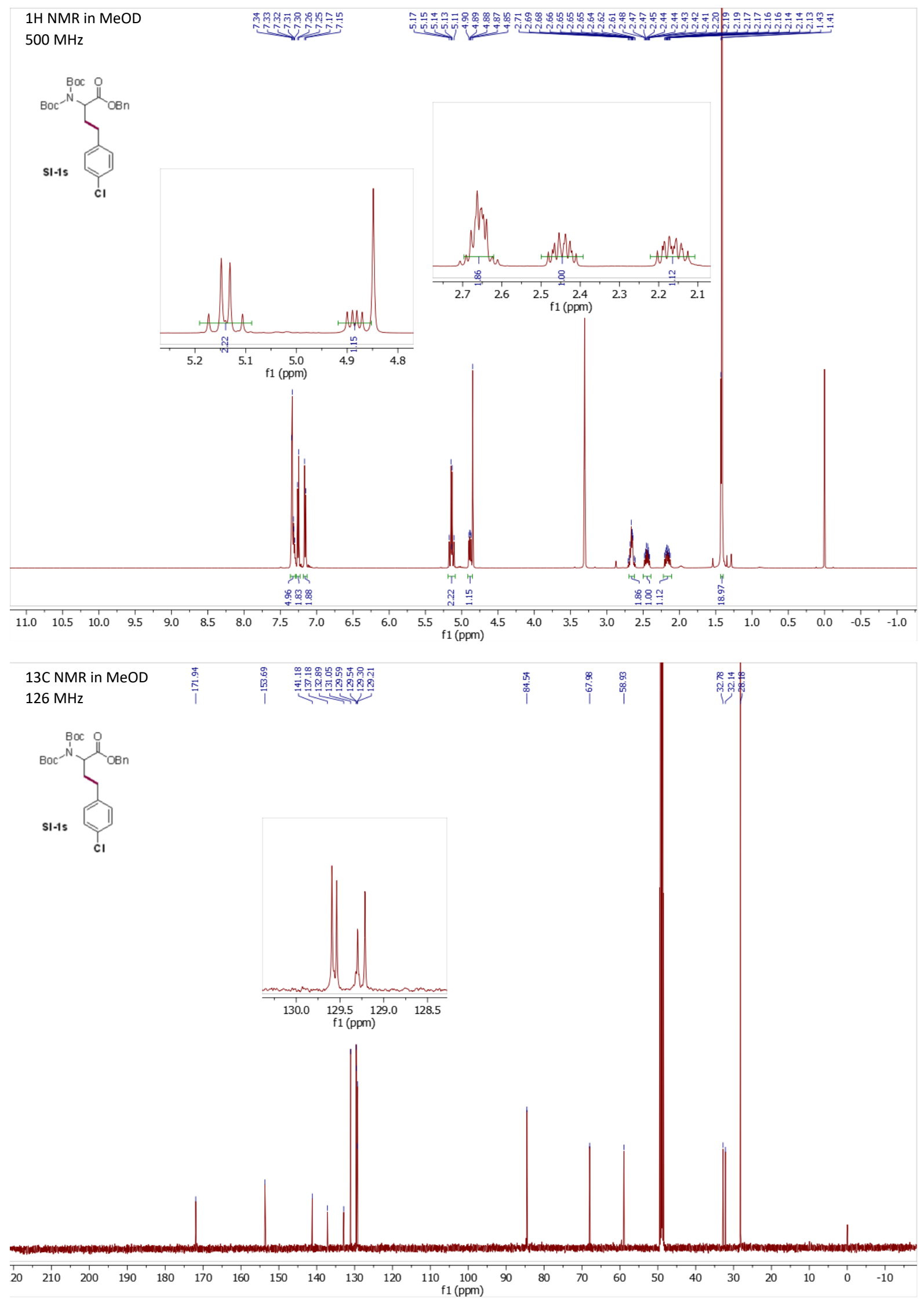

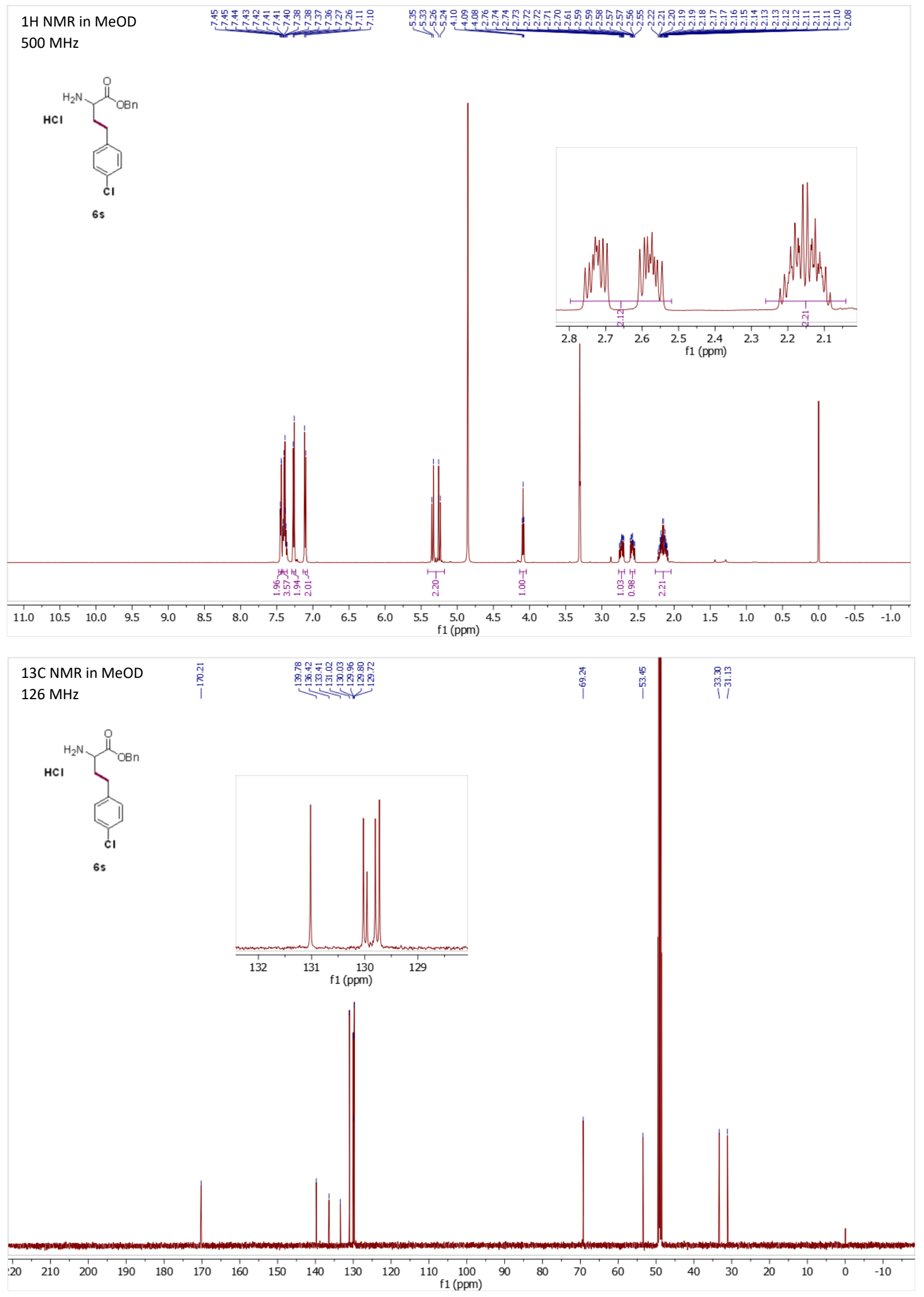

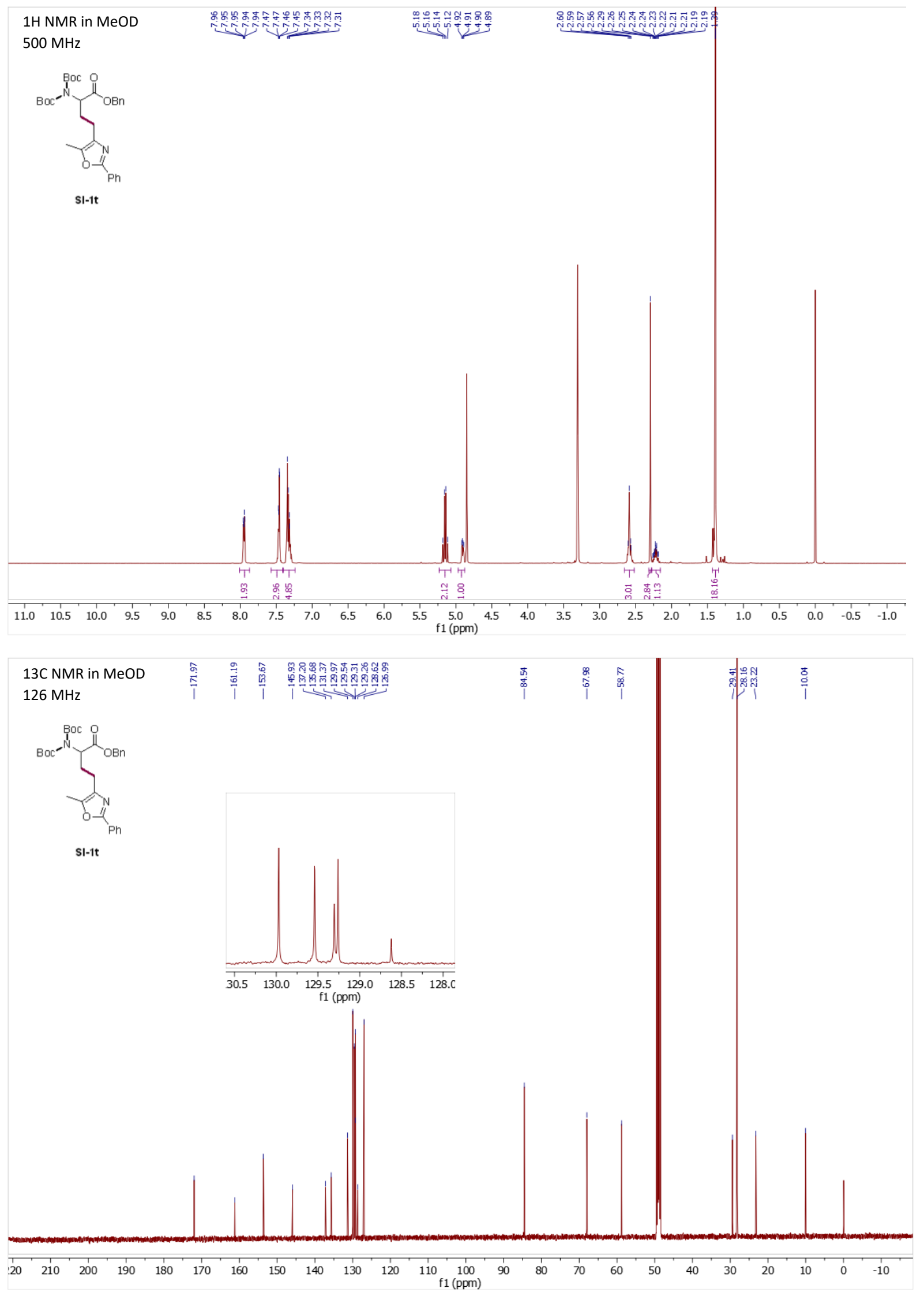

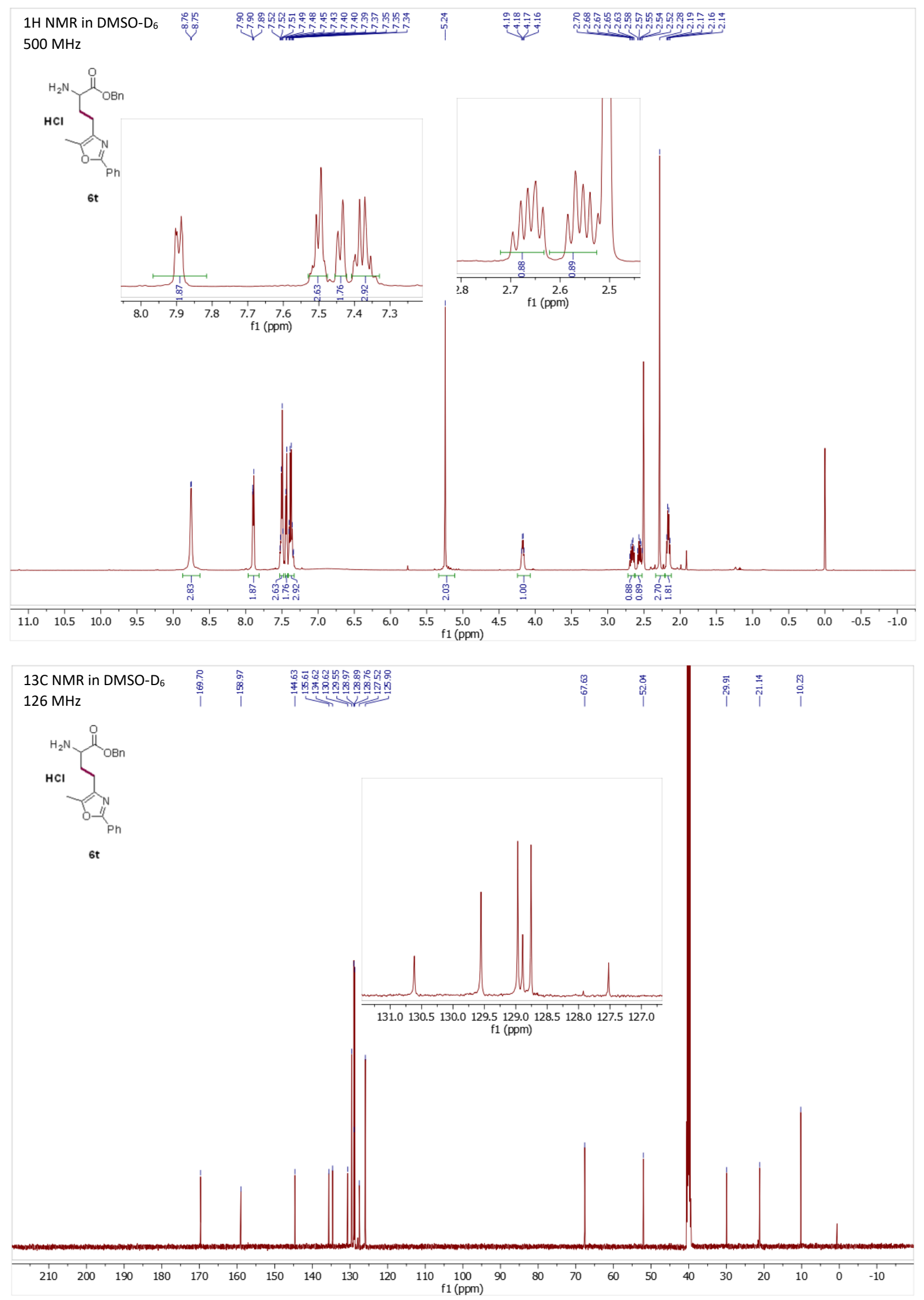

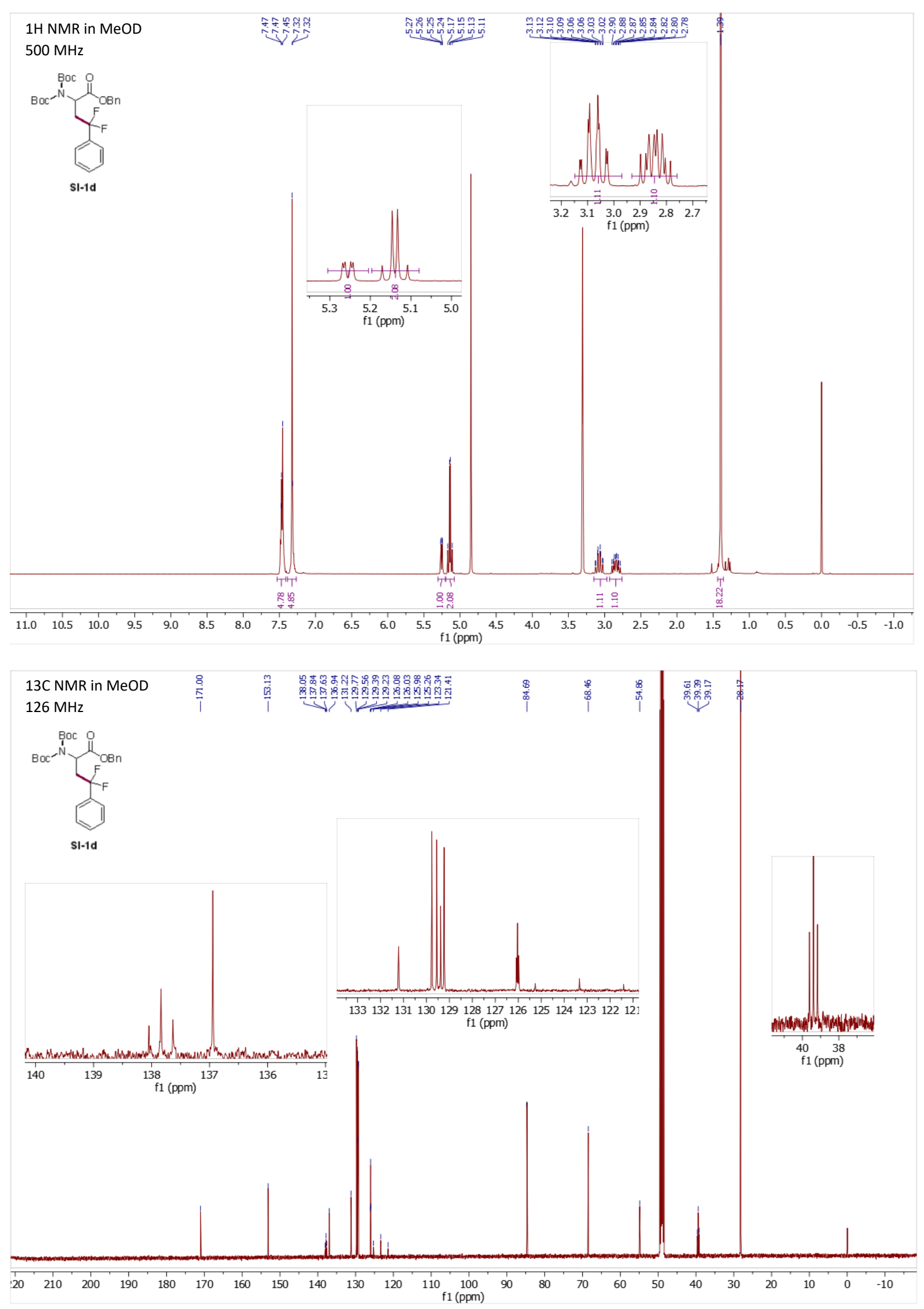

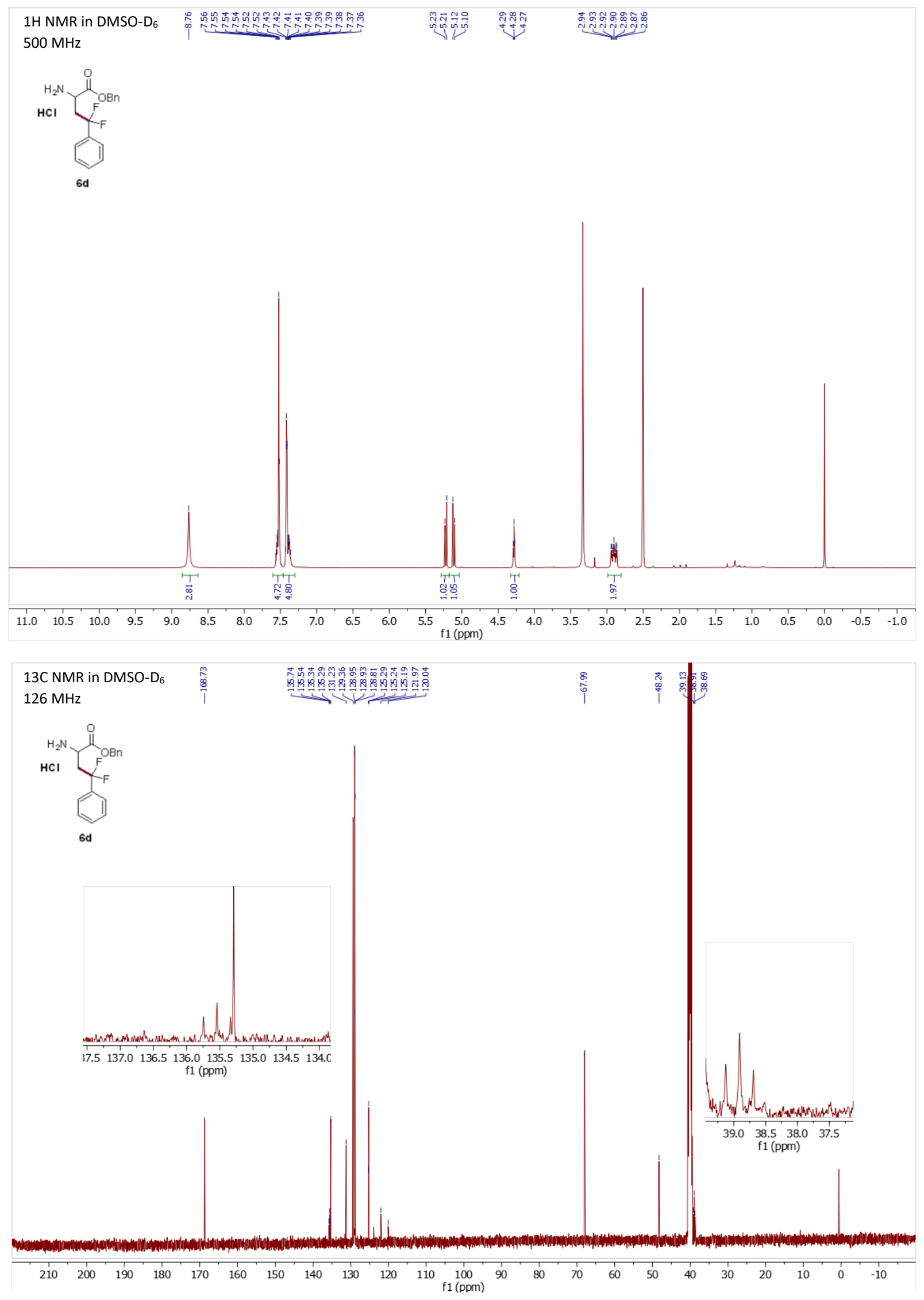

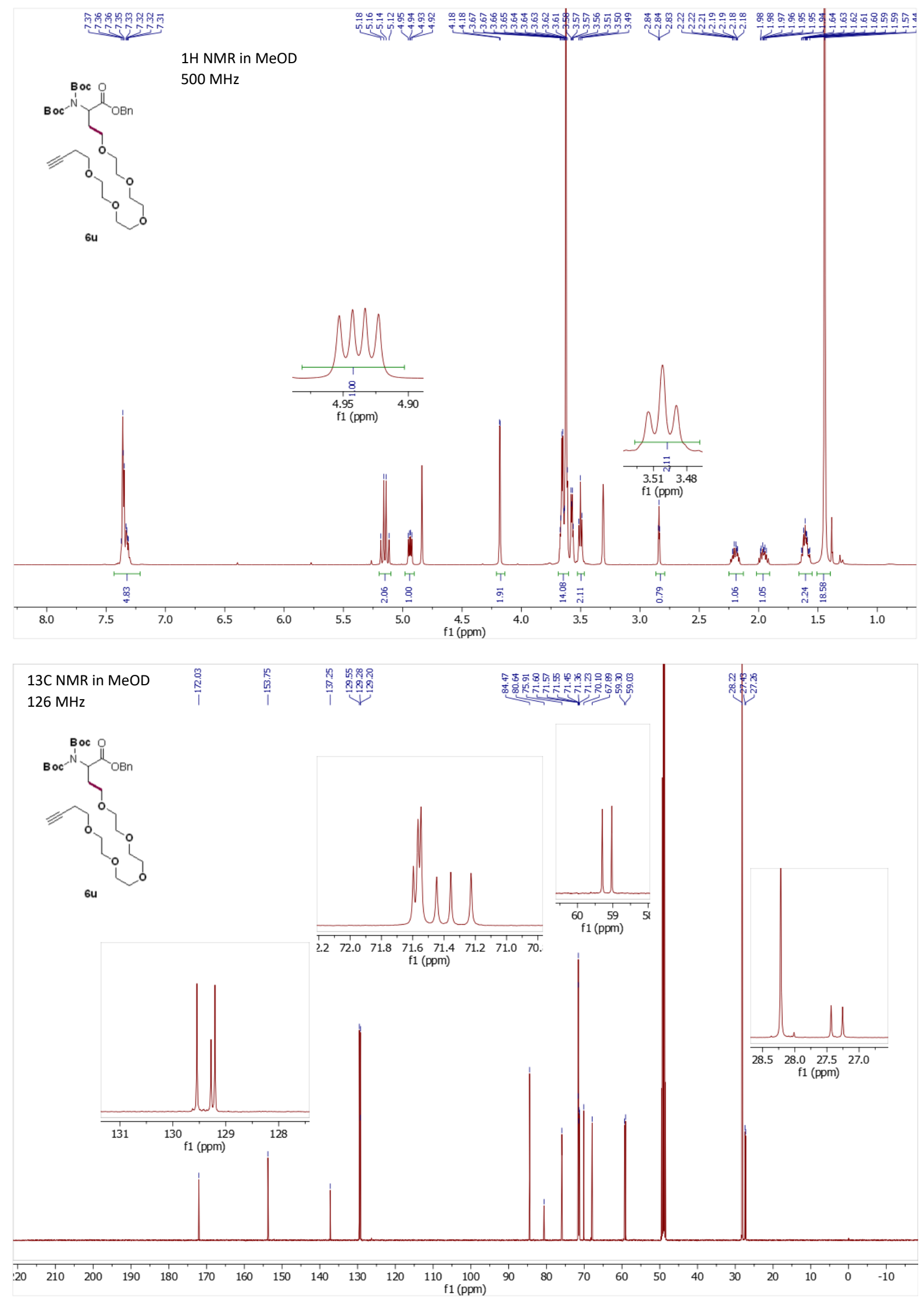

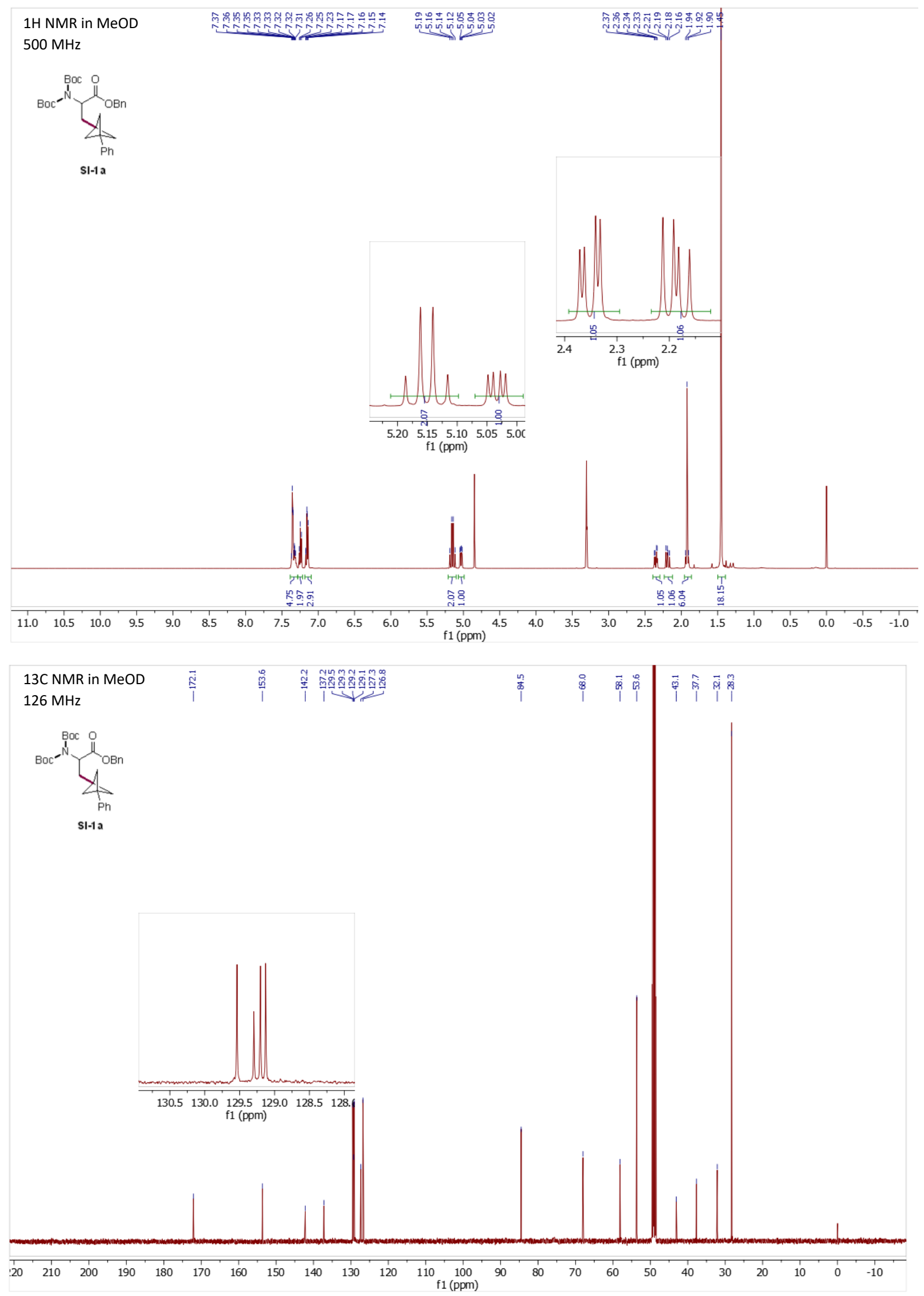